\title{
Construction of Irregular Rate-Compatible Low- Density Parity-Check Codes for Hybrid-ARQ Schemes at Short Block Lengths
}

\author{
by \\ Mohammadreza Yazdani, M. Sc.

\begin{abstract}
A thesis submitted to the
Faculty of Graduate Studies and Research

in partial fulfillment of the requirements for the degree of
\end{abstract}

\section{Master of Applied Science}

Ottawa-Carleton Institute For Electrical and Computer Engineering

Department of Systems and Computer Engineering

Faculty of Engineering

Carleton University

Ottawa, Ontario

April, 2003

(C) Copyright

Mohammadreza Yazdani, 2003 


$\begin{array}{ll}\begin{array}{l}\text { National Library } \\ \text { of Canada }\end{array} & \begin{array}{l}\text { Bibliothèque nationale } \\ \text { du Canada }\end{array} \\ \begin{array}{l}\text { Acquisitions and } \\ \text { Bibliographic Services }\end{array} & \begin{array}{l}\text { Acquisisitons et } \\ \text { services bibliographiques }\end{array} \\ \begin{array}{l}\text { 395 Wellington Street } \\ \text { Ottawa ON K1A ON4 }\end{array} & \begin{array}{l}\text { 395, rue Wellington } \\ \text { Ottawa ON K1A 0N4 } \\ \text { Canada }\end{array}\end{array}$

Your file Votre référence ISBN: 0-612-83485-9 Our file Notre référence ISBN: 0-612-83485-9

The author has granted a nonexclusive licence allowing the National Library of Canada to reproduce, loan, distribute or sell copies of this thesis in microform, paper or electronic formats.

The author retains ownership of the copyright in this thesis. Neither the thesis nor substantial extracts from it may be printed or otherwise reproduced without the author's permission.
L'auteur a accordé une licence non exclusive permettant à la Bibliothèque nationale du Canada de reproduire, prêter, distribuer ou vendre des copies de cette thèse sous la forme de microfiche/film, de reproduction sur papier ou sur format électronique.

L'auteur conserve la propriété du droit d'auteur qui protège cette thèse. $\mathrm{Ni}$ la thèse ni des extraits substantiels de celle-ci ne doivent être imprimés ou aturement reproduits sans son autorisation. 
The undersigned hereby recommend to the faculty of Graduate Studies and Research acceptance of the thesis,

\section{"Construction of Irregular Rate-Compatible Low-Density Parity-}

\section{Check Codes for Hybrid-ARQ Schemes at Short Block Lengths"}

submitted by Mohammadreza Yazdani, in partial fulfillment of the requirements for the degree of Master of Applied Science.

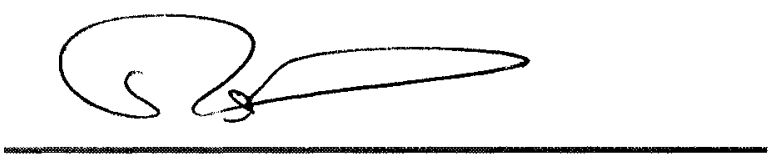

Chair, Department of Systems and Computer Engineering

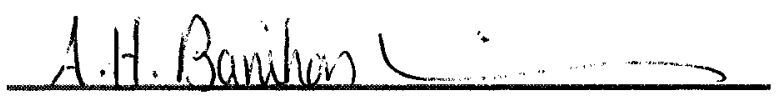

Professor Amir H. Banihashemi, Thesis Supervisor

Ottawa-Carleton Institute For Electrical and Computer Engineering Department of Systems and Computer Engineering

Faculty of Engineering

Carleton University

Ottawa, Ontario

April, 2003 


\begin{abstract}
Hybrid-ARQ protocols have the potential of providing different levels of error protection and adaptability to unknown and time-varying channels. The throughput of HybridARQ protocols is strongly influenced by the frame error rate of the rate-compatible (RC) codes used in the system. As such, designing a family of powerful RC codes with low encoding and decoding complexity is of great importance. This thesis examines different design and constructions of RC-LDPC codes at short block lengths. We first describe puncturing and extending of LDPC codes as two available techniques for generating a family of RC-LDPC codes and investigate the advantages and disadvantages of each approach. Then it is explained how a well-designed combination of these two techniques can be used for constructing a family of RC-LDPC codes with high throughput at a wide range of signal to noise ratios. We use this approach to design a family of irregular PEGconstructed RC-LDPC codes which yield a very good throughput performance while having a linear-time encoding complexity. For a data block length of 1024 , the throughput of the constructed sequence of LDPC codes can get as close as $0.7 \mathrm{~dB}$ to the channel capacity. This improves over the existing schemes based on turbo and LDPC codes by up to $0.5 \mathrm{~dB}$.
\end{abstract}




\section{Acknowledgements}

I would like to thank my thesis supervisor Professor Amir H. Banihashemi for his helpful comments and enthusiasm in my research work. He was always available for fruitful and engaged discussions regarding my work.

All my friends and colleagues at our research group and laboratory helped me a lot by providing an intellectually stimulating and enjoyable atmosphere. Without them life at Carleton would have been very difficult.

I am also grateful to Ms. Judy Bowman, our graduate assistant, for her professional help in my administrative obligations and her care and kindness. 


\section{Table of Contents}

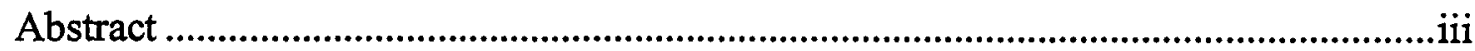

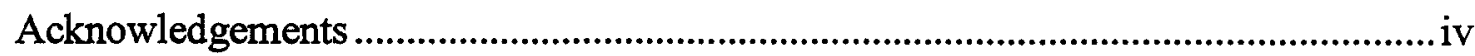

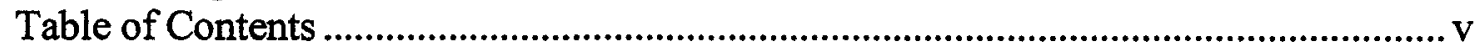

List of Figures ................................................................................................... vii

List of Abbreviations and Symbols ..............................................................................

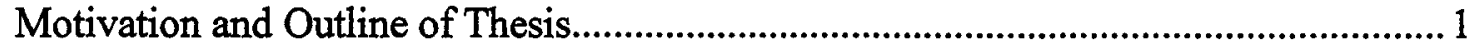

Chapter1: Low-Density Parity-Check Codes ................................................................. 3

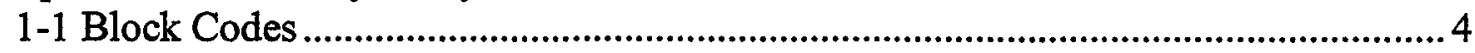

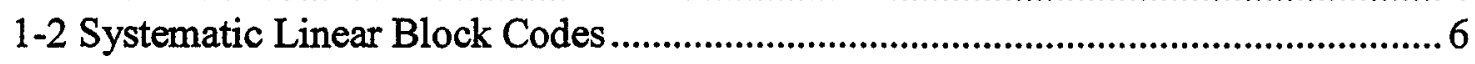

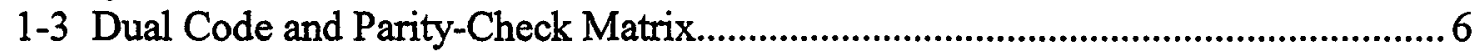

1-4 Minimum Distance of a Block Code .............................................................. 8

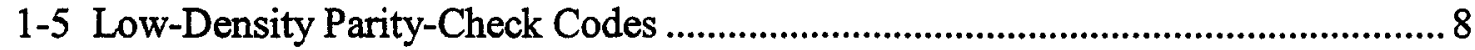

1-6 Comparison of LDPC Codes and Turbo Codes.................................................. 10

Chapter 2: Constructing, Encoding, and Decoding of LDPC Codes ............................. 12

2-1 Constructing and Encoding of LDPC Codes .................................................. 13

2-1-1 Some Definitions and Notations............................................................. 13

2-1-2 Different Constructions of LDPC Codes................................................ 17

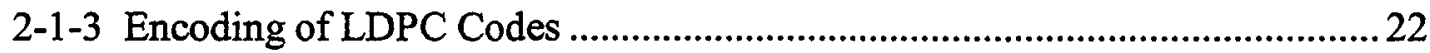

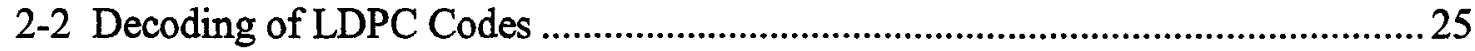

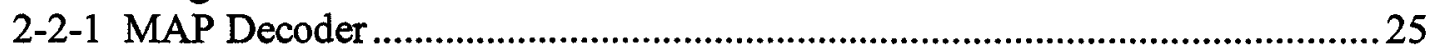

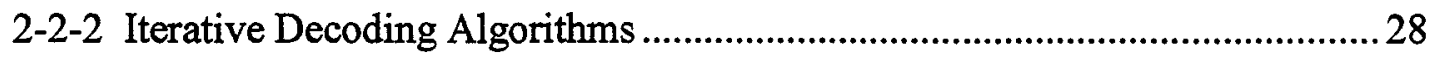

2-2-2-1 Belief Propagation Algorithm..................................... 31

2-3 Performance of LDPC Codes under Belief-Propagation Algorithm ..................... 33

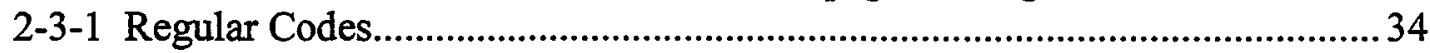

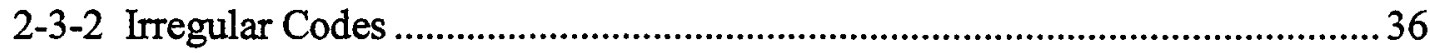

2-3-3 Linear-Time Encodable Irregular LDPC Codes......................................... 40

Chapter 3: Automatic Repeat Request Protocls ................................................... 42

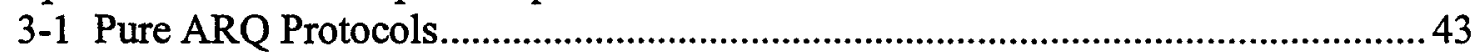

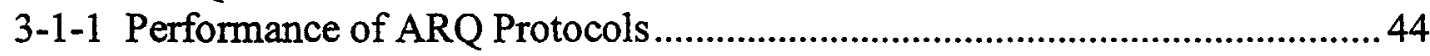

3-1-1-1 Reliability.....................................................44

3-1-1-2 Throughput...................................................45

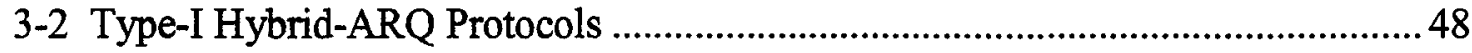

3-3 Type-II Hybrid-ARQ Protocols ....................................................................... 49

Chapter 4: A Type-II Hybrid-ARQ Protocol Based on PEG-Constructed Rate-Compatible

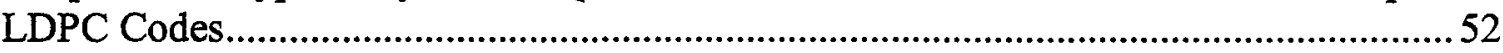

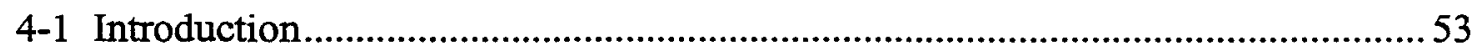

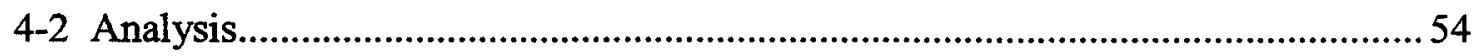

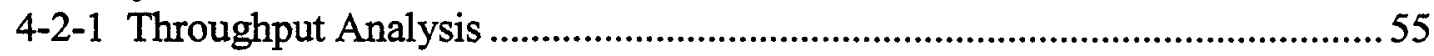

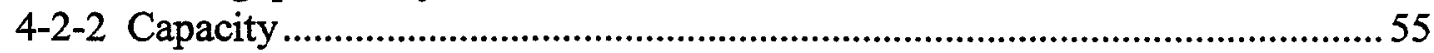

4-3 Type-II Hybrid-ARQ Protocols Using RCPT Codes............................................58

4-4 Type-II Hybrid-ARQ Protocols Based on RC-LDPC Codes ................................60

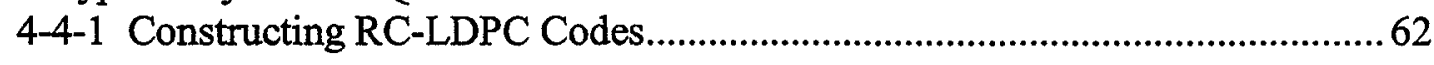




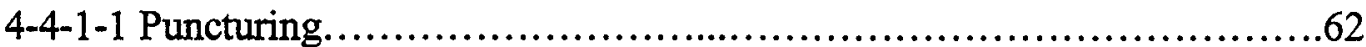

4-4-1-2 Extending... ..................................................65

4-4-2 Construction of an Efficient Family of RC-LDPC Codes........................... 73

4-4-3 Performance of Type-II Hybrid-ARQ Protocol Based on PEG-RC-LDPC

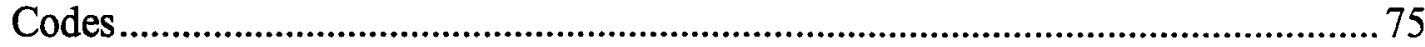

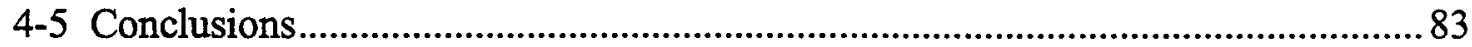

4-6 Ideas for Future Research Work and Outlook .................................................... 83

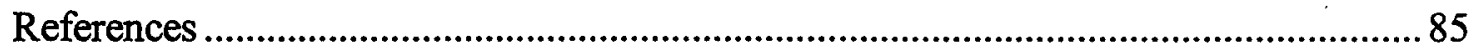




\section{List of Figures}

Figure 1.1. Tanner graph of the (3,5)-regular LDPC

Figure 2.1. An irregular Tanner graph with $D_{s}=\{2,2,2,2,3,3,3,4\}$ and $D_{c}=\{4,4,5,6\}$

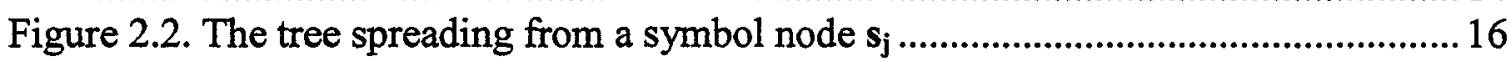

Figure 2.3. The tree spreading from a check node $\mathbf{c}_{\mathbf{i}}$.....................................................16

Figure 2.4. Parity-check matrix of a (3, 4)-regular LDPC code with $n=20$ and $m=15$..... 18

Figure 2.5. Decision region of the MAP decoding for a priori probabilities $3 / 16,5 / 16$, $3 / 16$ and 5/16 (a) and 4/16, 4/16,4/16, and 4/16 (b). Note that (b) also represents ML decoding. .....................................................................................................27

Figure 2.6. Before going through iterations, initial messages are sent to check nodes.....30

Figure 2.7. (a) Check nodes $c$ sending a new message to symbol node $s_{d_{c}}$. (b) Symbol node $\mathrm{s}$ sending back updated message to check node $\mathrm{c}_{\mathrm{d}_{\mathrm{s}}}$

Figure 2.8. BER and WER of a PEG-constructed code and MacKay's code with parameters $n=504, m=252, d_{s}=3, d_{c}=6$. .35

Figure 2.9. BER and WER of a PEG Tanner-graph code and MacKay's code with parameters $n=1008, m=504, d_{s}=3, d_{c}=6$.

Figure 2.10. BER and WER of an irregular PEG Tanner-graph, irregular random-graph code, and MacKay's regular code with parameters $n=504, m=252$.

Figure 2.11. BER and WER of an irregular PEG Tanner-graph, irregular random-graph code, and MacKay's regular code with parameters $n=1008, m=504$.

Figure 2.12. BER and WER of an irregular PEG Tanner-graph and an irregular upper-

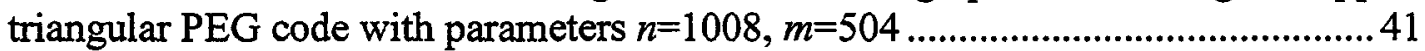

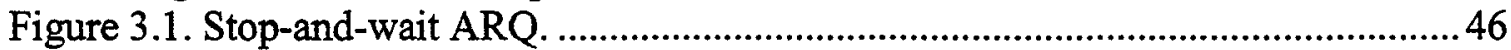

Figure 3.2. Go-back-N with $\mathrm{N}=5$. ......................................................................... 47

Figure 3.3. Selective-repeat ARQ ................................................................................... 48

Figure 4.1. Capacity of a binary-input, AWGN channel. ............................................58

Figure 4.2. Throughput of type-II hybrid-ARQ system using RCPT codes, from [45] ....60

Figure 4.3. Performance of codes obtained by puncturing a (3, 9)-regular LDPC code of rate-16/24 with $k=2048$. Solid lines: Performance of RC codes obtained by puncturing. Dashed lines: Performance of typical codes with the same block lengths and rates as punctured codes. 64

Figure 4.4. Performance of codes obtained by puncturing an irregular LDPC code of rate$8 / 14$ with $k=1024$. Solid lines: Performance of RC codes obtained by puncturing. Dashed lines: Performance of typical codes with the same block lengths and rates as punctured codes

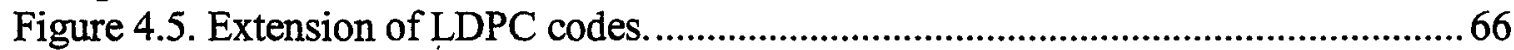

Figure 4.6. Illustration of the extension of the rate-4/8, $(3,6)$-regular LDPC code with $k=1024$

Figure 4.7. Performance of codes obtained by extending a regular- $(3,6)$ LDPC code of rate-4/8 with $k=1024$ Solid lines: Performance of RC codes obtained by extending. Dashed lines: Performance of typical codes of the same block lengths and rates as extended codes.. 
Figure 4.8. Illustration of the extension of the rate-8/14, irregular LDPC code with $k=1024$

Figure 4.9. Performance of codes obtained by extending an irregular LDPC code of rate8/14 with $k=1024$. Solid lines: Performance of $\mathrm{RC}$ codes obtained by extending. Dashed lines: Performance of typical codes of the same block lengths and rates as extended codes.

Figure 4.10 Comparing the performance of the rate-8/16 code of Fig.4.9, obtained by extending, with a similar rate- $8 / 16$ code, obtained by Poisson construction..

Figure 4.11. Throughput performance of the type-II hybrid-ARQ protocol based on the family of irregular RC-LDPC codes with $k=1024$, constructed in Example 4.1, and the throughput obtained by regular RC-LDPC codes ([49]) and RCPT codes ([45] ).

Figure 4.12. Illustration of the extension of the rate-8/13, PEG-RC-LDPC code with $k=1024$, and the symbol- degree distribution of equation (4.12). 74

Figure 4.13. Performance of codes obtained by puncturing a PEG-RC-LDPC code of rate-8/13 with $\mathrm{k}=1024$ and with symbol node degree distribution given by equation (4.12). Solid lines: Performance of RC codes obtained by puncturing. Dashed lines: Performance of typical codes with the same block lengths and rates as the corre sponding punctured codes.

Figure 4.14. Performance of codes obtained by extending a PEG-RC-LDPC code of rate$8 / 13$ with $k=1024$ and with symbol node degree distribution given by equation (4.12). Solid lines: Performance of RC codes obtained by extending. Dashed lines: Performance of typical codes with the same block lengths and rates as the corresponding extended codes 81

Figure 4.15. Evaluating the performance of the rate $8 / 14$ and the rate-8/15 codes of Fig. 4.14, obtained by extending. Solid lines: Performance of RC codes obtained by extending. Dot-Dashed lines: Performance of the codes with the same block lengths, rates and degree distributions of as the corresponding extended codes. Dashed lines: Performance of typical codes with the same block lengths and rates as the corresponding extended codes. 81

Figure 4.16. Throughput performance of type-II hybrid-ARQ protocol based on the family of PEG-RC-LDPC codes with $k=1024$ and the throughput obtained by similar regular RC-LDPC codes ([49]) and RCPT codes ([45]). 82

Figure 4.17 Throughput performance of type-II hybrid-ARQ protocol based on the family of PEG-RC-LDPC codes with $k=1024$ in comparison with the upper bound for throughput of PEG-RC-LDPC codes at this data block length 82 


\section{List of Abbreviations and Symbols}

\begin{tabular}{|c|c|}
\hline$A C K$ & (positive) Acknowledgment \\
\hline$A P P$ & A Posteriori Probability \\
\hline$A R Q$ & Automatic Repeat Request \\
\hline$B E R$ & Bit Error Rate \\
\hline$B L A W G N$ & Binary Input Additive White Gaussian Noise \\
\hline$B P$ & Belief Propagation \\
\hline$C R C$ & Cyclic Redundancy Check \\
\hline$F E C$ & Forward Error Correction \\
\hline$L D P C$ & Low-Density Parity-Check \\
\hline$L L R$ & Log-Likelihood Ratio \\
\hline$M A P$ & Maximum A Posteriori \\
\hline$M L$ & Maximum-Likelihood \\
\hline$N A K$ & Negative Acknowledgment \\
\hline$P E G$ & Progressive-Edge Growth \\
\hline PEG-RC-LDPC & Linear-time encodable PEG-constructed RC-LDPC codes \\
\hline$R C$ & Rate-Compatible \\
\hline$R C P T$ & Rate-Compatible Punctured Turbo \\
\hline SISO & Soft-Input, Soft-Output \\
\hline$W E R$ & Word Error Rate \\
\hline
\end{tabular}




\section{Sumbols}

A code alphabet

C a block code

$n$

length of a block code

$q$

alphabet size of a block code

$M \quad$ number of codewords in a block code

$R \quad$ rate of a block code

g

$i$ th row of the generator matrix

m

a message block

$k$

length of a message block

$m_{i}$

$i$ th element of a message block

c

a codeword of a block code

$G$

generator matrix of a linear block code

H

parity-check matrix of a linear block code

$\boldsymbol{h}_{i}$

$i$ th row of parity-check matrix

$C^{1}$

dual code of a linear block code $C$

$d\left(c_{1}, c_{2}\right) \quad$ Hamming distance between two codewords $c_{1}$ and $c_{2}$

$d_{\min } \quad$ minimum distance of a block code

$d_{s} \quad$ symbol-degree of a regular LDPC code

$d_{c} \quad$ check-degree of a regular LDPC code

$m \quad$ number of rows of parity-check matrix

$c_{i} \quad i$ th check node

$s_{j} \quad j$ th symbol node 


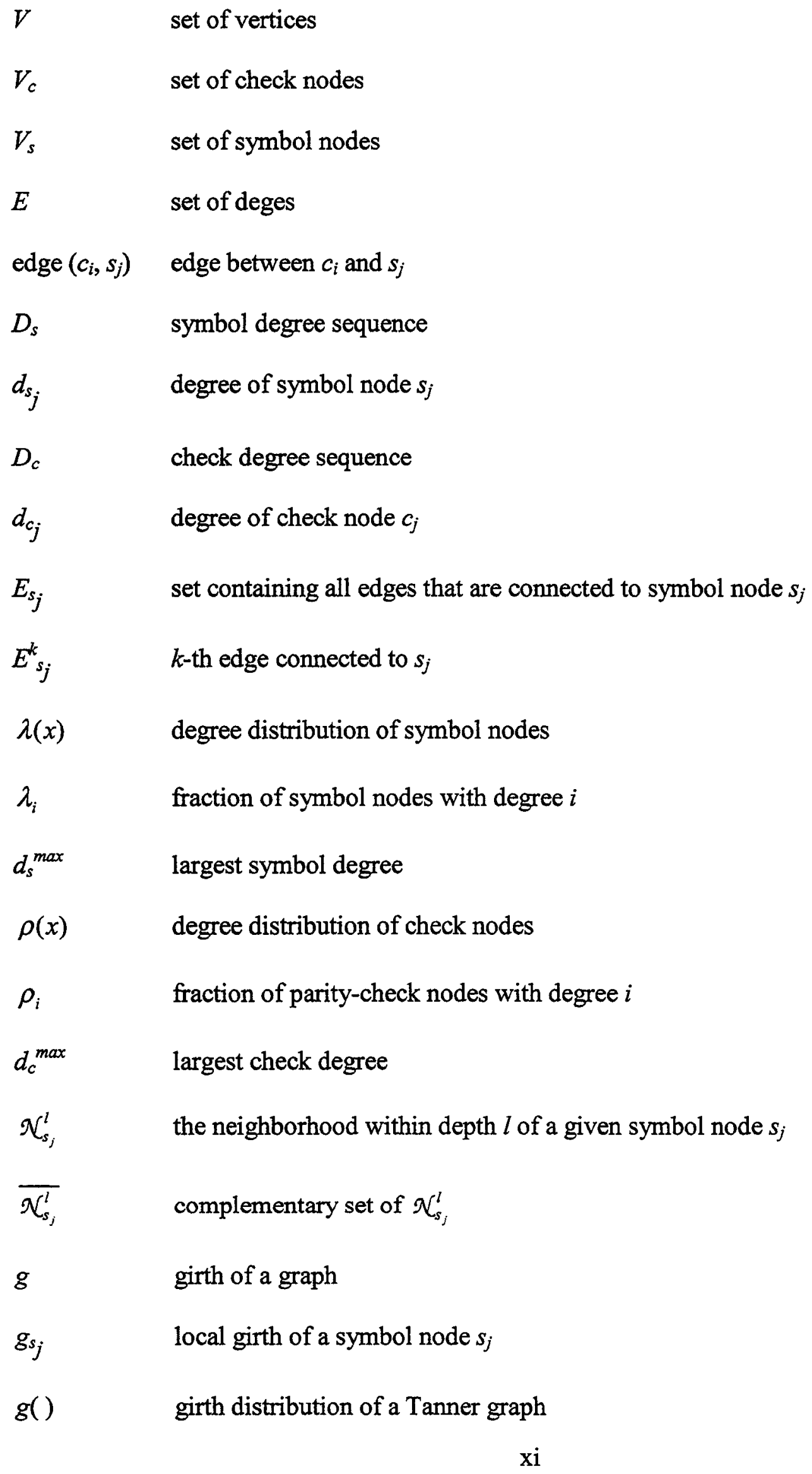




$\begin{array}{ll}V_{s}^{p} & \text { redundant subset of symbol nodes } \\ V_{s}^{d} & \text { information subset of symbol nodes } \\ p_{C}\left(c_{i}\right) & \text { probability mass function of sending codewords } \\ f_{R}(r) & \text { probability density function of receiving } r \\ p_{C}(c \mid r) & \text { conditional probability of transmitted signal } c, \text { knowing received signal } r \\ f_{R}(r \mid c) & \text { conditional probability density function of receiving signal } r, \text { knowing } \\ \text { transmitted signal } c \\ N_{0} & \text { one sided noise spectral density } \\ \sigma & \text { noise variance } \\ E_{b} & \text { average energy per information bit } \\ m_{s \rightarrow c} & \text { output message of a symbol node } s \text { passed to check node } c \\ m_{c \rightarrow s} & \text { output message of a check node } c \text { passed to symbol node } s \\ N(c) \backslash s & \text { set of all neighboring symbol nodes of } c \text { other than } s \\ N(s) \backslash c & \text { set of all neighboring check nodes of } s \text { other than } c \\ P(E) & \text { probability that the receiver accepts a packet containing errors } \\ P_{e} & \text { probability of undetected error } \\ P_{r} & \text { probability of detected error } \\ N_{i} & \text { throughput } \\ p_{i} & \text { total number of bits transmitted after the } i \text { th transmission } \\ & \end{array}$

$N_{a v} \quad$ average total number of bits that must be transmitted in order for the information frame to be accepted by the receiver 
$F_{i} \quad$ the frame error rate of the code used in the $i$ th transmission

$H_{\text {ext }} \quad$ extended parity-check matrix

$h_{\text {ext }} \quad$ extension matrix

$\mathrm{C}^{*} \quad$ capacity of a binary-input, AWGN channel 


\section{Motivation and Outline of Thesis}

The theory of error correcting codes started as a subject in Electrical Engineering with the pioneering work of Claude Shannon in 1948, on achieving reliable communication over a noisy transmission channel. Shannon's central theme was that with a sufficiently sophisticated channel encoder and decoder, we can achieve reliable communication over the channel at a rate up to the channel capacity. Since then, the problem of reliable communication in the presence of noise has changed to finding good codes and designing efficient decoding methods that work very close to channel capacity. The development of turbo codes and Low-Density Parity-Check (LDPC) codes in recent years has opened a brand new way of constructing capacity approaching codes that can be decoded with feasible complexity.

On the other hand, in the last few years, the demand for efficient and reliable digital transmission has tremendously increased. A powerful and flexible error control scheme is required for high speed data services in wireless communications and data networks to meet different code rate and quality of service requirements of these applications. In order to provide such levels of flexibility and reliability, automatic repeat request (ARQ) schemes can be combined with FEC channel coding (Hybrid-ARQ). In brief, FEC coding takes care of the most frequent error patterns caused by the noise on the channel, while retransmission takes care of burst errors occurred on the channel. In particular, type-II hybrid-ARQ protocols based on rate-compatible (RC) codes both inherit a flexible structure and have the potential of providing near capacity throughput performance with employing a family of powerful RC codes. For example, the Type-II hybrid-ARQ protocols based on rate-compatible punctured turbo (RCPT) codes have demonstrated near capacity 
performance, even at short block lengths. Codes with short block lengths are desirable for practical systems where we want to limit the encoding and decoding complexity and delay. However, the high decoding complexity of turbo codes results in high costs for commercial systems. LDPC codes are the other type of capacity approaching codes which are believed to be serious competitors for turbo codes with less decoding complexity.

These facts have motivated us to investigate the performance of Type-II hybrid-ARQ protocols based on RC-LDPC codes at short block lengths and the results of this research are presented in the following 4 chapters: In chapter 1, after briefly explaining block codes, we introduce LDPC codes. Different methods of constructing LDPC codes together with their encoding and decoding are explained in chapter 2. In particular, we describe the Progressive-Edge Growth (PEG) construction which has been recently shown to be capable of constructing very good irregular LDPC codes at short block lengths with a linear-time encoding complexity. In chapter 4 , we first explain the analysis and equations that govern the type-II hybrid ARQ protocols based on RC codes and then describe the type-II hybrid ARQ protocol based on RCPT codes and regular LDPC codes. After that, we will thoroughly describe the construction method of a family of irregular RCLDPC codes by a combination of puncturing and extending techniques. We introduce a suitable structure for extended parity-check matrices which provides a good connection between the old and new parts of each extended matrix. Finally, we compare the throughput of our system with the throughput of similar systems based on turbo codes and regular LDPC codes. 


\section{Chapter 1}

\section{Low-Density Parity-Check}

(LDPC) Codes 
Error control codes increase the noise immunity of transmitted information by inserting controlled redundancy into the information stream, which allows the receiver to detect and possibly correct the errors. In this chapter, we first explain block codes and then the most easily implemented and therefore the most widely used block codes, linear block codes, are described. Finally, we conclude the chapter by introducing low-density paritycheck (LDPC) codes.

\section{1-1 Block Codes}

Let $A=\left\{a_{1}, a_{2}, \ldots, a_{q}\right\}$ be a finite set, called a code alphabet, and let $A^{n}$ be the set of all strings of length $n$ over $A$. Any non-empty subset $C$ of $A^{n}$ is called a q-ary block code and each string in $C$ is called a codeword. If $C \subset A$ contains $M$ codewords, then it is customary to say that $C$ is an $(n, M)$-code. The rate of a $q$-ary $(n, M)$-code is

$$
R=\frac{\log _{q} M}{n}
$$

Hereafter, we assume that the output of the information source is a sequence of binary digits 0 and 1 . So we can take $A=\{0,1\}$ and the code is binary. For binary block codes, in encoder, the binary information sequence is segmented into message blocks of length $k$ and then according to a one-by-one correspondence, each of message blocks is transformed to a codeword.

A block code of length $n$ and $2^{k}$ codewords is called a linear $(n, k)$ code if its $2^{k}$ codewords form a $k$-dimensional subspace of the vector space of all $n$-tuples over the Galois field $G F(2)$. 
An $(n, k)$ linear block code can be generated by a set of $k$ linearly independent binary $n$-tuples $g_{0}, g_{1}, \ldots, g_{k-1}$. The codewords are obtained by linearly combining these $n$ tuples. Consequently, the codeword for a message $\boldsymbol{m}=\left(m_{0}, m_{1}, \ldots, m_{k-1}\right)$ can be represented as

$$
\boldsymbol{c}=m_{0} \boldsymbol{g}_{0}+m_{1} \boldsymbol{g}_{1}+\ldots+m_{k-1} \boldsymbol{g}_{k-1}
$$

The vectors $\boldsymbol{g}_{0}, \boldsymbol{g}_{1}, \ldots, \boldsymbol{g}_{k-1}$ can be arranged as rows of a $k \times n$ matrix as follows

$$
\boldsymbol{G}=\left(\begin{array}{c}
\boldsymbol{g}_{0} \\
\boldsymbol{g}_{1} \\
\vdots \\
\boldsymbol{g}_{k-1}
\end{array}\right)=\left(\begin{array}{cccc}
g_{0,0} & g_{0,1} & \cdots & g_{0, n-1} \\
g_{1,0} & g_{1,1} & \cdots & g_{1, n-1} \\
\vdots & \vdots & \ddots & \vdots \\
g_{k-1,0} & g_{k-1,1} & \cdots & g_{k-1, n-1}
\end{array}\right)
$$

The matrix $G$ is called the generator matrix of the code. The corresponding codeword $c$ of the message $m$ can therefore be written as

$$
c=m G
$$

Note that any $k$ linearly independent codewords can form a generator matrix and thus the generator matrix of a linear block code is not unique.

\section{Example 1.1}

The set of 4 linearly independent 7-tuples

$g_{0}=1101000, g_{1}=0110100, g_{2}=1110010$, and $g_{3}=1010001$, generates a $(7,4)$ linear block code with the following generator matrix

$$
G=\left(\begin{array}{lllllll}
1 & 1 & 0 & 1 & 0 & 0 & 0 \\
0 & 1 & 1 & 0 & 1 & 0 & 0 \\
1 & 1 & 1 & 0 & 0 & 1 & 0 \\
1 & 0 & 1 & 0 & 0 & 0 & 1
\end{array}\right) .
$$




\section{1-2 Systematic Linear Block Codes}

Consider a linear block code with generator matrix $G$. Using Gaussian elimination and column ordering, it is always possible to obtain a generator matrix with the following form

$$
G_{k \times n}=\left(P_{k \times(n-k)} \vdots I_{k}\right)
$$

where $I_{k}$ is the $k \times k$ identity matrix and $P$ is a $k \times(n-k)$ binary matrix of the form

$$
P=\left(\begin{array}{cccc}
p_{0,0} & p_{0,1} & \cdots & p_{0, n-k-1} \\
p_{1,0} & p_{1,1} & \cdots & p_{1, n-k-1} \\
\vdots & \vdots & \ddots & \vdots \\
p_{k-1,0} & p_{k-1,1} & \cdots & p_{k-1, n-k-1}
\end{array}\right)
$$

Clearly, when a data block is encoded using a systematic generator matrix, it is embedded without any modification in the last $k$ coordinates of the resulting codeword:

$$
\begin{aligned}
c & =\boldsymbol{m} G \\
& =\left(m_{0} m_{1} \cdots m_{k-1}\right)(P \vdots I) \\
& =\left(c_{0} c_{1} \cdots c_{n-k-1} \vdots m_{0} m_{1} \cdots m_{k-1}\right) .
\end{aligned}
$$

Also, after the decoding, the $k$ last bits of the estimated codeword are passed along to the data sink. Notice that the generator matrix given in Example $\mathbf{1 . 1}$ is in systematic form.

\section{1-3 Dual Code and Parity-Check Matrix}

Since a $q$-ary code $C$ of length $n$ is a subspace embedded within the vector space of all $n$-tuples over $G F(q)$, we can meaningfully talk about the dual space of $C$. The dual space of a linear block code $C$ is called the dual code of $C$ and is denoted by $C^{\perp}$. Clearly, $C^{\perp}$ is a vector space of dimension $(n-k)$. Let $\left\{\boldsymbol{h}_{0}, \boldsymbol{h}_{1}, \ldots, \boldsymbol{h}_{n-k-1}\right\}$ be a basis for $C^{\perp}$, meaning that every codeword of $C^{\perp}$ can be written as a linear combination of $\left\{\boldsymbol{h}_{0}, \boldsymbol{h}_{1}, \ldots, \boldsymbol{h}_{n-k-1}\right\}$. 
Now we can consider an $(n-k) \times n$ matrix $H$ with $(n-k)$ linearly independent rows, called parity check matrix

$$
H=\left(\begin{array}{c}
\boldsymbol{h}_{0} \\
\boldsymbol{h}_{1} \\
\vdots \\
\boldsymbol{h}_{n-k-1}
\end{array}\right)=\left(\begin{array}{cccc}
h_{0,0} & h_{0,1} & \cdots & h_{0, n-1} \\
h_{1,0} & h_{1,1} & \cdots & h_{1, n-1} \\
\vdots & \vdots & \ddots & \vdots \\
h_{n-k-1,0} & h_{n-k-1,1} & \cdots & h_{n-k-1, n-1}
\end{array}\right)
$$

with the property that any vector in row space of $G$ is orthogonal to any row of $H$ and any vector that is orthogonal to all the rows of $H$ is in row space of $G$. Thus, for any codeword $c$ we can write

$$
c H^{\mathrm{T}}=\mathbf{0} .
$$

One can easily see that if the generator matrix of a code is in the systematic form of (1.5), the parity-check matrix may take the following form

$$
H_{(n-k) \times n}=\left(I_{n-k} \vdots P_{(n-k) \times k}^{\mathrm{T}}\right) \text {, }
$$

where $P^{\mathrm{T}}$ denotes the transpose of the matrix $P$.

\section{Example 1.2}

Here is the parity-check matrix of the code given in Example 1.1

$$
H=\left(\begin{array}{lllllll}
1 & 0 & 0 & 1 & 0 & 1 & 1 \\
0 & 1 & 0 & 1 & 1 & 1 & 0 \\
0 & 0 & 1 & 0 & 1 & 1 & 1
\end{array}\right)
$$

It can easily be verified that $G H^{\mathrm{T}}=0$, which implies that the row spaces of generator and parity-check matrices are orthogonal. 


\section{1-4 Minimum Distance of a Block Code}

Let us consider an $(n, k)$ linear block code. The number of nonzero elements of a codeword $c$ is called the Hamming weight of that codeword. The Hamming distance, $d\left(c_{1}\right.$, $c_{2}$ ), between two codewords $c_{1}$ and $c_{2}$ is defined as the number of locations in which the two codewords differ. The minimum distance of a code, $d_{\min }$, is defined as the smallest Hamming distance between two different codewords in the code.

One can see that the Hamming distance between two codewords is identical to the weight of their modulo-2 sum. The linearity property of block codes requires that the modulo-2 sum of any two codewords is another codeword. This implies that the minimum distance of a linear block code is the smallest Hamming weight of the nonzero codewords in the code. The importance of the minimum distance parameter is in the fact that it determines error detection and error correction capabilities of a code: a code $C$ with minimum distance $d_{\min }$ is $\left(d_{\min }-1\right)$-error-detecting and $\left\lfloor\left(d_{\min }-1\right) / 2\right\rfloor$-errorcorrecting. For example, a code with $d_{\min }=5$ can detect all error patterns of Hamming weights up to 4. Meanwhile, such a code can correct all error patterns of Hamming weights not exceeding 2 .

\section{1-5 Low-Density Parity-Check (LDPC) Codes}

An LDPC code [1] is a linear block code and therefore can be fully described by its parity-check matrix. Parity-check matrices of LDPC codes are sparse; that is, they consist mainly of 0 's and a small number of 1's. In regular LDPC codes, column and row weights of the parity-check matrices are fixed while in irregular LDPC codes, the weights are chosen according to some distribution. In particular, a $\left(d_{s}, d_{c}\right)$-regular LDPC 
code $C$ of length $n$, has a parity-check matrix $H$ with $n$ columns of Hamming weight $d_{s}$ and $m:=\frac{n d_{s}}{d_{c}}$ rows of Hamming weight $d_{c}$.

LDPC codes, as any other linear block code, can be represented by Tanner graphs [2]. A Tanner graph is a bipartite graph in which one set of nodes, the symbol nodes (variable nodes), corresponds to the code symbols and the other set of nodes, the check nodes, corresponds to the set of parity-check constraints which define the code. An edge connects a symbol node $s_{j}$ and a check node $c_{i}$ if and only if $s_{j}$ appears in the parity-check equation corresponding to $c_{i}$, or in other words, if and only if a ' 1 ' is located at position $(i, j)$ of $H$. For a $\left(d_{s}, d_{c}\right)$-regular LDPC code of length $n$, the Tanner graph has $n d_{s}=m d_{c}$ edges, $d_{s}$ edges incident to each symbol node and $d_{c}$ edges incident to each check node.

As an example, consider the following parity-check matrix which defines a $(3,5)$ regular LDPC code of length 10.

$$
H=\left(\begin{array}{llllllllll}
1 & 1 & 0 & 1 & 0 & 1 & 0 & 0 & 1 & 0 \\
0 & 1 & 1 & 0 & 1 & 0 & 1 & 1 & 0 & 0 \\
1 & 0 & 0 & 0 & 1 & 1 & 0 & 0 & 1 & 1 \\
0 & 1 & 1 & 1 & 0 & 1 & 1 & 0 & 0 & 0 \\
1 & 0 & 1 & 0 & 1 & 0 & 0 & 1 & 0 & 1 \\
0 & 0 & 0 & 1 & 0 & 0 & 1 & 1 & 1 & 1
\end{array}\right) .
$$

The Tanner graph corresponding to the above parity-check matrix is shown in Fig. 1.1. Note that each symbol node corresponds to one column of $H$ and each check node represents one row of $H$. In this example, every symbol node has degree 3 and every check node has degree 5 . 


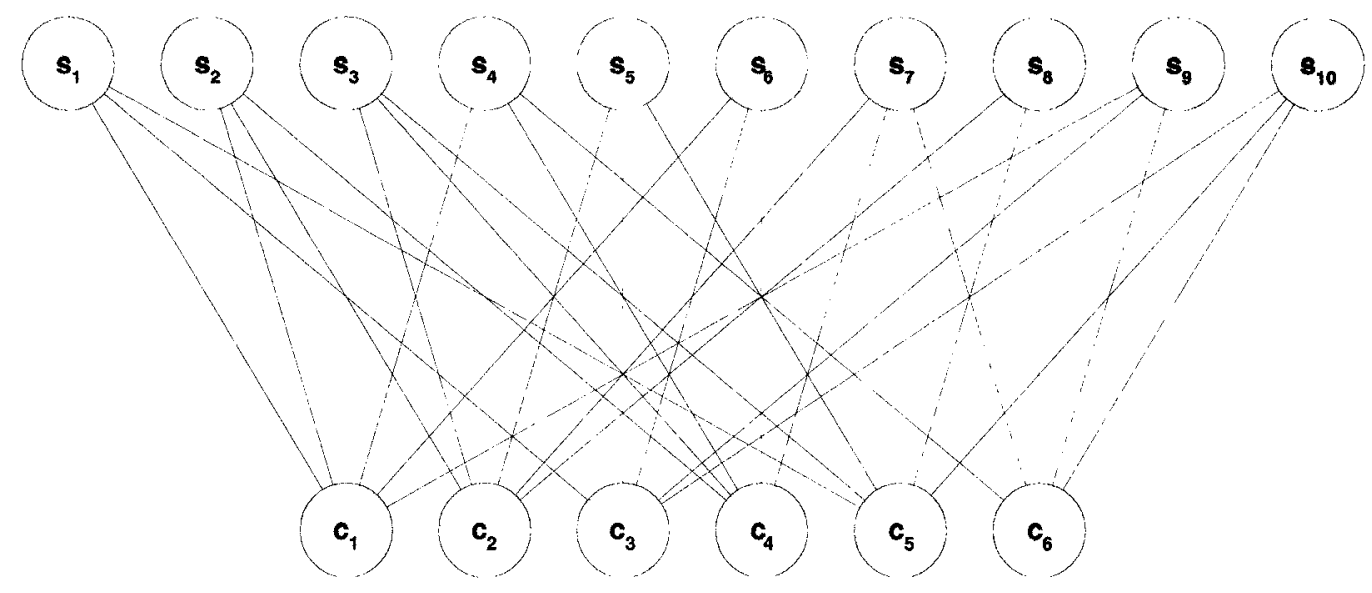

Fig. 1.1. Tanner graph of the $(3,5)$-regular LDPC code

In Chapter II, we will see that iterative decoding algorithms for LDPC codes can naturally be described using the Tanner graph representation of these codes.

\section{1-6 Comparison of LDPC Codes and Turbo-Codes}

LDPC codes were introduced by Gallager in his thesis in 1960 [1]. They were soon forgotten however, due to limited computational resources available at the sixties, which made the corresponding iterative decoding algorithms look impractical. Recently, following the successful introduction of parallel concatenated convolutional codes known as turbo-codes [3], interest in LDPC codes has been rekindled, as researchers have realized the phenomenal strength of iterative coding schemes and the attractive performance/complexity tradeoff that they offer. The past few years in particular, have witnessed a great amount of research devoted to these schemes [4]-[7]. It is now well known that both classes of turbo-codes and LDPC codes exhibit a performance extremely close to the best possible as determined by Shannon capacity formula. 
LDPC codes have some important advantages over turbo-codes. First, iterativedecoding algorithms for LDPC codes, including belief-propagation $(B P)$ and min-sum, are fully parallelizable and thus may potentially be realized at significantly greater speeds compared to turbo-codes. Second, low complexity, yet high performance decoders, other than BP, can be designed for these codes [8]. Third, convergence of LDPC decoder to a correct codeword is usually a detectable event. One drawback of LDPC codes is that for long block lengths, the encoding complexity is generally high.

Anyhow, in the coding community, it is now widely accepted that in many applications, LDPC codes are serious competitors for turbo-codes and more research is underway to analyze, design, and efficiently implement LDPC encoders and decoders. 


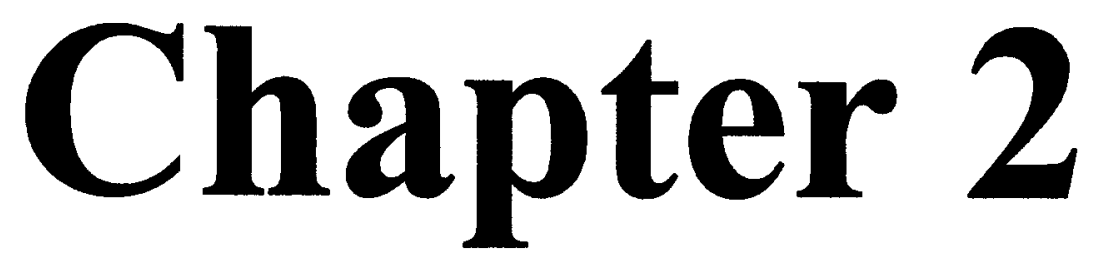

\section{Constructing, Encoding,}

and Decoding of LDPC

Codes 
In transmission systems we require some codes which allow the transmission of information close to capacity at low probability of error and which are efficiently encodable and decodable. It has been shown that LDPC codes can achieve performances very close to theoretical limits with modest decoding complexity. As such, encoding and decoding of LDPC codes have been the subject of a vast research in recent years. In this chapter we first describe different methods of constructing LDPC codes and the encoding complexity of LDPC codes. Then we explain iterative decoding algorithms as efficient algorithms for decoding LDPC codes. Finally, the performance of LDPC codes constructed with different constructions will be compared with each other.

\section{2-1- Constructing and Encoding of LDPC Codes}

In this section we first introduce some necessary definitions and notations on the graph. In this part we largely follow the notations and definitions of [16]. Then different ways of constructing LDPC codes are explained.

\section{2-1-1 Some Definitions and Notations}

The parity-check matrices of LDPC codes are sparse matrices constructed subject to constraints on the weights of rows and each columns. A bipartite Tanner graph can be used to uniquely define the parity-check matrix. Formally, A Tanner graph is defined as $G=(V, E)$, Where $V=V_{c} \cup V_{s}$ is the set of vertices with $V_{c}=\left\{c_{0}, c_{1}, \ldots, c_{m-1}\right\}$ being the set of check nodes and $V_{s}=\left\{s_{0}, s_{1}, \ldots, s_{n-1}\right\}$ the set of symbol nodes. Set $E$ is the set of edges in $V_{c \times} V_{s}$, with edge $\left(c_{i-1}, s_{j-1}\right) \in E$ if and only if $h_{i, j} \neq 0,1 \leq i \leq m, 1 \leq j \leq n$, where $h_{i, j}$ is the element in the $i$ th row and $j$ th column of $\mathrm{H}$. In a $\left(d_{s}, d_{c}\right)$-regular Tanner graph every symbol node is connected to $d_{s}$ check nodes and every check node is connected to $d_{c}$ 
symbol nodes. Suppose that $D_{s}=\left(d_{s_{0}}, d_{s_{1}}, \ldots, d_{s n-1}\right)$ is the symbol degree sequence in which $d_{s_{j}}$ is the degree of symbol node $s_{j}, 0 \leq j \leq n-1$, in non-decreasing order, i.e., $d_{s_{0}} \leq$ $d_{s_{1}} \leq \ldots \leq d_{s n-1}$, and $D_{c}=\left(d_{c_{0}}, d_{c_{1}}, \ldots, d_{c m-1}\right)$ is the check degree sequence in which $d_{c_{j}}$ is the degree of parity-check node $c_{j}, 0 \leq j \leq m-1$, and $d_{c_{0}} \leq d_{c_{1}} \leq \ldots \leq d_{c m-1}$. Also suppose that the set of edges, $E$, is partitioned in terms of $V_{s}$ as $E=E_{s_{0}} \cup E_{s_{1}} \cup \ldots \cup E_{s n-1}$, in which $E_{s_{j}}, 0 \leq j \leq n-1$, contains all edges that are connected to symbol node $s_{j}$. Further, denote the $k$-th edge connected to $s_{j}$ by $E_{s_{j}}^{k}, 0 \leq k \leq d_{s_{j}}-1$. Fig. 2.1 shows an example of an irregular Tanner graph with $D_{s}=\{2,2,2,2,3,3,3,4\}$ and $D_{c}=\{4,5,6,6\}$.

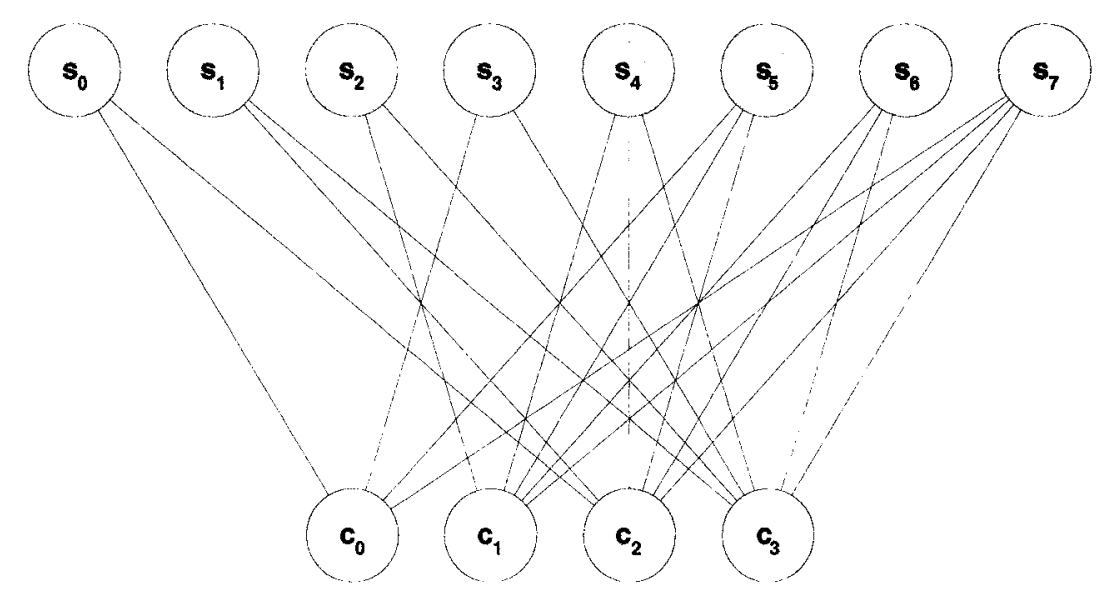

Fig. 2.1. An irregular Tanner graph with $D_{s}=\{2,2,2,2,3,3,3,4\}$ and $D_{c}=\{4,4,5,6\}$

In general, an ensemble of Tanner graphs, and correspondingly an ensemble of LDPC codes, is determined by its degree distribution pairs; the degree distribution of symbol nodes is defined as $\lambda(x)=\sum_{i \geq 1}^{d_{s}^{m i x}} \lambda_{i} x^{i}$, where $\lambda_{i}$ is the fraction of symbol nodes with degree $i, d_{s}^{\max }$ is the largest entry in $D_{s}=\left(d_{s_{0}}, d_{s_{1}}, \ldots, d_{s n-1}\right)$ and $\sum_{i \geq 1}^{d_{i m}^{\max }} \lambda_{i}=1$. Similarly the degree 
distribution of parity-check nodes is defined as $\rho(x)=\sum_{i \geq 2}^{d_{c}^{\max }} \rho_{i} x^{i}$, where $\rho_{i}$ is the fraction of parity-check nodes with degree $i, d_{c}^{\max }$ is the largest entry in $D_{c}=\left(d_{c_{0}}, d_{c_{1}}, \ldots, d_{c m-1}\right)$, and $\sum_{i \geq 2}^{d_{c}^{\max }} \rho_{i}=1$

A path between two nodes $u$ and $v$ of a graph is defined as a sequence of distinct nodes which begins with $u$, ends in $v$, and there is an edge between any two consecutive nodes of the sequence. A cycle is defined as a closed path which begins from a node $u$ and ends with itself. When there is a path between two nodes $u$ and $v$ of a graph, then we say $u$ can be reached by $v$ and vice versa. In a Tanner graph, the neighborhood within depth $l$ of a given symbol node $s_{j}, \mathcal{N}_{s_{j}}^{l}$, is defined as the set of all check nodes that can be reached by traversing at most $2 l+1$ edges, on a tree spreading from symbol node $s_{j}$. The complementary set of $\mathcal{N}_{s_{j}}^{l}, \quad \overline{\mathcal{K}_{s_{j}}^{l}}, \quad$ is defined as $\quad V_{c} \backslash \mathcal{N}_{s_{j}}^{l}$, i.e. $\mathcal{N}_{s_{j}}^{l} \cup \overline{\mathfrak{N}_{s_{j}}^{l}}=V_{c}$ and $\mathcal{N}_{s_{j}}^{l} \cap \overline{\mathcal{N}_{s_{j}}^{l}}=\phi$. Similarly the neighborhood within depth $l$ of a given check node $c_{i}, \mathcal{N}_{c_{l}}^{l}$, is defined as the set of all parity-check nodes that can be reached by traversing at most $2 l$ edges, on a tree spreading from $c_{i}$. Figure 2.2 and Figure 2.3 show the tree spreading from a symbol node $s_{j}$ and check node $c_{i}$, respectively.

The girth of a graph is defined as the number of edges of the shortest cycle in the graph and is denoted by. $g$. On the Tanner graph, the local girth of each symbol node $s_{j}$, $g_{s_{j}}$, is defined as the length of the shortest cycle passing through that symbol node. Obviously, for a Tanner graph we have $g=\min _{j}\left\{g_{s_{j}}\right\}$. The girth distribution of a Tanner 
graph is defined as the function $g(l), l=4,6,8, \ldots, l_{\max }$, which gives the fraction of the symbol nodes with girth $l$, where $l_{\max }$ is the maximum girth in the graph [9].

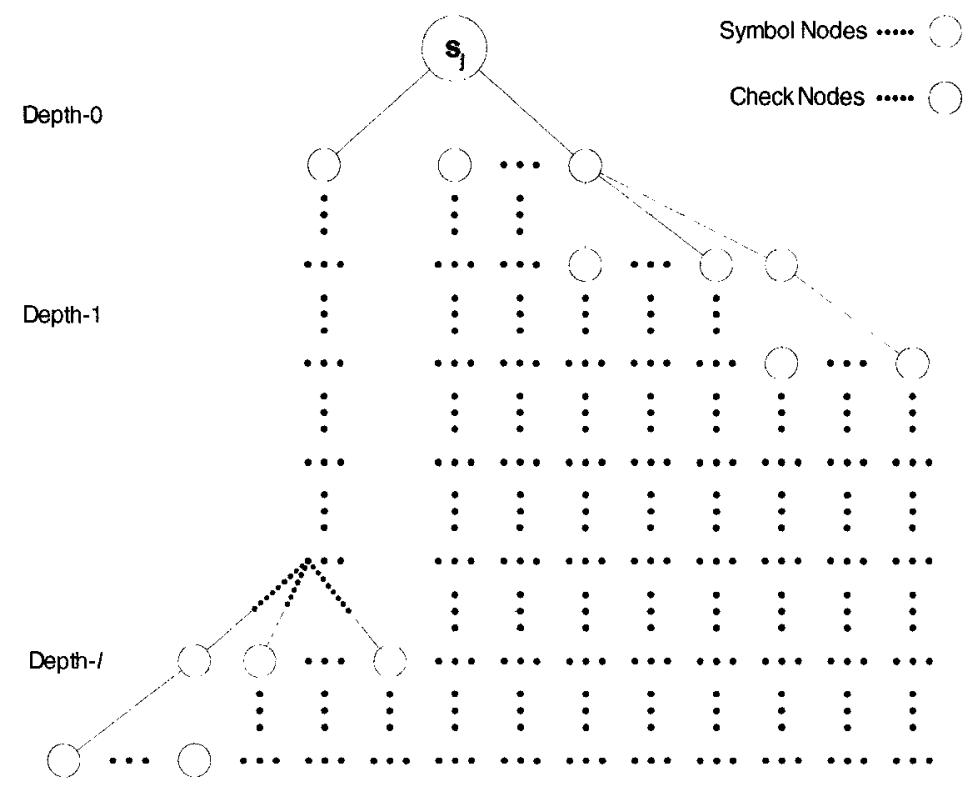

Fig. 2.2. The tree spreading from a symbol node $\mathbf{s}_{\mathbf{j}}$

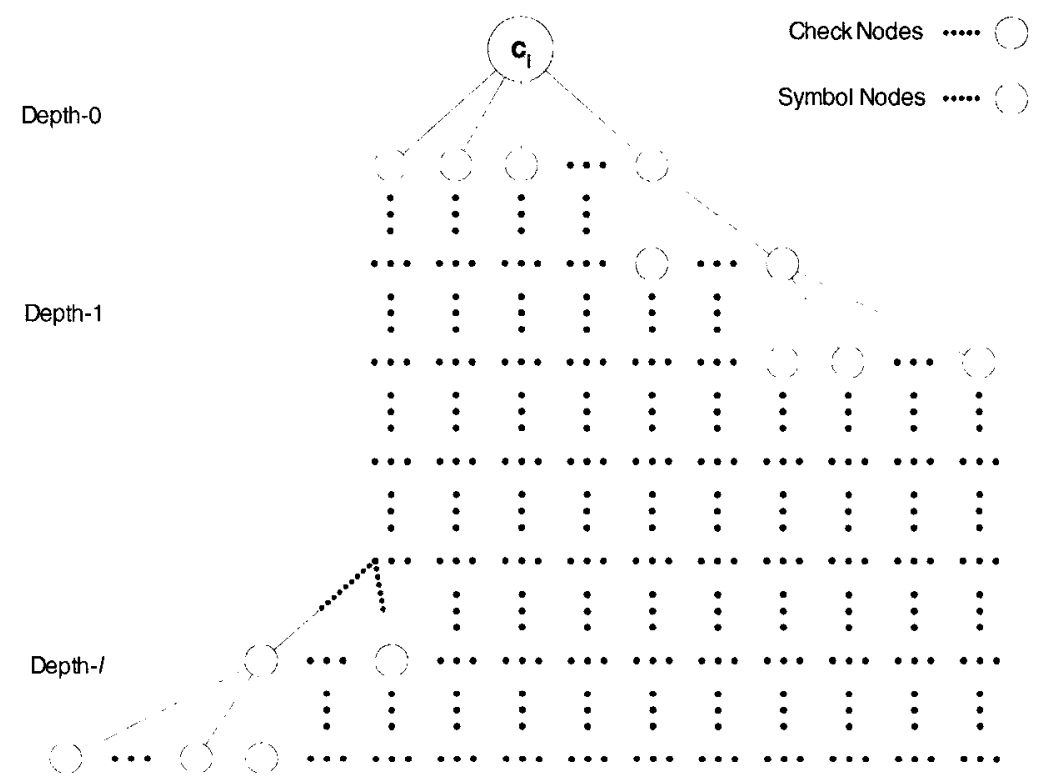

Fig. 2.3. The tree spreading from a check node $\mathbf{c}_{\mathbf{i}}$ 


\section{2-1-2- Different Constructions of LDPC Codes}

There are different methods which can be used for constructing LDPC codes. These construction methods can be divided into two main categories: algebraic methods and random methods. The algebraic category includes systematic methods such as finite geometry based constructions [10] and group-theoretic based constructions [11], [12]. For example, the finite geometry based constructions allow us to construct LDPC codes, with relatively good minimum distances, based on the lines and points of a finite geometry. However, applications of algebraic constructed codes are usually limited to specified code rates and block sizes, which is not the case for random construction methods. In this subsection we focus on random constructions; we first explain two well-known random constructions and then describe a new general method which can be used to generate LDPC codes with very good performances at short block lengths. The version of algorithm that we describe here is completely deterministic. However, as it will be described, the method also has a random version which is slightly different from deterministic version.

In fact, the determination of an LDPC code has two steps: first a profile is selected that describes the desired number of columns of each weight and rows of each weight in the parity-check matrix. As it was explained, each nonzero entry in the parity-check matrix corresponds to an edge connecting a symbol node to a check node of the Tanner graph. Thus, the profile specifies the degrees of vertices in the Tanner graph.

Second a construction method is chosen that determines an algorithm for putting edges between the vertices of Tanner graph in a way that satisfies the constraints. In this section we describe three different construction methods for a fixed profile. 
Permutation construction- Regular parity-check matrices can be built by superposing random permutation matrices [1]. In fact, this method can be used to easily obtain an ensemble of regular LDPC codes. Consider the special case of $(3,4)$-regular LDPC code with $n=20$ and $m=15$ as shown in Fig. 2.4.

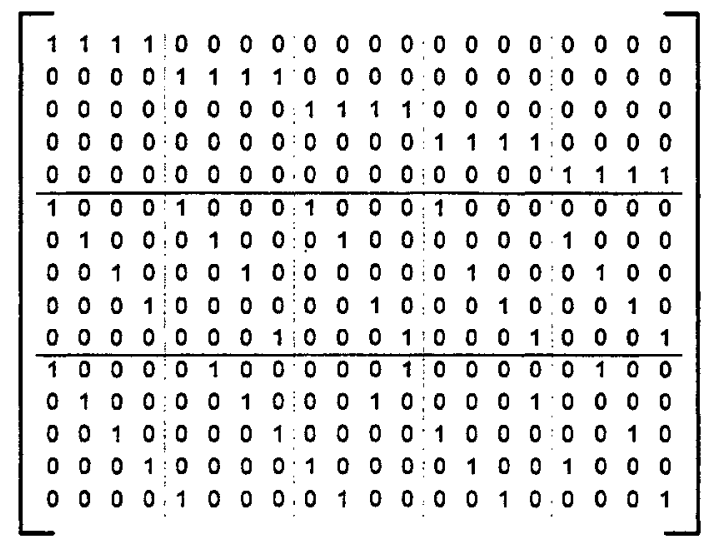

Fig. 2.4. Parity-check matrix of a (3, 4)-regular LDPC code with $n=20$ and $m=15$

The matrix is divided into $d_{s}$ sub-matrices, each containing a single 1 in each column. The first of these sub-matrices contains all its 1 's in descending order; that is, the $i$ th row contains 1 's in columns $(i-1) d_{c}+1$ to $i d_{c}$. The other sub-matrices are merely column permutation of the first. The ensemble of $(3,4)$-regular LDPC codes is obtained by random permutations of the bottom 2 sub-matrices of a matrix such as the one in Fig. 2.4.

Poisson Construction- In this method we put the edges of Tanner graph completely at random, subject to the profile constraints. Obviously, we can not place two edges between one pair of vertices, since this would correspond to a double entry in the paritycheck matrix [13]. One way for implementation of a Poisson construction is to make a list of all columns, containing each column with multiplicity equal to its degree and a similar 
list of all rows, containing each row with a multiplicity equal to its degree and then use a random permutation to map one list to the other, while taking care of duplicate entries.

Progressive Edge-Growth (PEG) Construction [14]-[16]- It is known that the BP algorithm provides the best performance, in terms of minimizing bit error rate (BER), for cycle-free Tanner graphs. As such, it is natural to try to lessen the influence of the cycles on the iterative decoding process as much as possible. One way for achieving this goal is to construct LDPC codes that have the largest possible girth distribution, or in other words, to have more symbol nodes with larger girths [9]. In both of the Permutation method and Poisson method, the Tanner graph is randomly constructed, usually by avoiding cycles of length 4 . However there is no guarantee that any given random graph defines a good code having a suitable girth distribution that facilitates iterative decoding. An efficient method for constructing Tanner graphs with a large average girth, in a greedy sense, is PEG construction, in which the edges are progressively established between symbol and check nodes of the Tanner graph in a edge-by-edge manner [14]-[16].

In fact, constructing a Tanner graph with the largest possible girth is a rather difficult combinatorial problem, and thus we have to look for sub-optimal algorithms that are feasible in practice and construct a Tanner graph with a relatively large girth. The PEG algorithm is one such algorithm that maximizes the local girth of a symbol node whenever a new edge is added to this symbol node.

Suppose that the edges of the first $j$ symbol nodes of a Tanner graph, $E_{s_{0}} \cup E_{s_{1}} \cup \ldots \cup$ $E_{s_{j-1}}$, have been established and let $g^{t}=\min \left\{g_{s_{0}}, g_{s_{1}}, \ldots, g_{s_{j-1}}\right\}$. In order to construct a graph having a large girth, the edge set $E_{s_{j}}$ of symbol node $s_{j}$ have to be chosen such that adding these new edges to the current graph setting maintains the local girth of $s_{j}$ at its 
maximum possible value. For this purpose, the edges of $E_{s_{j}}$ should be optimized in terms of maximizing the local girth $g_{s j}$, because if adding $E_{s_{j}}$ to the current graph generates a cycle shorter than $g^{t}$, this new short cycle must pass through symbol node $s_{j}$.

To find the optimum set $E_{s_{j}}$, corresponding to the symbol node $s_{j},\left(\begin{array}{c}m \\ d_{s_{j}}\end{array}\right)$ different combinations of check nodes need to be checked, where $\left(\begin{array}{c}m \\ d_{s_{j}}\end{array}\right)$ is a binomial coefficient, which makes it impossible even for moderate values of $m$. The PEG algorithm is a best effort algorithm in which $d_{s_{j}}$ edges of $E_{s_{j}}$ are added to the current graph on a edge-byedge basis such that whenever a new edge is being added to $s_{j}$, the length of the shortest cycle passing through symbol node $s_{j}$ is maximized. To do this, each time a new edge of $s_{j}$ is being determined, the tree originating from symbol node $s_{j}$ up to depth $l$ is spread such that $\mathcal{N}_{s_{j}}^{l} \neq \varnothing$ but $\mathcal{N}_{s_{j}}^{l+1}=\varnothing$, or the cardinality of $\mathcal{N}_{s_{j}}^{l}$ stops increasing but is smaller than $m$. Then an edge is placed between $s_{j}$ and a check node $c_{i}$ selected from the set $\overline{\mathcal{N}_{s_{j}}^{l}}$, where $c_{i}$ has the lowest check node degree under the current graph settings $E_{s_{0}} \cup E_{s_{1}} \cup \ldots$ $u E_{s-1}$. This special selection of $c_{i}$ is made in accordance with the evidence that a concentrated check-degree sequence is optimum in some weak sense [17], [18]. Also when there are multiple check nodes in $\overline{\mathfrak{K}_{s_{j}}^{l}}$ which have the smallest check degree under the current graph settings, we select the one with the smallest subscript; this approach makes the algorithm completely deterministic. However, in [16] this selection is made by randomly choosing one of the check nodes which has the smallest degree. In the following we pre- 
sent the algorithm by a flowchart, in which the edge between a check node $c_{i}$ and a symbol node $s_{j}$ is represented by "edge $\left(c_{i}, s_{j}\right)$ " [16]:

\section{Deterministic PEG Algorithm:}

For $j=0$ to $\mathrm{n}-1 \mathrm{do}$

begin

for $k=0$ to $d_{s_{j}}-1$ do

begin

if $k=0$

$E_{s_{j}}^{k} \leftarrow \operatorname{edge}\left(c_{i}, s_{j}\right)$, where $E_{s_{j}}^{0}$ is the first edge incident to $s_{j}$ and $c_{i}$ is the check node which has smallest check degree under the current graph setting $E_{s_{0}} \cup E_{s_{1}}$ $\cup \ldots \cup E_{s_{j-1}}$. If they are multiple choices for $c_{i}$, we select the one with smallest subscript.

else

Expanding a tree from symbol node $s_{j}$ up to depth $l$ under the current graph setting such that $\overline{\mathcal{K}_{s_{j}}^{l}} \neq \varnothing$ but $\overline{\mathcal{K}_{s_{j}}^{l+1}}=\varnothing$, or the cardinality of $\mathcal{N}_{s_{j}}^{l}$ stops increasing but is less than $m$, then $E_{s_{j}}^{k} \leftarrow \operatorname{edge}\left(c_{i}, s_{j}\right)$, where $E_{s_{j}}^{k}$ is the $k$-th edge incident to $s_{j}$ and $c_{i}$ is the check node picked from the set $\overline{\mathcal{K}_{s_{j}}^{l}}$ which has smallest check degree. If they are multiple choices for $c_{i}$, we select the one with smallest subscript.

end

end 


\section{2-1-3- Encoding of LDPC codes}

Unless special measures are taken during construction of the parity-check matrix of LDPC codes, for the purpose of encoding it is necessary to transfer $H$ into $H_{s y s}$, the equivalent systematic form of $H$. This can be accomplished by Gaussian elimination. In order to store $G_{s y s}$ corresponding to $H_{s y s}$ in the encoder a considerable amount of memory is required because $H_{s y s}$ is not sparse even though $H$ is usually designed so. Also, since the parity bits have to be computed using non-sparse operations, the encoding complexity is quadratic with respect to the block length. In fact the only major drawback of LDPC codes compared to turbo codes appears to be their greater encoding complexity. As such, a considerable amount of research has been conducted to take care of the encoding complexity during construction of LDPC codes [19]-[23]. The most common idea in these works is to construct a sparse parity-check matrix $H$ with an efficient encoding format, namely a triangular or almost triangular parity-check matrix. For example in [21] the codeword $c$ is decomposed as $c=(p, d)$, where $p$ and $d$ contain the parity and information bits respectively. Accordingly $H$ is decomposed into $H=\left[H^{p}, H^{d}\right]$. So we have

$$
\left[H^{p}, H^{d}\right] c^{T}=\left[H^{p}, H^{d}\right]\left[\begin{array}{l}
p \\
d
\end{array}\right]=0
$$

Then it was found empirically that the following $m \times m$ square matrix is a good choice for $H^{p}$ :

$$
H^{p}=\left(\begin{array}{cccc}
1 & & & 0 \\
1 & 1 & & \\
& \ddots & \ddots & \\
0 & & 1 & 1
\end{array}\right)
$$


Also the matrix $H^{d}$ is generated based on constructing a random graph that does not contain cycles of length 4 . Now it's easy to see from (2.1) and (2.2) that the parity-check bits can be obtained by:

$$
p_{1}=\sum_{j=1}^{n-m} h_{1, j}^{d} d_{j}(\bmod 2), \text { and } p_{i}=p_{i-1}+\sum_{j=1}^{n-m} h_{i, j}^{d} d_{j}(\bmod 2), i>2
$$

Since $H^{d}$ is designated to be sparse, the computation and storage requirements in the encoder are reduced. So by using this approach we achieve a linear-time encoding but we may experience some loss in the performance in comparison with the performance of the parity-check matrices that have been designed without any constraint for linear-time encoding complexity.

Another interesting result on encoding of LDPC codes is given in [23] where it is theoretically proved that the encoding of optimized LDPC codes can be performed in linear time; in fact, we can generate a random parity-check matrix with an optimized irregular degree sequence pair and an (almost) upper triangular structure which corresponds to a linear-time encoding.

The PEG algorithm can be used to construct LDPC codes with (almost) triangular structure for $H$ meanwhile having good girth properties and with optimum irregular degree sequence [16]. It is worth reminding that, although in PEG algorithm we can just have arbitrary symbol node degree sequences and do not have complete control on the degree of check nodes, the algorithm makes the check degree sequence as uniform as possible which is proved to be optimum in some weak sense [17], [18]. Let's consider a parity-check matrix $H=\left[\not^{p}, H^{d}\right]$ with upper triangular structure. The $m \times m$ component $H^{p}=\left\{h_{i, j}^{p}\right\}$ of the parity-check matrix can be written as: 


$$
H^{p}=\left(\begin{array}{cccc}
1 & h_{1,2}^{p} & \cdots & h_{1, m}^{p} \\
0 & 1 & h_{2,3}^{p} & \vdots \\
\vdots & \ddots & \ddots & h_{m-1, m}^{p} \\
0 & \cdots & 0 & 1
\end{array}\right),
$$

and the parity bits are computed recursively by

$$
p_{i}=\sum_{j=i+1}^{m} h_{i, j}^{p} p_{j}+\sum_{j=1}^{n-m} h_{i, j}^{d} d_{j}(\bmod 2),
$$

from $i=m$ to $i=1$.

Let us decompose the symbol node set $V_{s}$ into the first $m$ symbol nodes (parity bits) and the other $n-m$ symbol nodes (information bits), called the redundant subset $V_{s}^{p}$ and the information subset $V_{s}^{d}$, respectively. Then the PEG algorithm is used to establish the edges of symbol nodes while maintaining the special pattern in (2.4). The procedure of establishing the edges of information subset $V_{s}^{d}$ is the same as before (Deterministic PEG algorithm of page 21). For the edges of $V_{s}^{p}$ the following modified PEG algorithm can be used [16]:

\section{Deterministic PEG Algorithm for Establishing the Edges of $V_{s}^{p}$ :}

For $j=0$ to $m-1$ do

begin

$$
\text { for } k=0 \text { to } d_{s_{j}}-1 \text { do }
$$

\section{begin}

$$
\begin{aligned}
& \text { if } k=0 \\
& E_{s_{j}}^{k} \leftarrow \operatorname{edge}\left(c_{j}, s_{j}\right) \text {, where } E_{s_{j}}^{0} \text { is the first edge incident to } s_{j} \text {. This edge corre- } \\
& \text { sponds to the ' } 1 \text { ' in the diagonal line of matrix } H^{p} \text {. } \\
& \text { else }
\end{aligned}
$$


Expanding a tree from symbol node $s_{j}$ up to depth $l$ under the current graph setting $E_{s_{0}} \cup E_{s_{1}} \cup \ldots \cup E_{s_{j-1}}$, such that $\overline{\mathcal{N}_{s_{j}}^{l}} \cap\left\{c_{0}, c_{1}, \ldots, c_{j-1}\right\} \neq \varnothing$ but $\overline{\mathcal{N}_{s_{j}}^{l+1}} \cap\left\{c_{0}\right.$, $\left.c_{1}, \ldots, c_{j-1}\right\}=\varnothing$, or the cardinality of $\mathcal{N}_{s_{j}}^{l}$ stops increasing. Then $E_{s_{j}}^{k} \leftarrow \operatorname{edge}\left(c_{i}\right.$, $s_{j}$ ), where $E_{s_{j}}^{k}$ is the $k$-th edge incident to $s_{j}$ and $c_{i}$ is one check node picked from the set $\overline{\mathcal{N}_{s_{j}}^{l}} \cap\left\{c_{0}, c_{1}, \ldots, c_{j-1}\right\}$ having the lowest check node degree. If they are multiple choices for $c_{i}$, we select the one with smallest subscript.

end

end

\section{2-2 Decoding of LDPC Codes}

\section{2-2-1 MAP decoder}

Decoding is a decision-making process: Based on the sequence of channel outputs, the decoder tries to figure out which sequence of information bits has been generated at the information source. The goal of the decoder is to minimize the probability of decoding error.

Let $C$ be a binary $(n, k)$ linear block code. Suppose that the codewords $\left\{c_{i}\right\}$ are transmitted according to some probability mass function $p_{C}\left(c_{i}\right)$, while the received codewords arrive at the decoder according to the probability density function $(p d f) f_{R}(r)$. The probability $p c(c \mid r)$ that codeword $c$ is transmitted conditioned on the receipt of word $r$ can be derived from the detailed characteristics of the communication system and channel (e.g., transmitted power level, channel noise level, modulation format). The maximum a posteriori $(M A P)$ decoder finds the codeword $c_{i}$ that maximizes $p_{c}(c \mid r)$. 
Using Bayes' rule, $p_{c}(c \mid r)$ can be related to $f_{R}(r \mid c)$ as follows.

$$
p_{c}(c \mid r)=\frac{p_{C}(c) f_{R}(r \mid c)}{f_{R}(r)}
$$

Computation of $a$ posteriori probabilities requires knowledge of a priori probabilities $p_{C}(c)$ and the conditional probability density functions $f_{R}(r \mid c)$ for all possible transmitted signals. In many cases, the probability of transmission of different codewords can be assumed to be uniformly distributed. Consequently, the maximum a posteriori decoding is equivalent to finding the codeword that maximizes likelihood function $f_{R}(r \mid c)$. The decoder that maximizes $f_{R}(r \mid c)$ is called maximum-likelihood $(M L)$ decoder.

For the binary input additive white Gaussian noise (BLAWGN) channel with noise power spectral density $\frac{N_{0}}{2}$ and BPSK modulation, we can write

$$
f_{R}(r \mid c)=\frac{1}{\sqrt{\left(\pi N_{o}\right)^{n}}} e^{-\frac{\|r-x\|^{2}}{N_{o}}}
$$

in which $x=\left(x_{1}, x_{2}, \ldots, x_{n}\right)$ and $x_{i}=2 c_{i}-1,1 \leq i \leq n$. So, ML decoding is equivalent to finding the codeword $c_{i}$ that minimizes the Euclidean distance:

$$
\|r-c\|=\sqrt{\sum_{m=1}^{n}\left(r_{m}-x_{m}\right)^{2}} .
$$

Fig. 2.5 illustrates MAP rule for 4-PSK modulation, with a priori probabilities $3 / 16$, $5 / 16,3 / 16$, and $5 / 16$, and $4 / 16,4 / 16,4 / 16$, and $4 / 16$, for the signals in counterclockwise order starting from the first quadrant. As can be seen, MAP rule partitions the plane into a number of asymmetric decision regions when a priori probabilities are non-equal. The 
plane is equally partitioned when a priori probabilities are equal. It is clear that in the latter case, we only need to know the Euclidean distances between the received point and 4-PSK symbols. In the context of coding over BIAWGN channel, MAP rule is applied to an $n$-dimensional space and again similar partitioning of space exists. Visualization of the regions however, is not possible for $n$ greater than 3 .

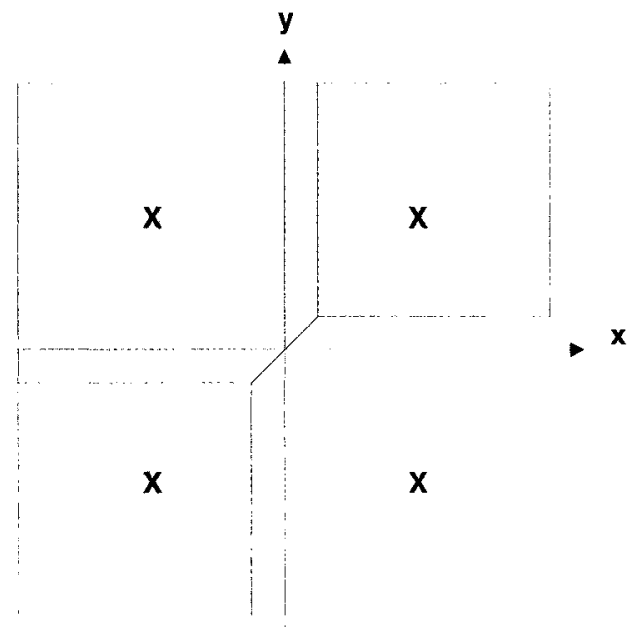

(a)

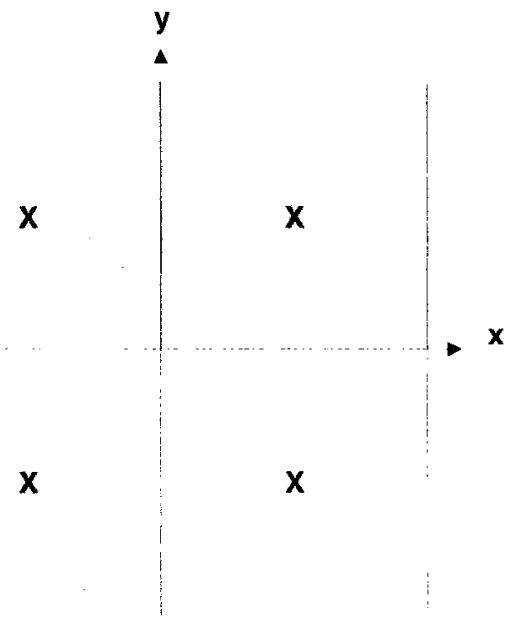

(b)

Fig. 2.5. Decision region of the MAP decoding for a priori probabilities $3 / 16,5 / 16,3 / 16$ and $5 / 16$ (a) and $4 / 16,4 / 16,4 / 16$, and $4 / 16$ (b). Note that (b) also represents ML decoding.

As it was shown, MAP and ML decision rules are equivalent if the condition that all transmitted codewords are equi-probable is met. In general case, however, the $\mathrm{ML}$ decision decoding does not necessarily minimize probability of decoding error, since the distribution of transmitted codewords can not be guaranteed to be uniform. For practical applications, where distribution of transmitted codewords is often unknown, the ML decision rule is often used. This is not a limiting drawback since the goal of possible source encoding is to bring the distribution of transmitted codewords as close to a uniform distribution as possible. 
It is well known that if we allow the block length of LDPC codes to become sufficiently large, they can achieve capacity over a given binary-input memoryless outputsymmetric channel, under ML decoding [24]. However, the decoding complexity of ML decoding of a binary block code increases exponentially with the length of the code and as such, achieving a performance close to the channel capacity by ML decoding of LDPC codes is prohibitively expensive, and practically impossible.

As it was mentioned earlier, for transmission problem we are looking for some codes which allow the transmission of information close to capacity at low probability of error, and can be decoded with an acceptable complexity. For this purpose, as it can be seen from the proof of Shannon's channel coding theorem in [25], which uses itself a suboptimal jointly typical decoder, we are not restricted to ML decoding. Iterative decoding of linear codes has emerged as a promising approach with relatively low complexity and capability of approaching the Shannon limit [19], [26].

\section{2-2-2 Iterative Decoding Algorithms}

Iterative decoding algorithms, also called "message-passing algorithms", operate by sending messages along the edges of a Tanner graph for a number of iterations in both directions. Since the number of edges of Tanner graph is linear in the length of code it follows that for a fixed degree distribution, the decoding complexity per iteration grows only linearly in the block length. For a fixed number of iterations, the total decoding complexity is then a linear function of block length.

An iterative decoding algorithm first generates initial messages for symbol nodes from the observation at the output of the channel and the channel characteristics. Then these messages are sent to check nodes through edges of the graph (Fig. 2.6), and after 
this, iterations start. Each iteration consists of two steps: 1) Check nodes process the information received from the symbol nodes and pass new messages to symbol nodes. These messages reflect the estimate of check nodes from the value and reliability of symbol nodes (Fig. 2.7(a)). 2) symbol nodes process the messages received from check nodes and send back updated messages (information) about their value and the associated reliability to check nodes (Fig. 2.7(b)). In each iteration, hard decision on the value of each bit is made at symbol nodes and the algorithm stops if the hard decision assignment for bits satisfies all the check constraints, or a maximum number of iterations is reached. Usually the message that is sent from a symbol node $s$ or a check node $c$ along an edge $e$, does not depend on the message previously received along edge $e$. Excluding the incoming message along edge $e$, in computing the outgoing message along edge $e$, guarantees that only extrinsic information is passed along and is an important property of good iterative decoders [8]. This property is illustrated on Fig. 2.7; in Fig. 2.7(a) the outgoing message sent from check node $\mathbf{c}$ to symbol node $\mathbf{s}_{d_{c}}, \Psi_{c}\left(\mathbf{m}_{1}, \mathbf{m}_{2}, \ldots, \mathbf{m}_{d_{c}-1}\right)$, is just a function of the incoming messages from all other symbol nodes connected to $\mathbf{c}, \mathbf{m}_{1}, \mathbf{m}_{2}, \ldots, \mathbf{m}_{d_{c}-1}$. Also in Fig. 2.7(b) the outgoing message sent from check node $s$ to symbol node $\mathbf{c}_{\boldsymbol{d}_{\boldsymbol{s}}}$, $\Psi_{s}\left(\mathbf{m}_{0}, \mathbf{m}_{1}, \mathbf{m}_{2}, \ldots, \mathbf{m}_{d_{s-1}}\right)$, is a function of the initial message $\mathbf{m}_{0}$ and the incoming messages from other symbol nodes, $\mathbf{m}_{1}, \mathbf{m}_{2}, \ldots, \mathbf{m}_{d_{s}-1}$. 


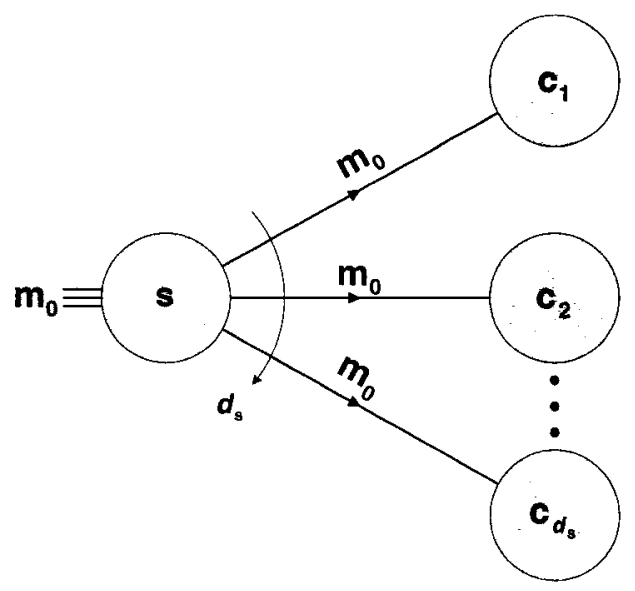

Fig. 2.6. Before going through iterations, initial messages are sent to check nodes
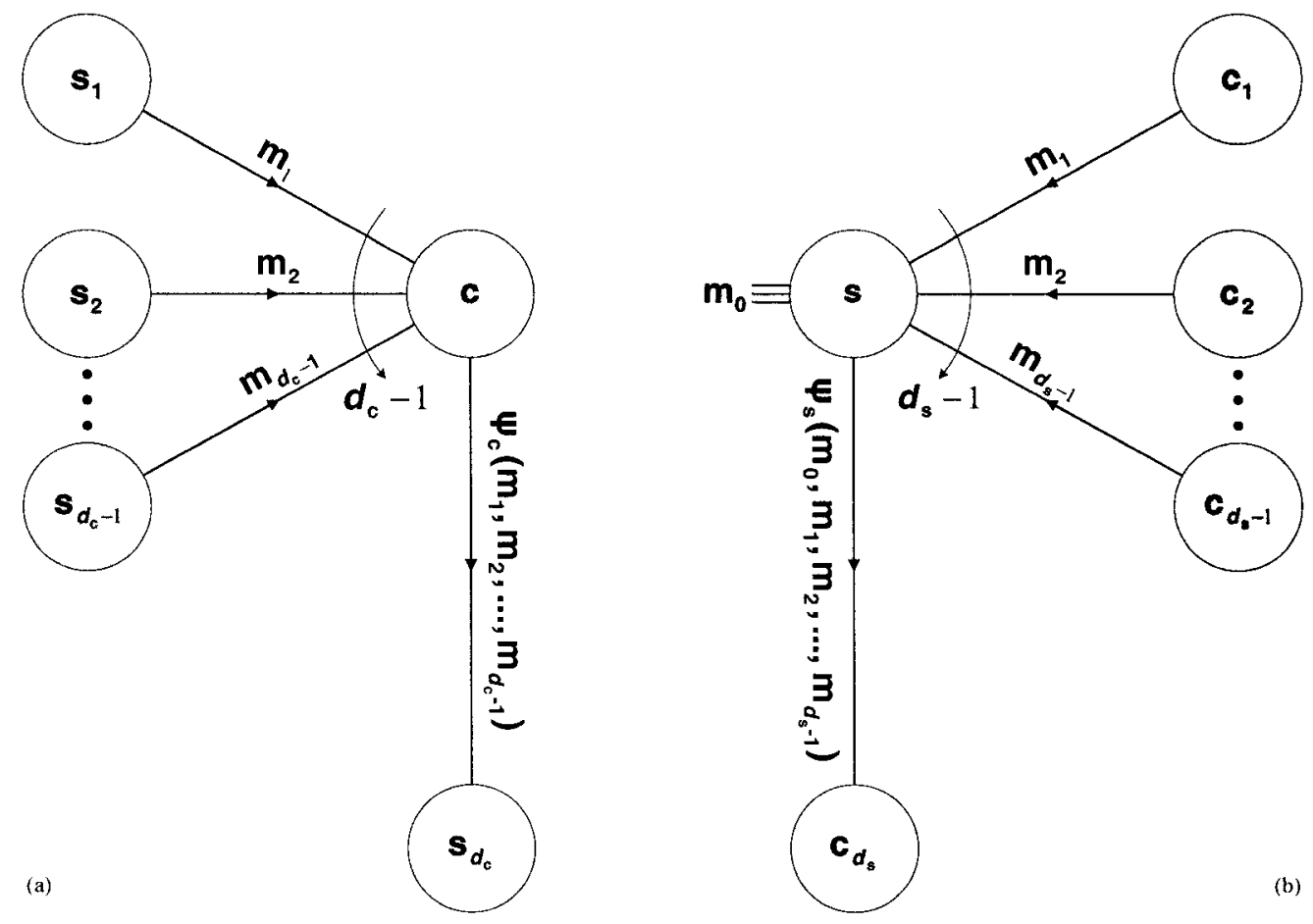

Fig. 2.7. (a) Check nodes $\mathbf{c}$ sending a new message to symbol node $\mathbf{s}_{\mathrm{d}_{\mathrm{c}}}$. (b) Symbol node $\mathbf{s}$ sending back updated message to check node $\mathbf{c}_{\mathrm{d}_{\mathrm{s}}}$. 
Among iterative decoding algorithms, BP algorithm, also known as SumProduct, is the most famous algorithm and has the best performance. Here we describe this iterative decoding algorithm over AWGN channel.

\section{2-2-2-1 Belief Propagation Algorithm}

The BP algorithm was first introduced by Gallager in his thesis to decode LDPC codes [1]. Later, Tanner [2] generalized Gallager's LDPC codes to codes on Tanner graphs, developed BP for decoding these codes, and proved their convergence on cyclefree graphs. Wiberg [4] proved in his thesis that if the Tanner graph is cycle-free, BP algorithm computes the exact marginal posterior probabilities. Fortunately, for graphs containing cycles, as is the case for LDPC codes, BP algorithm still delivers impressive results. This observation along with the fact that BP is practically implementable, have instigated a lot of research in both theoretical and practical aspects of this algorithm.

Assume that each bit $c_{i}$ of a codeword $c=\left(c_{1}, c_{2}, \ldots, c_{n}\right)$ is mapped into $x_{i}$ according to $x_{i}=2 c_{i}-1$. Then $\boldsymbol{x}=\left(x_{1}, x_{2}, \ldots, x_{n}\right)$ is transmitted over a binary input additive white Gaussian noise (AWGN) channel. At the output of the channel, we have $r=x+n$, where $\boldsymbol{n}$ is a Gaussian random vector independent of $\boldsymbol{x}$, whose elements are independent zeromean Gaussian random variables with variance $\sigma^{2}=N_{0} /\left(2 R E_{b}\right)$, in which $R$ is the rate of the code, and $E_{b}$ and $\frac{N_{0}}{2}$ are the average energy per information bit and the power spectral density of AWGN, respectively. Derivation of symbol node and check node operations is based on the assumption that in the $l$ th iteration of the BP algorithm, there are no cycles of length $2 l$ or less in Tanner graph of the code [8]. It is convenient to use loglikelihood ratios (LLRs) for messages: i.e., $m_{s \rightarrow c}=\log \frac{p(y \mid x=1)}{p(y \mid x=-1)}$ is used as the out- 
put message of a symbol node $s$ passed to check node $c$, where $x$ is the bit value of the node $s$ and $y$ is a random variable describing all information incorporated into this message. More precisely, at each iteration $l, y$ denotes all information available to the node $s$ obtained from edges other than the one carrying $m_{s \rightarrow c}$, which in fact carry the information from the symbol nodes of a tree with depth $l$ rooted at node $s$. Likewise, the output message of a check node $c$ passed to a symbol node $s$ is defined as $m_{c \rightarrow s}=\log \frac{p\left(y^{\prime} \mid x=1\right)}{p\left(y^{\prime} \mid x=-1\right)}$ where $x$ is the bit value of the symbol node $s$ and $y^{\prime}$ is a random variable describing all information incorporated into this message. This information has been obtained from edges other than the one carrying $m_{c \rightarrow s}$, and in fact is the information from the symbol nodes of a tree with depth $l$ rooted at node $c$.

Obviously at the beginning of algorithm the received values from channel and the channel characteristics are the only available information to the symbol nodes. So the algorithm starts with sending the following initial messages from symbol nodes to check nodes:

$$
m_{s \rightarrow c}=m_{i}(0)=\frac{2}{\sigma^{2}} r_{i}
$$

Then by performing the following operations iteratively, BP algorithm tries to estimate the transmitted codeword. The derivation of the check node and symbol node operations can be found in [8].

Check node step:

$$
m_{c \rightarrow s}=\log \frac{1+\prod_{k \in N(c) \|_{s}} \tanh \left(0.5 m_{k \rightarrow c}\right)}{1-\prod_{k \in N(c) \|_{s}} \tanh \left(0.5 m_{k \rightarrow c}\right)} .
$$


where $N(c) \backslash s$ is the set of all neighboring symbol nodes of $c$ other than $s$.

Symbol node step:

$$
m_{s \rightarrow c}=m_{i}^{(0)}+\sum_{k \in N(s) / c} m_{k \rightarrow s}
$$

where $N(s) \backslash c$ is the set of all neighboring check nodes of $s$ other than $c$.

Hard decision and stopping criteria (at bit node s):

$$
\begin{gathered}
w_{s}=m_{i}^{(0)}+\sum_{k \in N(s)} m_{k \rightarrow s}, \\
\hat{s}=\frac{1+\operatorname{sgn}\left(w_{s}\right)}{2} .
\end{gathered}
$$

The algorithm stops if the estimated hard-decision assignment, consisting of $\hat{s}$ 's, is a codeword, or a maximum number of iterations is reached.

\section{2-3- Performance of LDPC Codes under Belief-Propagation}

\section{Algorithm}

In this section we compare the performance of regular and irregular LDPC codes obtained by the random Poisson construction and PEG construction, under BP algorithm. Although the performance of some of these codes under BP algorithm, has been reported in other references, all of our comparisons are made based on our simulation results to make sure that the comparisons are fair in terms of using the same simulation program and the same confidence interval for simulations. In fact, if there are minor differences between our simulation results and other reported results, they are due to slight differences between the simulation programs and confidence intervals. Also for constructing LDPC codes based on the PEG algorithm, we have used the deterministic versions of 
PEG algorithms that were described in previous section and thus the generated codes may have small differences in girth distribution (and maybe small differences in performance) with the codes that have been generated in [16] by using a non-deterministic version of PEG algorithm. In all of our simulations, for each signal to noise ratio enough noisy codewords are simulated to generate at least 200 codeword errors.

\section{2-3-1- Regular Codes}

The first comparison is made between two regular LDPC codes of rate- $1 / 2$ and length $n=504$. One of these codes is the MacKay's $(3,6)$-regular LDPC code that has been generated using Poisson Construction by avoiding cycles of length four [27], and is one of the best codes with these parameters. The other code has a PEG Tanner graph of 504 symbol nodes and 252 check nodes in which all the symbol nodes have degree 3 and almost all the check nodes are of degree 6 , except for 8 check nodes of degree 7 and 8 with degree 5. In the PEG Tanner graph each symbol node has a local girth of 8 while in the MacKay's code, $63 \%$ of the symbol nodes have a local girth of 6 and $37 \%$ of 8 . Obviously the PEG Tanner graph has a superior girth histogram. Fig. 2.8 shows the curves of bit-error-rate $(B E R)$ and word-error-rate (WER) for these two codes, with the maximum number of iterations equal to 80, over an additive white Gaussian noise (AWGN) channel. The LDPC code generated by PEG algorithm is always slightly better than MacKay's

code. At a WER of $5 \times 10^{-5}$, the LDPC code generated by PEG algorithm outperforms MacKay's code by about $0.2 \mathrm{~dB}$. Since these two codes have the same rate and a very close degree distribution, they have essentially the same decoding complexity and thus this gain is achieved without any increase in the decoding complexity. The significance 
of this difference would be clearer if we note that MacKay's codes are among strong codes for short and medium block length.

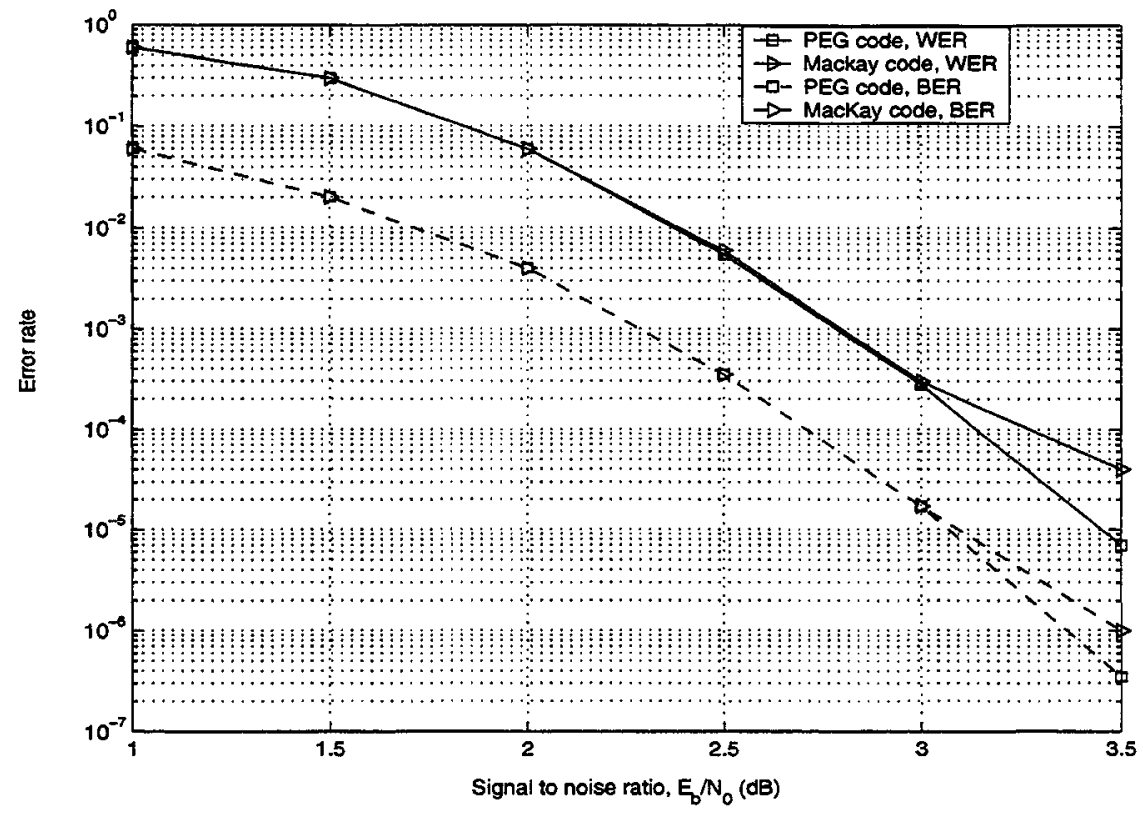

Fig. 2.8. BER and WER of a PEG-constructed code and MacKay's code with parameters $n=504$,

$$
m=252, d_{s}=3, d_{c}=6
$$

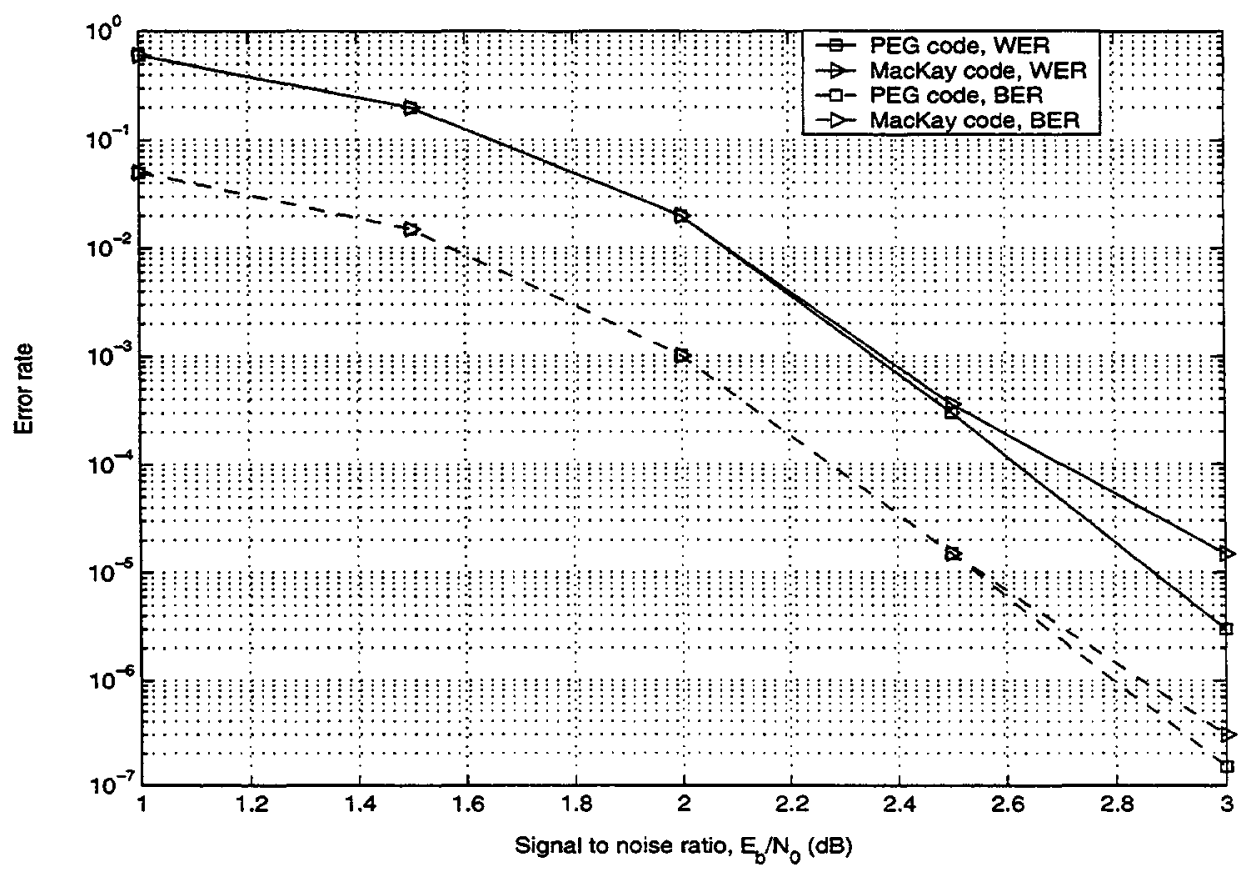

Fig. 2.9. BER and WER of a PEG Tanner-graph code and MacKay's code with parameters $n=1008$,

$$
m=504, d_{s}=3, d_{c}=6 .
$$


For the second comparison we consider two LDPC codes of rate-1/2 and length $n=1008$. The first code is the MacKay's $(3,6)$-regular LDPC code of length 1008 which has been produced using Poisson construction by avoiding cycles of length four [27], and again is one of the best codes with these parameters. The second code has a PEGconstructed Tanner graph with 1008 symbol nodes of degree 3, 30 check nodes of degree 7, 30 check nodes of degree 5, and 444 check nodes of degree 6. In the PEG-constructed Tanner graph, $7 \%$ of symbol node have a local girth of 8 and $93 \%$ of 10 , while in the MacKay's code, $39.5 \%$ of the symbol nodes have a local girth of 6 and $60.3 \%$ of 8 . Fig. 2.9 compares the BER and WER for these two $(1008,504)$ codes after 80 iterations. Again, we see that the LDPC code based on PEG Tanner graph is slightly better than MacKay's code. Also note that these two codes have the same rate and very similar degree distributions and thus this improvement is obtained without any increase in decoding complexity.

\section{2-3-2- Irregular Codes}

In [19], [28], and [29] it was shown that properly designed irregular LDPC codes can outperform regular ones. Having large girth is only one condition for an irregular LDPC code to have a good performance; design of good irregular degree sequence for symbol nodes and check nodes is equally or even more important. For very long block codes, a concentration theorem has been proved in [8] which states that at very large block lengths, the performance of an ensemble of LDPC codes is concentrated around its mean which itself is the performance of a cycle-free graph with the same parameters. This allows us to find optimum degree sequences by density evolution techniques. Density evolution is basically a technique for obtaining, through deterministic algorithms, expres- 
sions for the probability density functions of the messages which are passed in each iteration of an iterative decoding algorithm, provided that the Tanner graph of the code is effectively cycle free. The irregular LDPC codes which have the optimum degree sequences, devised by using density evolution techniques, and have been constructed based on random Poisson construction, give performances extremely close to Shannon limit on AWGN channel at large block lengths.

However for short LDPC codes the cycle-free assumption is no longer valid and thus the density evolution technique cannot be used to obtain optimum degree sequences. In [9] the average of girth distribution is used as an efficient selection criterion to find good codes among random graphs of short block lengths. In [16], an empirical Monte-Carlo approach has been introduced which uses a variant of the "downhill simplex" optimization technique [30], [31], to design symbol node-degree distribution for short codes with fewer than a thousand bits. Here, like regular codes, the degree of check nodes is made as uniform as possible [17], [18]. Also, the cost function of the optimization problem is considered to be the WER at the largest $E_{b} / N_{o}$ in which none of the simulated WER values, corresponding to different PEG graphs, is zero. The codes with short block length, and the above degree sequences, that have been constructed based on PEG construction are the best ones to date in terms of WER. Interestingly, the performance of theses codes is more or less the same as the performances of the PEG-constructed LDPC codes that have the best degree sequences obtained by density evolution in [26].

For example, for a rate- $1 / 2$ code with $n=504, m=252$, the optimized degree distribution is $\lambda(x)=0.47532 x^{2}+0.279537 x^{3}+0.0348672 x^{4}+0.108891 x^{5}+0.101385 x^{15}$ [16]. The Tanner graph of this code has $239,141,18,55$, and 51 symbol nodes with de- 
grees $2,3,4,5$, and 15 , respectively. Also there are 22,205 , and 25 check nodes with degrees 7,8 , and 9, respectively. In Fig. 2.10 the performance of the PEG constructed LDPC code with these characteristics is presented. We observe that this code outperforms MacKay's rate-1/2 regular code of the same length by about 0.3 to $0.4 \mathrm{~dB}$ in all range of $E_{b} / N_{0}$ with 80 iterations. Also in Fig. 2.10, the performance of an irregular LDPC with the same symbol-degree distribution, that has been constructed based on Poisson construction by avoiding cycles of length 4 , is presented. In this code, there are 249 and 3 check nodes with degrees 7 and 8 respectively. This code exhibits a very early error floor. This clearly shows that at short block lengths, using PEG construction for constructing LDPC codes has a significant advantage over the random Poisson construction.

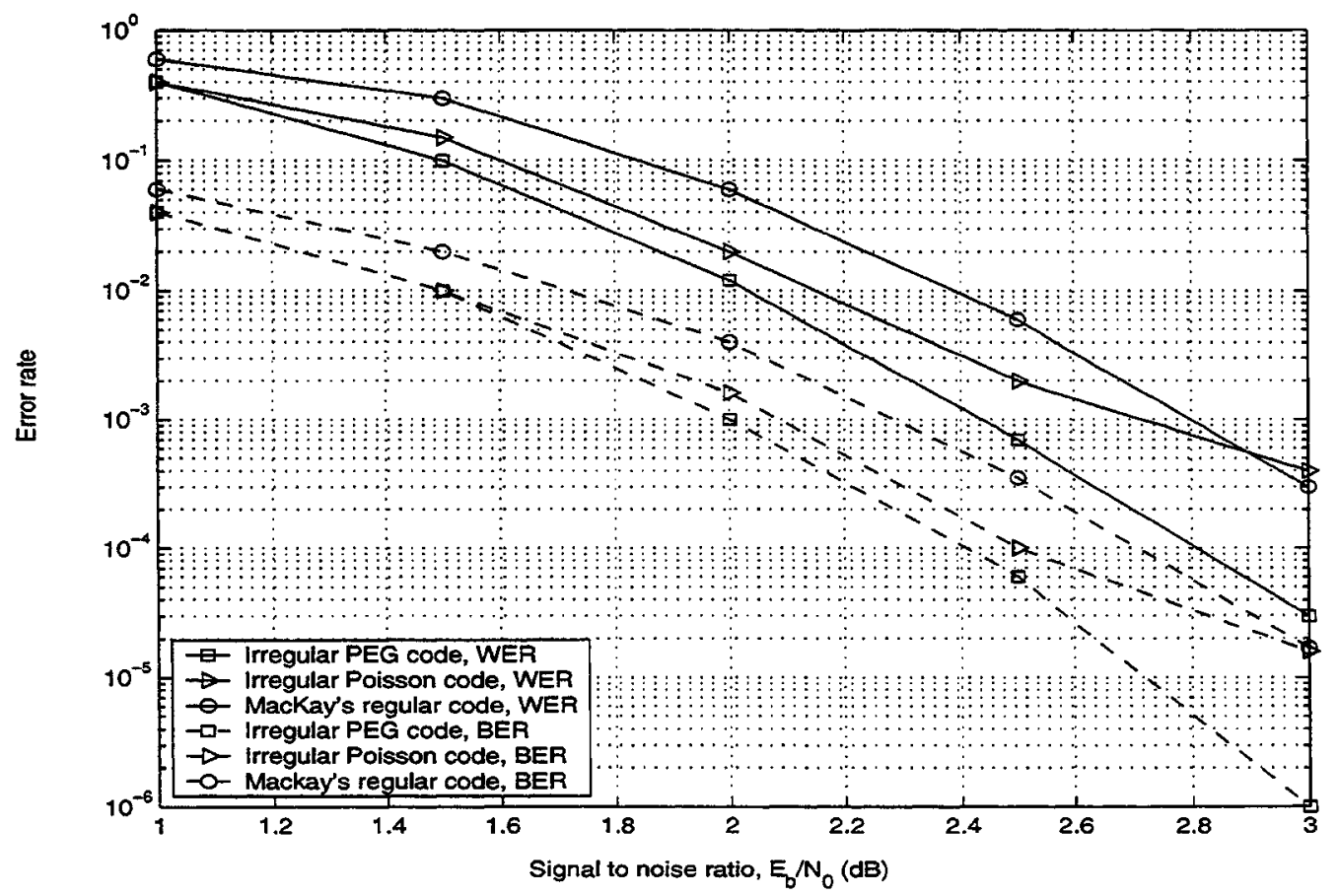

Fig. 2.10. BER and WER of an irregular PEG Tanner-graph, irregular random-graph code, and MacKay's regular code with parameters $n=504, m=252$

The same degree distribution can be used to construct an LDPC code with $n=1008$, $m=504$. This code has $479,282,35,110$, and 102 symbol nodes with degrees $2,3,4,5$, 
and 15, respectively. Also it has 31, 450, and 23 check nodes with degrees 7, 8, and 9, respectively. We have compared the performance of this code with that of an irregular LDPC with the same symbol-degree distribution, that has been constructed based on Poisson construction by avoiding cycles of length 4 . This code has 496 check nodes of degree 8 and 8 check nodes of degree 7 . Simulation results for these two codes are presented in Fig. 2.11. Again we observe that the code constructed by PEG construction outperforms MacKay's $(3,6)$-regular code of rate-1/2 by up to $0.3 \mathrm{~dB}$ and also works considerably better than the similar irregular Poisson-constructed code which shows an early error floor.

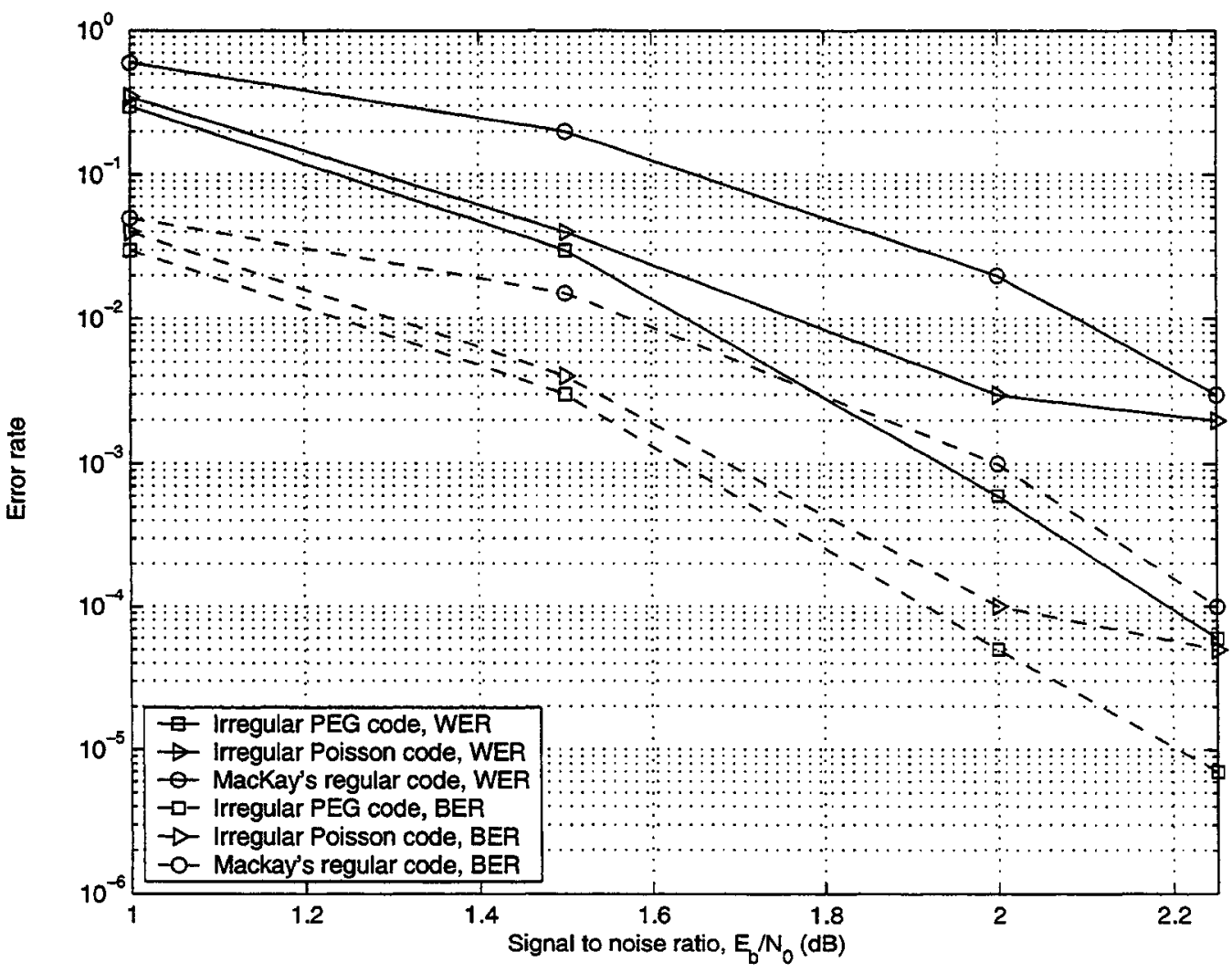

Fig. 2.11. BER and WER of an irregular PEG Tanner-graph, irregular random-graph code, and MacKay's regular code with parameters $n=1008, m=504$ 


\section{2-3-3- Linear-Time Encodable Irregular LDPC Codes}

As it was mentioned in the first section, linear-time encoding property can be naturally combined with the PEG construction and as we will see in the following, this comes without any degradation in the performance of the code. Let us consider an irregular PEG code whose parity-check matrix is forced to be an upper-triangular matrix and has been constructed by deterministic modified-PEG algorithm described in subsection 2-1-3, with parameters $n=1008, k=504$, and based on the symbol degree distribution function $\lambda(x)=0.47532 x^{2}+0.279537 x^{3}+0.0348672 x^{4}+0.108891 x^{5}+0.101385 x^{15}$. Based on this degree distribution and because we want the parity-check matrix to have an upper triangular form, the Tanner graph of this LDPC code has one symbol node with degree 1 and the remaining symbol nodes will be of degree $2,3,4,5$, and 15 with $478,282,35$, 110 , and 102 symbols with each degree, respectively. Also the check nodes have degrees 7,8 , and 9 with 31,451 , and 22 check nodes with each degree, respectively. Fig. 2.12 compares the performance of this code with the PEG-constructed code of Fig. 2.11 which has the same characteristics except that it does not have an upper-triangular parity-check matrix. As we see, the two irregular codes have essentially the same performance. So linear-time-encoding property can be achieved without noticeable degradation in the performance of the code, under PEG construction. 


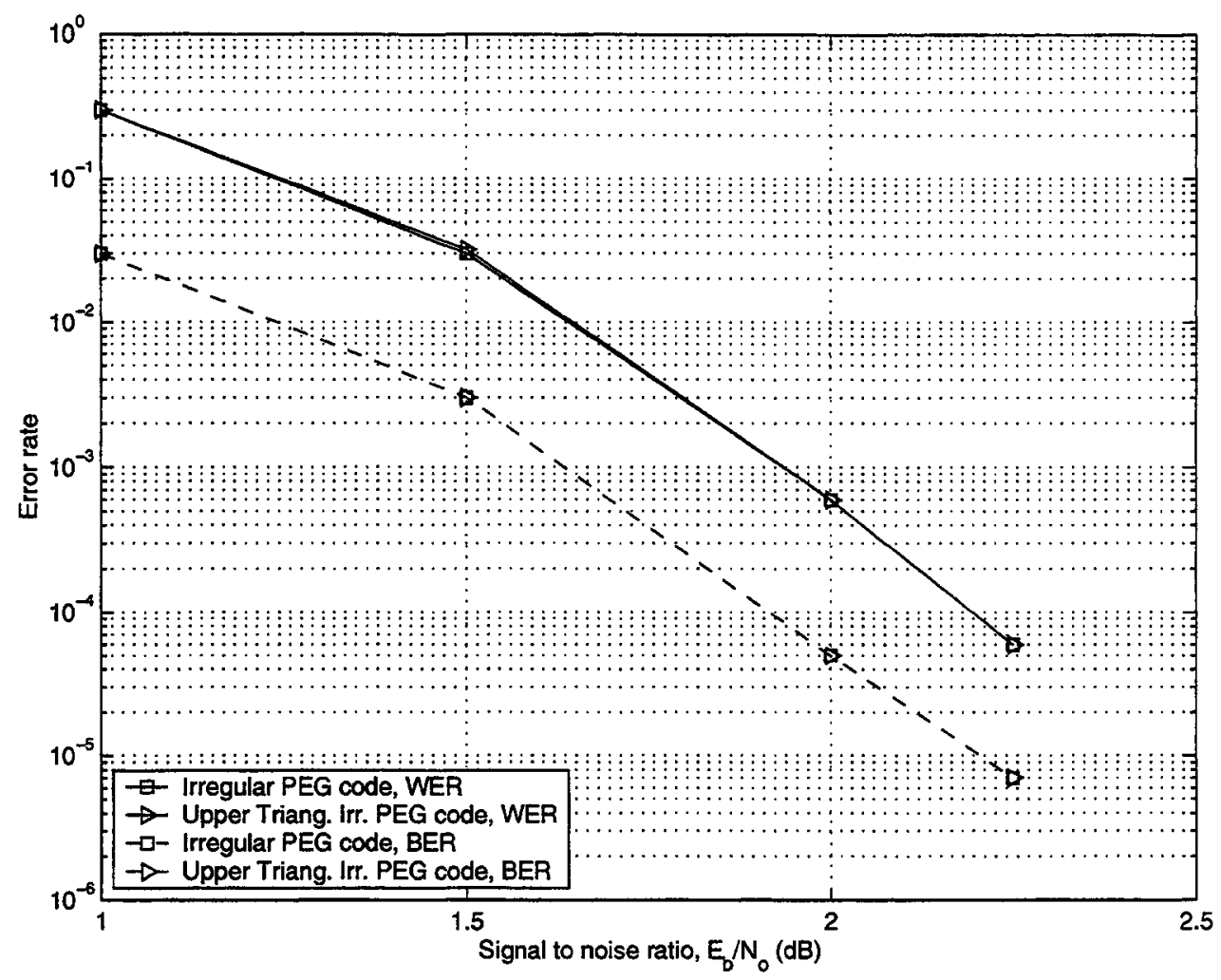

Fig. 2.12. BER and WER of an irregular PEG Tanner-graph and an irregular upper-triangular PEG code with parameters $n=1008, m=504$ 


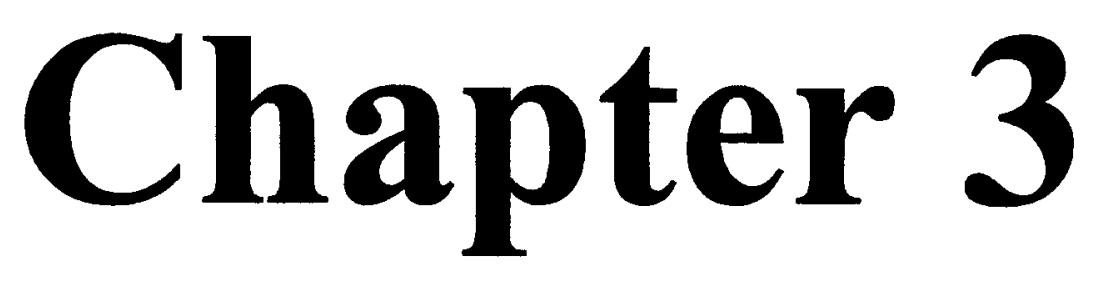

\section{Automatic Repeat-Request}

(ARQ) Protocols 
Error-Correcting codes can be employed on one-way channels to correct errors at the receiver. Such error control schemes are called forward error correction (FEC). In conventional applications of FEC, depending on the channel characteristics and the acceptable error rate of data, a fixed rate coding scheme is used. However, to address different error protection requirements of different data streams or time varying nature of mobile radio channels, more flexible channel coding schemes are desirable. In this regard, automatic repeat-request ( $A R Q$ ) protocols have the potential for adaptation to changes in the transmission medium. In these protocols the transmitted data is encoded for error detection; when an error is detected at the receiver, a retransmission request is sent back to the transmitter through a reliable channel. The transmitter then keeps sending the data until no error is detected at the receiver.

In this chapter we describe basic principles of different types of ARQ protocols. First pure ARQ protocols are explained. Then the type-I hybrid-ARQ protocol is examined and finally we describe the type-II hybrid-ARQ protocol.

\section{3-1 Pure ARQ Protocols}

Consider an application in which the communication channel is error-free except for occasional bursts of noise of short duration. Such noise bursts can be generated by nearby power machinery, electrical storms or single-event upsets in digital hardware. In such situations, a good level of protection can be obtained by using a simple error detecting protocol; the objective is to detect occasional error bursts, discard the affected packets, and request a retransmission. If the retransmission of packet does not begin in the course of noise burst, multiple retransmissions will not be necessary. 
Cyclic redundancy check $(C R C)$ codes are the most frequently used error detecting codes for pure ARQ protocols. These codes are very easy to design and their encoders can be easily implemented using binary shift-registers [32].

\section{3-1-1 Performance of ARQ Protocols}

The performance of an ARQ protocol can be evaluated by two basic parameters; reliability and throughput.

\section{3-1-1-1 Reliability}

In an ARQ system, a decoding error happens when the receiver accepts a packet with undetected error. Let $P(E)$ denote the probability that the receiver accepts a packet containing errors. Obviously, for a reliable ARQ system $P(E)$ should be very small. Therefore the reliability of an ARQ protocol is measured by its accepted packet error probability $P(E)$. For a pure ARQ protocol the accepted packet error probability can be computed easily. We suppose that the feedback channel is error free and also having undetected errors in successive retransmissions is independent and completely determined by the employed code. Let the error detecting code $C$ have probability $P_{e}$ of undetected error and probability $P_{r}$ of detected error (probability of a retransmission request). A packet is erroneously accepted if, on any transmission attempt, it arrives at the receiver containing an undetectable error pattern. Thus we have:

$$
\begin{aligned}
P(E) & =P_{e}+P_{r} P_{e}+P_{r}^{2} P_{e}+P_{r}^{3} P_{e}+\cdots+P_{r}^{k} P_{e}+\cdots \\
& =P_{e} \sum_{k=0}^{\infty} P_{r}^{k}=\frac{P_{e}}{1-P_{r}} .
\end{aligned}
$$


Equation (3.1) shows that the packet error probability increases with the probability of a retransmission request. In fact each additional retransmission provides another opportunity for the receiver to make a mistake.

\section{3-1-1-2 Throughput}

The throughput of an ARQ protocol is defined as the average number of encoded data bits successfully accepted by the receiver in the time that is required to send a single bit [32]. The throughput is a function of two parameters: 1- The number of times that a packet is retransmitted before it is accepted by the receiver. 2- The retransmission request protocol that the transmitter and receiver choose for handling retransmission requests. In choosing a retransmission request protocol the designer must achieve a proper balance between the complexity and the throughput performance of the resulting system. There are three basic retransmission request protocols; the stop-and-wait $A R Q$, the goback-N $A R Q$, and the selective-repeat $A R Q$.

In a stop-and-wait ARQ protocol the transmitter sends out a packet to the receiver and waits for an acknowledgment from the receiver as shown in Fig. 3.1. The receiver processes the packet and if the packet is deemed error-free it responds by sending $a$ positive acknowledgment $(A C K)$; the transmitter sends the next packet. A negative acknowledgment $(N A K)$ from the receiver indicates that the received packet contains a detectable error; the transmitter resends the packet. Retransmissions continue until an ACK is received by the transmitter. The primary advantage of the stop-and-wait ARQ scheme is that it does not need to buffer the packets at the receiver. As we can see from Fig. 3.1 the transmitter sends packet \#2 after packet \#1 has been accepted by the receiver. However, 
the stop-and-wait ARQ scheme is inherently inefficient because the transmitter is idle while waiting for an acknowledgment from the receiver.

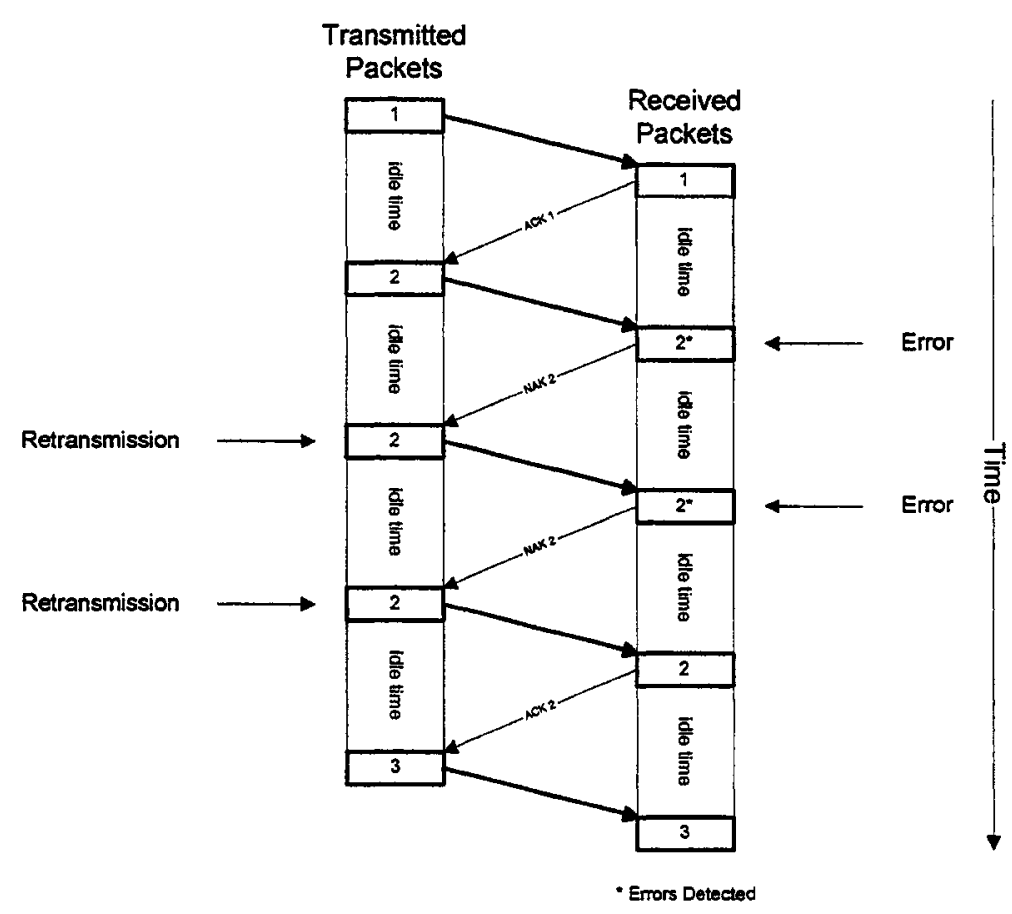

Figure 3.1. Stop-and-wait ARQ

In a go-back-N ARQ protocol the transmitter sends packets continuously. The illustration of the protocol is shown in Fig. 3.2. Here, after sending a packet, the transmitter does not wait for an acknowledgment and begins sending the next packet. It receives the acknowledgment for a packet after a round-trip delay. The round-trip delay is defined as the time it takes for the transmitter to send a packet and receive its acknowledgment. During this time interval, $\mathrm{N}-1$ other packets have also been sent. The term "go-back- $\mathrm{N}$ " comes from the fact that when the transmitter receives a NAK, it must go back up to the packet that is negatively acknowledged and restart retransmission from here (i.e. the transmitter pulls back and resends $\mathrm{N}$ packets). Of course, buffering is necessary at the transmitter for these packets. 


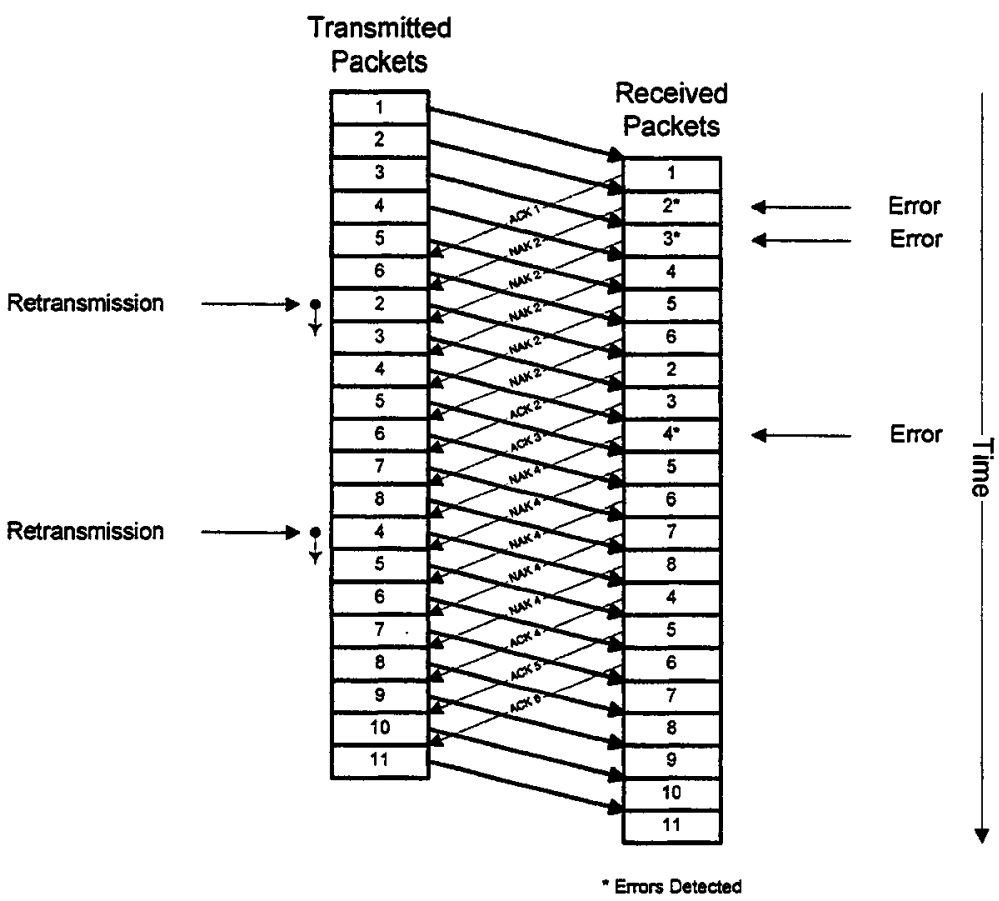

Figure 3.2. Go-back-N with $\mathrm{N}=5$

At the receiver, all the $\mathrm{N}-1$ packets following an erroneously received packet are ignored regardless of whether they are error-free or not. Thus, receiver buffering is not necessary. Compared with stop-and-wait ARQ, the go-back-N ARQ scheme becomes inefficient when the round trip delay is large and the rate of transmitted data is high. This inefficiency is caused by the retransmission of many error-free packets following an erroneously received packet, as well as large buffering requirements. This inefficiency can be removed by using the selective-repeat ARQ scheme.

The illustration of a selective-repeat ARQ protocol is shown in Fig. 3.3. Just as in the go-back-N ARQ protocol, the transmitter sends the packets continuously. However, in this case only those packets that are negatively acknowledged will be resent. It then resumes transmission of new packets from the point at which it stopped. In order to deliver the packets to the user in right order, the receiver needs a buffer to store the error-free received packets that follow an erroneously received packet. 


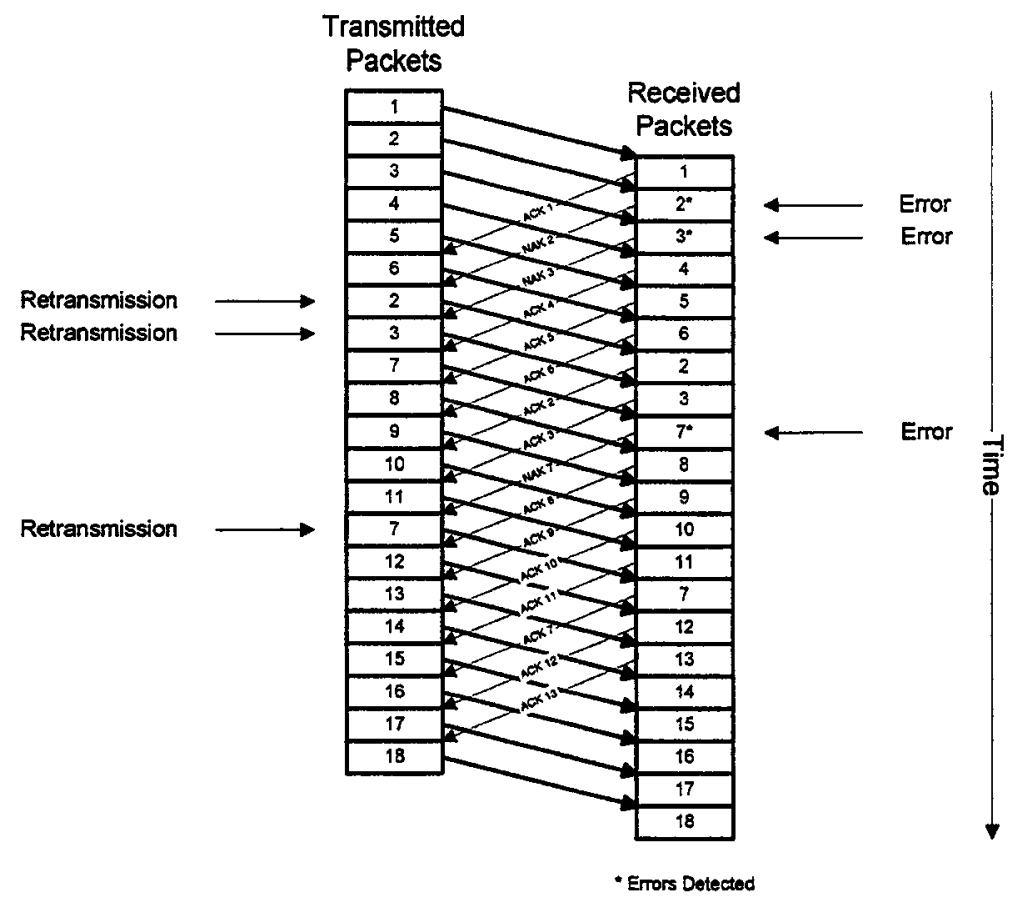

Figure 3.3. Selective-repeat ARQ

After successfully receiving the first negatively acknowledged packet, the receiver puts the error-free received packets in consecutive order until the next error is detected in the received packets. Sufficient receiver buffer is required to avoid buffer overflow and loosing data. As it is intuitively expected, throughput analyses [32] show that the selective-repeat ARQ is the most efficient protocol among the three retransmission protocols. However, it also has the most implementation complexity.

\section{3-2- Type-I Hybrid-ARQ Protocols}

Pure ARQ protocols have a primary drawback; as the channel error rate increases the increased frequency of retransmission requests severely affects the throughput. The hybrid-ARQ protocols overcome this drawback by properly combining FEC and error detection. The FEC portion reduces the frequency of retransmission by correcting the error patterns that occur most frequently on the channel, while the error detection portion de- 
tects the error patterns that happen less frequently (and are most likely to cause decoder errors in FEC system). As a result, a proper combination of FEC and ARQ would both provide throughput similar to that of FEC systems and offer reliability performance typical of ARQ protocols. The FEC can be used in conjunction with any of the three retransmission request protocols. The type-I hybrid-ARQ protocol is the most straightforward hybrid-ARQ protocol. Here we use an $(n, k)$ linear code which is designed for simultaneous error detection and error correction. When a packet error is detected at the receiver, it first tries to correct the errors. If the number of errors is within the error-correcting capability of the code, the receiver corrects them and the decoded message is passed to the data sink. If the error pattern is uncorrectable, the receiver requests a retransmission. The receiver goes through the same procedure for retransmitted packet until the packet is successfully received or decoded.

Since in this scheme, the used code has to both correct a certain number of errors and detect other error patterns, it needs more parity-check bits. This increases the overhead of each transmission and subsequent retransmissions. Consequently, at low error rates its throughput is lower than its corresponding ARQ scheme. Nonetheless, as the channel error rate increases, the throughput of the ARQ scheme falls rapidly and the type-I hybridARQ scheme achieves a higher throughput. If the error correcting capability of the code is sufficiently large, the type-I hybrid-ARQ protocol maintains a significantly higher throughput over a wide range of channel error rates [32].

\section{3-3- Type-II Hybrid-ARQ Protocols}

Type-II hybrid-ARQ protocols have been developed based on the concept of incremental redundancy [33], which implies that the parity check bits for error correction be 
sent to the receiver only when they are needed. In incremental redundancy systems, the transmitter sends additional parity bits to the receiver in response to retransmission requests. The receiver achieves an increased error correction capability by adding these bits to the received packet. The simplest form of incremental redundancy systems is the typeII hybrid-ARQ protocols. Here we describe the original type-II Hybrid-ARQ system [34]. Two linear codes are used in this system; one is a high rate $(n, k)$ error detecting code $C_{1}$, and the other is an invertible systematic $(2 n, n)$ code $C_{2}$ which is designed for simultaneous error correction and error detection. An invertible code is defined to be a code in which the information bits of each codeword can be uniquely determined from the corresponding parity bits. When a $k$-bit message is ready for transmission, it is first encoded using the error-detecting code $C_{1}$ to form an $n$-bit packet $P_{1}$. Then $P_{1}$ is encoded using $C_{2}$. The $C_{1}$ codeword $P_{1}$ is transmitted and the $n$ parity bits (call them $P_{2}$ ) obtained from $C_{2}$ are stored in the retransmission buffer of the transmitter for later use. At the receiver, the received packet corresponding to $P_{I}$ is checked for errors and if errors are detected in it, a NAK is sent back to the transmitter. Upon receiving this NAK the transmitter responds by sending $P_{2}$. Since $\mathrm{C}_{2}$ is invertible, the $n$ bits used to generate $P_{2}$ can be obtained by inverting $P_{2}$. So the receiver generates an inverted version of $P_{2}$ and again uses the decoder of $C_{I}$ to check it for errors. If errors are detected in the inverted version, then a noisy codeword of $C_{2}$ is generated by attaching $P_{2}$ to $P_{1}$. After FEC decoding of this noisy codeword, the decoded message is checked again for errors. If there are still errors, the protocol continues by alternately transmitting $P_{1}$ and $P_{2}$ until no error is detected in one of the three error detection decoding operations. 
The disadvantage of the type-I hybrid-ARQ protocol is that regardless of channel error rate, extra parity-check bits for error correction must be included in each transmission or retransmission. However, when the noise of channel is low, this is not necessary. As we will see in the next chapter, the type-II hybrid-ARQ protocol has the potential to remove this problem by adaptively sending enough parity bits to the receiver. 


\section{Chapter 4}

\section{A Type-II Hybrid-ARQ Protocol}

Based on PEG-Constructed Rate-

Compatible LDPC Codes 


\section{4-1 Introduction}

In the previous chapter we mentioned that type-II hybrid-ARQ protocol takes advantage of both the error correction capability of FEC codes and the rate flexibility of ARQ protocols. The original type-II hybrid-ARQ protocol can be generalized as the following general scheme which is more flexible and has more potential to accommodate different error protection requirements: Initially the transmitter sends $k$ information bits and $r$ CRC bits. In response to subsequent retransmission requests, the transmitter sends parity bits generated by encoding the information and $\mathrm{CRC}$ bits with a systematic code. At the receiver, the newly received parity bits are combined with those previously received to decode the frame with a lower WER. After each NAK, the receiver decodes a stronger, lower rate code, derived from the initial mother code. In fact, here the mother code is punctured in a rate-compatible $(R C)$ way, meaning that the parity bits of each code are embedded in the parity bits of the lower rate codes. With this flexible structure, type-II hybrid ARQ system has the ability to adapt its throughput as channel conditions change. The power of the RC code used in the system is the key parameter concerning the throughput efficiency of type-II hybrid-ARQ scheme. BCH and convolutional codes have been used to create easily implementable RC codes [35], [36], [37], and [38]. They however do not provide near capacity performance. Turbo codes and LDPC codes are the most powerful available FEC schemes and thus using them in hybrid-ARQ systems offers the possibility of achieving the best possible performance.

In this chapter we first present the analyses and equations that govern the type-II hybrid-ARQ protocols. Then a brief review of type-II hybrid-ARQ protocols based on ratecompatible punctured turbo (RCPT) codes and their throughput performance will be pre- 
sented. In the last section we first describe two techniques that can be used to generate RC-LDPC codes and then we explain how a combination of these techniques can be used to efficiently construct a family of RC-LDPC codes. Finally, we show that the irregular RC-LDPC codes constructed based on PEG construction of [16] with upper-triangular parity-check matrices provide a very good throughput performance for type-II hybridARQ protocols at short block lengths while lending themselves to linear-time encodability. We refer to these codes as "linear-time encodable PEG-constructed RC-LDPC codes", or in brief as "PEG-RC-LDPC codes".

\section{4-2 Analysis}

In the following we first analyze the throughput of a type-II hybrid-ARQ protocol and then upper bound the throughput by the capacity curve.

Note that we consider system performance as a function of the symbol signal to noise ratio, $E_{s} / N_{o}$, as opposed to the conventional bit signal to noise ratio, $E_{b} / N_{o}$. For example at $E_{S} / N_{o}=0 \mathrm{~dB}$, if a RCPT-ARQ system, on average, transmits information successfully with adding one parity bit per information bit, then this implies an average $E_{b} / N_{o}$ of $3 \mathrm{~dB}$. So different ARQ systems may be compared assuming a given $E_{s} / N_{o}$. (In a fixed-rate FEC scheme, given $E_{b} / N_{o}, E_{s} / N_{o}$ is fixed and is related to $E_{b} / N_{o}$ by $E_{s} / N_{o}=R \times\left(E_{b} / N_{o}\right)$. However in an ARQ scheme, rate is not fixed and therefore it is much easier to work with $E_{s} / N_{o}$ as opposed to $\left.E_{b} N_{o}\right)$. 


\section{4-2-1 Throughput Analysis}

Let $k$ be the length of an information frame and $N_{a v}$ be the average total number of bits that must be transmitted in order for the information frame to be accepted by the receiver. The throughput, $\eta$, is given by:

$$
\eta=\frac{k}{N_{a v}}
$$

the parameter $N_{a v}$ can be obtained by:

$$
N_{a v}=\sum_{i=0}^{+\infty} N_{i} p_{i},
$$

where $N_{i}$ is the total number of bits transmitted after the $i$ th transmission and $p_{i}$ is the probability of the acceptance of the frame by decoder after the $i$ th transmission and is given by:

$$
p_{i}=\left(1-F_{i}\right) \prod_{j=0}^{i-1} F_{j}
$$

In (4.3), $F_{i}$ is the frame error rate, an alternative term for WER in ARQ literature, of the code used in the $i$ th transmission. Also in deriving (4.3), it is assumed that decoding failures at different transmission stages are independent.

\section{4-2-2 Capacity}

We may use channel capacity to upper bound system throughput. Given that the input to the AWGN channel is discrete, taking on binary values, \pm 1 , then we can use the formula for the capacity of binary-input AWGN channel [39]: 


$$
C^{*}=\frac{1}{2} \sum_{i=0}^{1} \int_{-\infty}^{+\infty} p\left(y \mid x_{i}\right) \log _{2}\left(\frac{p\left(y \mid x_{i}\right)}{\frac{1}{2} \sum_{k=0}^{1} p\left(y \mid x_{k}\right)}\right) d y,
$$

where $x_{i}=1-2 i(i=0,1), p\left(y \mid x_{i}\right)$ denotes the conditional probability density function of the channel output, $y$, conditioned on the channel input, $x_{i}$. Equation (4.4) can be more simplified as following:

$$
\begin{aligned}
C^{*} & =\frac{1}{2} \sum_{i=0}^{1} \int_{-\infty}^{+\infty} p\left(y \mid x_{i}\right)\left\{1+\log _{2}\left(\frac{p\left(y \mid x_{i}\right)}{\sum_{k=0}^{1} p\left(y \mid x_{k}\right)}\right) d y\right. \\
& =1+\frac{1}{2} \sum_{i=0}^{1} \int_{-\infty}^{+\infty} p\left(y \mid x_{i}\right) \log _{2}\left(\frac{p\left(y \mid x_{i}\right)}{\sum_{k=0}^{1} p\left(y \mid x_{k}\right)}\right) d y \\
& =1-\frac{1}{2} \sum_{i=0}^{1} \int_{-\infty}^{+\infty} p\left(y \mid x_{i}\right) \log _{2}\left(\frac{\sum_{k=0}^{1} p\left(y \mid x_{k}\right)}{p\left(y \mid x_{i}\right)}\right) d y .
\end{aligned}
$$

For the AWGN channel, $p\left(y \mid x_{i}\right)$ is given by

$$
p\left(y \mid x_{i}\right)=\frac{1}{\sqrt{2 \pi \sigma^{2}}} e^{-\frac{1}{2 \sigma^{2}}\left(y-x_{i}\right)^{2}},
$$

where $\sigma^{2}=N_{o} / 2 E_{s}$. Substituting (4.6) into (4.5) yields:

$$
\begin{aligned}
C^{*} & =1-\frac{1}{2} \sum_{i=0}^{1} \int_{-\infty}^{+\infty} p\left(y \mid x_{i}\right) \log _{2}\left(\frac{\sum_{k=0}^{1} \frac{1}{\sqrt{2 \pi \sigma^{2}}} e^{-\frac{1}{2 \sigma^{2}}\left(y-x_{k}\right)^{2}}}{\frac{1}{\sqrt{2 \pi \sigma^{2}}} e^{-\frac{1}{2 \sigma^{2}}\left(y-x_{i}\right)^{2}}}\right) d y \\
& =1-\frac{1}{2} \sum_{i=0}^{1} \int_{-\infty}^{+\infty} p\left(y \mid x_{i}\right) \log _{2}\left[\sum_{k=0}^{1} e^{-\frac{1}{2 \sigma^{2}}\left[\left(y-x_{k}\right)^{2}-\left(y-x_{i}\right)^{2}\right]}\right] d y .
\end{aligned}
$$


Note that $e^{\frac{1}{2 \sigma^{2}}\left[\left(y-x_{k}\right)^{2}-\left(y-x_{i}\right)^{2}\right]}=1$ if $x_{i}=x_{k}$, and when $x_{i} \neq x_{k}$, we have $x_{i}=-x_{k}$. The last equation can then be simplified as follows:

$$
\begin{aligned}
C^{*} & =1-\frac{1}{2} \sum_{i=0}^{1} \int_{-\infty}^{+\infty} p\left(y \mid x_{i}\right) \log _{2}\left[1+e^{\frac{1}{2 \sigma^{2}}\left[\left(y+x_{i}\right)^{2}-\left(y-x_{i}\right)^{2}\right]}\right] d y \\
& =1-\frac{1}{2} \sum_{i=0}^{1} \int_{-\infty}^{+\infty} p\left(y \mid x_{i}\right) \log _{2}\left[1+e^{\frac{-2 y x_{i}}{\sigma^{2}}}\right] d y \\
& =1-\int_{-\infty}^{+\infty} p\left(y \mid x_{0}\right) \log _{2}\left[1+e^{\frac{-2 y x_{0}}{\sigma^{2}}}\right] d y,
\end{aligned}
$$

where in the last step, we have used the fact that the two integrals for $i=0$ and $i=1$ are equal. Next, we make the substitution $w=\left(y-x_{0}\right) / \sigma$, such that (4.7) simplifies to:

$$
C^{*}=1-\int_{-\infty}^{+\infty} p(w) \log _{2}\left[1+e^{\frac{-2\left(1+o w x_{0}\right)}{\sigma^{2}}}\right] d w
$$

In (4.8) $p(w)$ is the probability density function of a zero-mean, unit-variance Gaussian random variable. Evaluating (4.8) with $x_{0}=1$ and $\sigma^{2}=N_{0} / 2 E_{s}$, we obtain the final result

$$
C^{*}=1-\int_{-\infty}^{+\infty} p(w) \log _{2}\left[1+e^{-\frac{4 E_{s}}{N_{0}}} e^{-2 w \sqrt{\frac{2 E_{s}}{N_{0}}}}\right] d w
$$

There is no closed form expression for the capacity in (4.9), and, thus, it must be evaluated numerically. The curve from equation (4.9) is depicted in Fig. 4.1 as a function of symbol signal to noise ratio. From Shannon's coding theorem, we know that reliable communication can be achieved with arbitrary low probability of error as long as the 
code rate is less than capacity. Thus for the type-II hybrid-ARQ protocols, the achievable throughput must be below the capacity curve $C^{*}$.

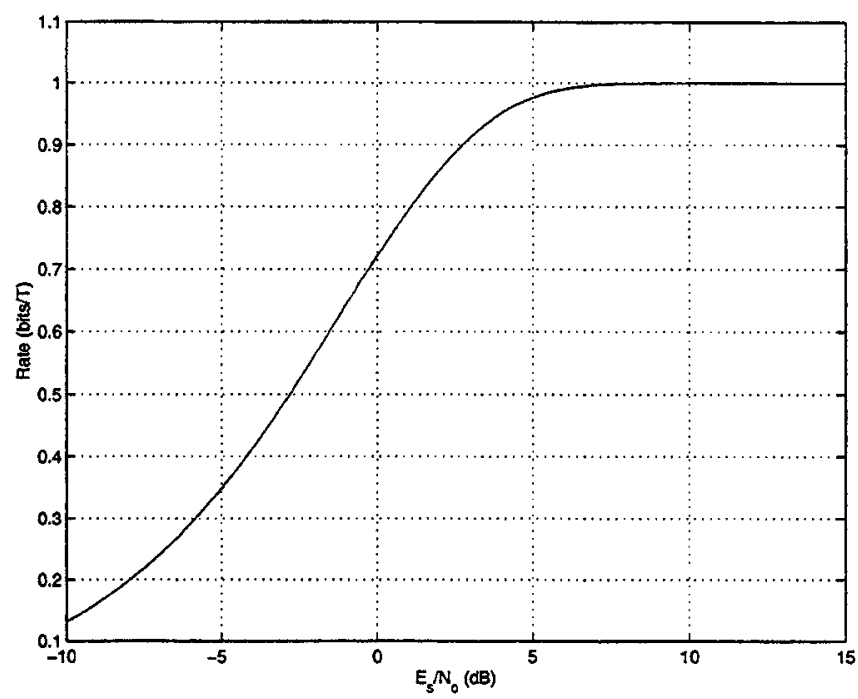

Fig. 4.1. Capacity of a binary-input, AWGN channel

\section{4-3 Type-II Hybrid-ARQ Protocols Using RCPT Codes}

A turbo encoder is a parallel concatenation of multiple recursive convolutional codes, each separated by a pseudo-random interleaver [3]. Turbo decoding is performed by softinput, soft-output (SISO) decoding blocks which use the forward-backward algorithm, to implement a posteriori probability (APP) decoding [3]. Since there are multiple concatenated encoders, the decoder employs multiple APP decoding blocks in an iterative structure within which the decoders pass information. A variety of code rates can be obtained from turbo codes by changing the number of constituent encoders in the parallel concatenation and puncturing the resulting outputs. The use of RCPT codes was independently proposed in [40], [41], and [42]. In all of these works, incremental parity frames are generated by puncturing a mother code. The type-II hybrid-ARQ protocol based on RCPT codes works as following: First an $(n, k)$ CRC code is used to encode the block of $k$ in- 
formation bits and then these $n$ bits go into a low rate RCPT encoder. The resulting systematic and parity bits are stored in the transmitter. Then depending on the plan of transmission, a percentage of the parity bits, say $5 \%$, are sent along with the systematic bits. At the receiver, the decoder substitutes erasure in the place of punctured parity bits and goes through the decoding procedure by using a standard iterative turbo decoder. After each iteration, the decoder computes a CRC check and uses it as an early termination criterion. If there are still errors in the decoded bits, after a prescribed maximum number of iterations, a NAK is sent back to the transmitter. Then the transmitter sends a new subset of parity bits. The receiver attempts the decoding by using the new parity bits along with the previously received bits. This procedure continues until either the CRC check is satisfied or all the parity bits are sent. In the latter case the protocol is reset and again starts by going through the same transmission procedure for the same set of information bits. In this case the receiver may either reset the receive buffer or, retain all previously received symbols and combine them with the newly received symbols [43]. In general, packet combining is performed by averaging the soft-decision values obtained from multiple copies. In [43], it has been shown that this is equivalent to maximum-likelihood diversity combining.

The best throughput performance for type-II hybrid-ARQ protocol based on RCPT codes at short lengths has been reported in [45], where the puncturing pattern is different from previous similar work in [46] and also the parity increment sizes are more uniform than those of the work in [46]. The parity increment size in [45] is $1 / 10$ of the total amount of parity bits, with the exception of the first two transmitted parity blocks which are $1 / 20$ of the total amount of parity bits. As a result, the family of RCPT code contains 
the following 11 code-rates: $0.91,0.83,0.71,0.62,0.56,0.50,0.45,0.42,0.38,0.36$, and 0.33. Also the memory length of the constituent codes is 4 . Fig. 4.2 shows the throughput curve obtained in [45] for data block length $k=1000$ and $\mathrm{CRC}$ length equal to 16 over an AWGN channel, along side the channel capacity. As it can be seen, in some points the throughput is within $1 \mathrm{~dB}$ of the channel capacity.

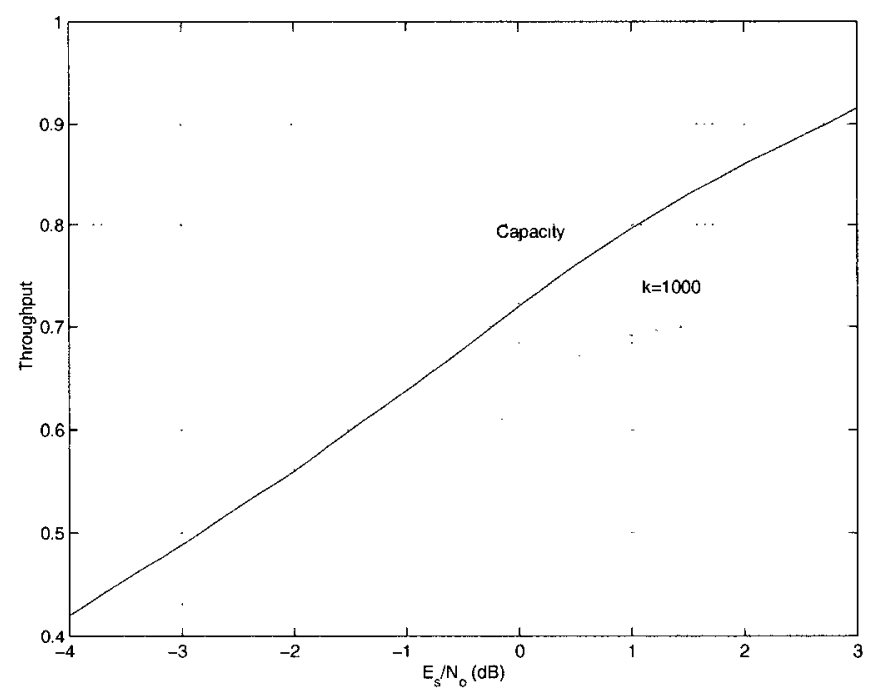

Fig. 4.2. Throughput of the type-II hybrid-ARQ system using RCPT codes, from [45].

\section{4-4 Type-II Hybrid ARQ Protocols Based on RC-LDPC Codes}

Let us look more carefully at the following equation that can be obtained for the throughput of a type-II hybrid ARQ protocol based on RC codes, by combining (4.1), (4.2), and (4.3):

$$
\eta=\frac{k}{\sum_{i=0}^{+\infty} N_{i}\left(1-F_{i}\right) \prod_{j=0}^{i-1} F_{j}}
$$

It can be seen from (4.10) that, assuming a fixed number of nested codes with fixed block lengths, the WER performance of RC codes is the determining factor of the throughput. Thus for obtaining the best throughput efficiency, we have to find a family 
of RC codes that have the best WER performance. The problem of finding a good family of RC-LDPC codes at very long block lengths has been addressed in [47], and [48], where the density evolution techniques have been employed to introduce a systematic way for RC puncturing of a single LDPC code such that the code remains good across a wide range of punctured rates. However, the violation of the underlying cycle-free assumptions of density-evolution techniques at short block lengths, prevents us from using these techniques for finding a good family of RC codes at short block lengths. In this thesis we have been searching for a family of RC-LDPC codes that yield a good WER performance at short block lengths and as we will show in this section, we have come up with the conclusion that, employment of linear PEG-RC-LDPC codes in type-II hybridARQ protocols gives a very good throughput performance, while having the benefit of linear time encoding as well.

We start this section by describing two methods that can be used for obtaining a family of RC-LDPC codes and then explain how a proper combination of these methods can be used to obtain a family of RC-LDPC codes with good performance. Then we apply this approach to a code with a simple irregular profile to show that irregular RC-LDPC codes have the potential to outperform both RCPT codes and regular RC-LDPC codes. The performance of the latter has been reported in [49]. Finally, we construct a family of irregular PEG-RC-LDPC codes for data block length $k=1024$, which results in a throughput considerably better than all the previously reported results at the same block length and at the same time has a low encoding complexity. The same construction principles can be applied to other block lengths or other choices of incremental parity-block sizes. Before going through the next subsection we remind that the strong error detection capa- 
bility of LDPC codes removes the need for using a CRC code in the type-II hybrid-ARQ protocols.

\section{4-4-1 Constructing RC-LDPC Codes}

Generally, there are several useful techniques that can be used to obtain new codes from existing ones. For constructing RC-LDPC codes, two of these techniques, puncturing and extending, are very helpful and will be described in this sub-section.

\section{4-4-1-1 Puncturing}

Puncturing can be used to generate a nested family of higher rate codes from an LDPC code, in the same way that was explained for turbo codes; each member of the family has some part of the parity bits of the mother code as its parity bits and the party bits of each code are included in the parity bits of all lower rate codes. The decoding of each code can be done in the decoder of the mother code by inserting erasures for the punctured parity bits.

In order to evaluate the performance of the codes obtained by puncturing of LDPC codes, we have punctured two LDPC codes and simulated the performance of obtained codes under BP algorithm. The first code is a rate-16/24 $(3,9)$-regular LDPC code with $k=2048$ and $n=3072$. The second code is an irregular rate-8/14 code with $k=1024$, $n=1792$, in which $k / 2=512$ symbol nodes have degree 2 and all other symbol nodes are of degree 3 and the check node degrees are designed to be as concentrated as possible which results in 512 check nodes with degree 6 and 256 nodes with degree 7 . We have constructed both of these two codes using the random Poisson construction by avoiding the cycles of length 4 . The first code has been randomly punctured to give 4 higher rate codes with rates $16 / 23,16 / 22,16 / 21$, and $16 / 20(128,256,384$, and 512 bits have been 
punctured, respectively). For the second code $128,256,384$, and 512 bits of degree 2 have been punctured to obtain 4 codes with rates $8 / 13,8 / 12,8 / 11$, and $8 / 10$, respectively. Other than the above constraint on the degree of punctured bits, the puncturing is performed in random. The performance of these codes is shown in Fig. 4.3 and Fig. 4.4. Note that all codes are decoded using the decoder of the mother code by replacing the information for the punctured parity bits by erasures. For all simulation results of this chapter, maximum number of iterations is equal to 500 and for each signal to noise ratio enough noisy codewords are simulated to generate at least 200 codeword errors. Also in these figures, the performance of typical LDPC codes with the same parameters has been presented, for the sake of comparison. From now on, when we want to evaluate the performance of a code obtained by puncturing a mother code, we use the word "typical" to refer to a code that has the same degree profile as that of the mother code and has been constructed based on the same construction as that of the mother code; for example in Fig. 4.4, since for the mother code, $k / 2$ of symbol nodes have degree 2 and the rest have degree $3, k / 2$ of symbol nodes of typical codes have degree 2 and the rest have degree 3 . Also similar to the mother code, typical codes have check degrees that are made as concentrated as possible and have been constructed based on the random Poisson construction by avoiding the cycles of length 4 . From these two figures it can be observed that when the amount of puncturing is small, and thus the rate of code after puncturing is close to the rate of the mother code, the WER curves are close to the WER curves of typical LDPC codes with the same rate. However, as the number of punctured bits increases, the loss in the performance increases and for the codes with highest rates, this deviation in performance is considerably high. 


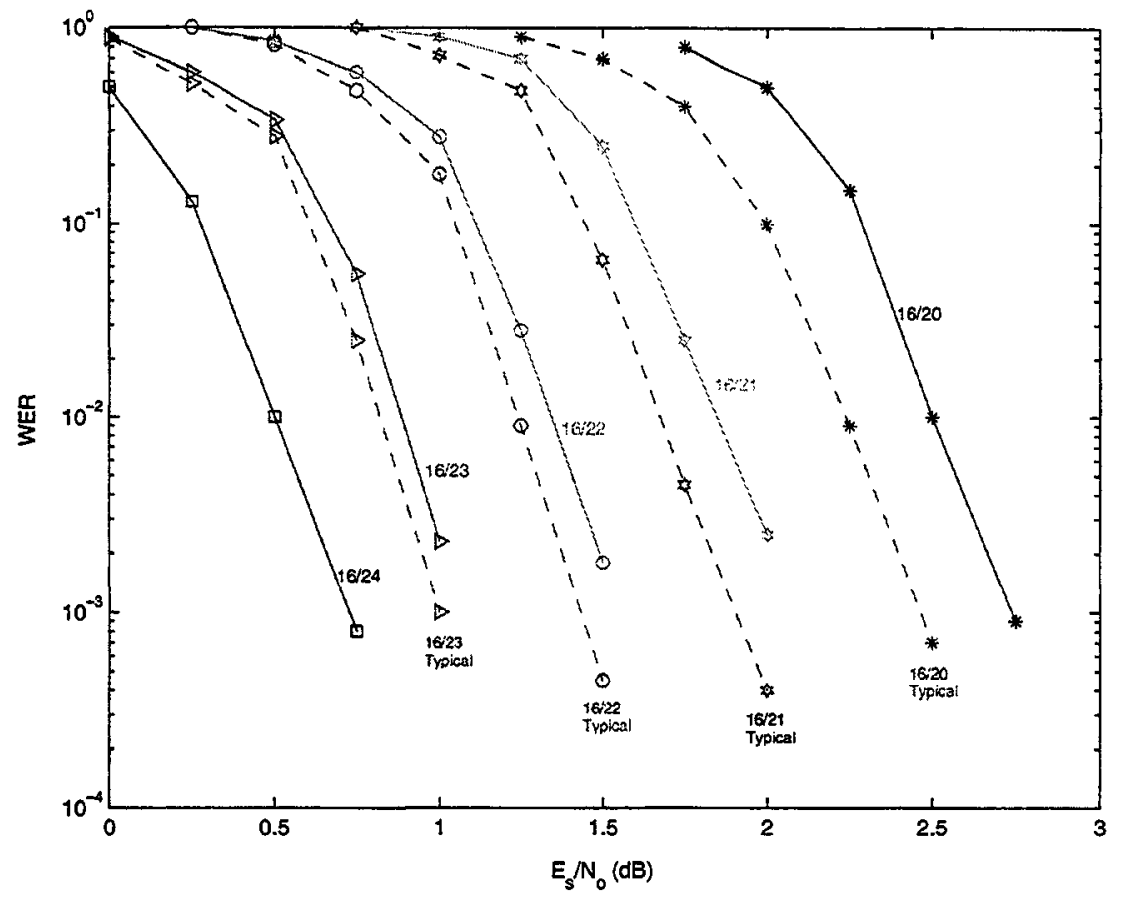

Fig. 4.3. Performance of codes obtained by puncturing a $(3,9)$-regular LDPC code of rate-16/24 with $k=2048$. Solid lines: Performance of RC codes obtained by puncturing. Dashed lines: Performance of typical codes with the same block lengths and rates as punctured codes.

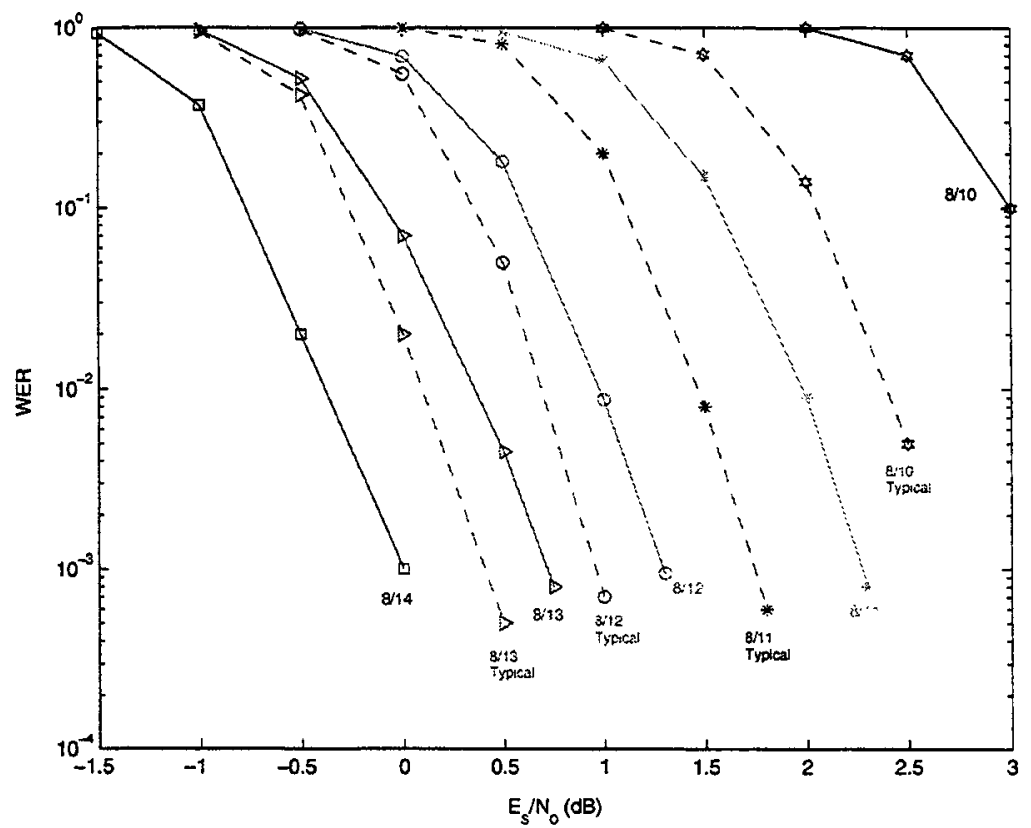

Fig. 4.4. Performance of codes obtained by puncturing an irregular LDPC code of rate- $8 / 14$ with $k=1024$. Solid lines: Performance of RC codes obtained by puncturing. Dashed lines: Performance of typical codes with the same block lengths and rates as punctured codes. 
The reason behind this phenomenon can be explained by noticing the messages that are sent back by a check node $c$ to a symbol node $s$ connected to $c$, after receiving all the incoming messages from the neighbor symbol nodes, during the BP algorithm:

$$
m_{c \rightarrow s}=\log \frac{1+\prod_{k \in N(c) i s} \tanh \left(0.5 m_{k \rightarrow c}\right)}{1-\prod_{k \in N(c) i s} \tanh \left(0.5 m_{k \rightarrow c}\right)}
$$

Consider a check node $c$ in which two or more of incoming messages from symbol nodes are erasures ( 0 in log-likelihood ratio domain); from (4.11) it can be seen that all of the outgoing messages from this check node to its neighboring symbol nodes will be erasure messages. In other words, this check node does not exchange any information with other parts of the graph. Now if the percentage of such parity check nodes is high, then through successive iterations of $\mathrm{BP}$, not much extrinsic information is sent towards erased parity bits and so the algorithm does not converge to the right codeword.

\section{4-4-1-2 Extending}

The opposite process to puncturing a code is extending in which some additional parity bits are added to the codewords. A family of RC codes can be obtained by successively extending an LDPC code. Obviously, when we extend an LDPC code to a lower rate code, some new rows and columns are added to the parity-check matrix of the code; the addition of these new rows and columns have to be performed in such a way that the rate-compatibility is not violated and the performance of the new code is as close as possible to the performance of a typical LDPC code with the same rate. An appropriate structure that fits both of these two purposes is illustrated in Fig. 4.5 [49]. 


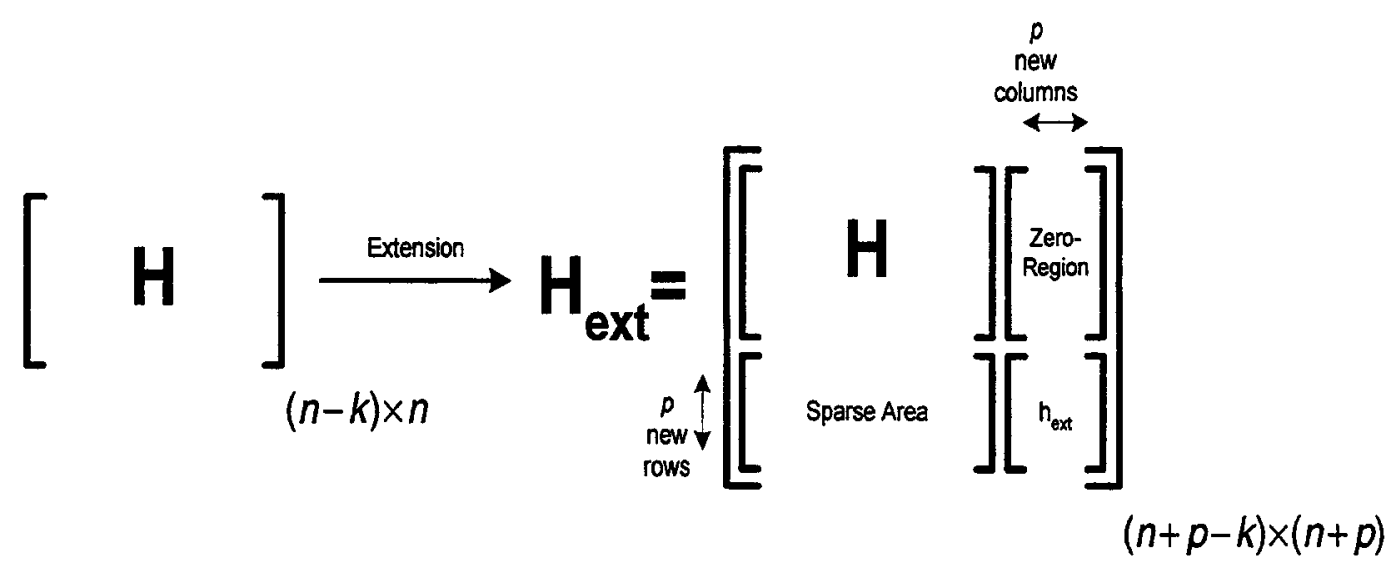

Fig. 4.5. Extension of LDPC codes

In this figure the parity-check matrix, $H$, of a rate- $k / n$ LDPC code is extended to the parity-check matrix, $H_{\text {ext }}$ of a rate $k /(n+p)$ code. By setting the entries of the Zero-Region of $H_{\text {ext }}$ to zero, it's guaranteed that the parity bits of $H$ are contained in the parity bits of $H_{\text {ext. }}$. Because of this restriction on the newly added columns of $H_{\text {ext }}$, we have to satisfy the weight constraints of these columns by the square matrix $h_{\text {ext }}$ in the bottom-right part of $H_{\text {ext }}$.The bottom-left area, which is denoted by Sparse-Area in Fig. 4.5, is used to provide dependency between the code bits of $H$ and the newly added parity-bits. This area has to be sparse in order to not change the symbol-degree distribution of $H$ considerably. The procedure of extending can be applied successively to obtain lower rate codes.

Figs. 4.7 and 4.9 show the BP-WER performance of the extended codes obtained by extending a rate-4/8, $(3,6)$-regular LDPC code with $n=2048, k=1024$ and extending the irregular LDPC code that was used for puncturing in Fig. 4.4, respectively. The rate-4/8 code has been constructed based on the random Poisson construction by avoiding the cycles of length 4 . The parity-check matrix of the rate- $4 / 8$ code has been extended 4 times to give 4 new parity-check matrices that correspond to 4 lower rate codes with rates $4 / 9$, $4 / 10,4 / 11$, and $4 / 12$. In all of these extensions, the extension matrix of Fig. $4.5, h_{\text {ext }}$ is a 


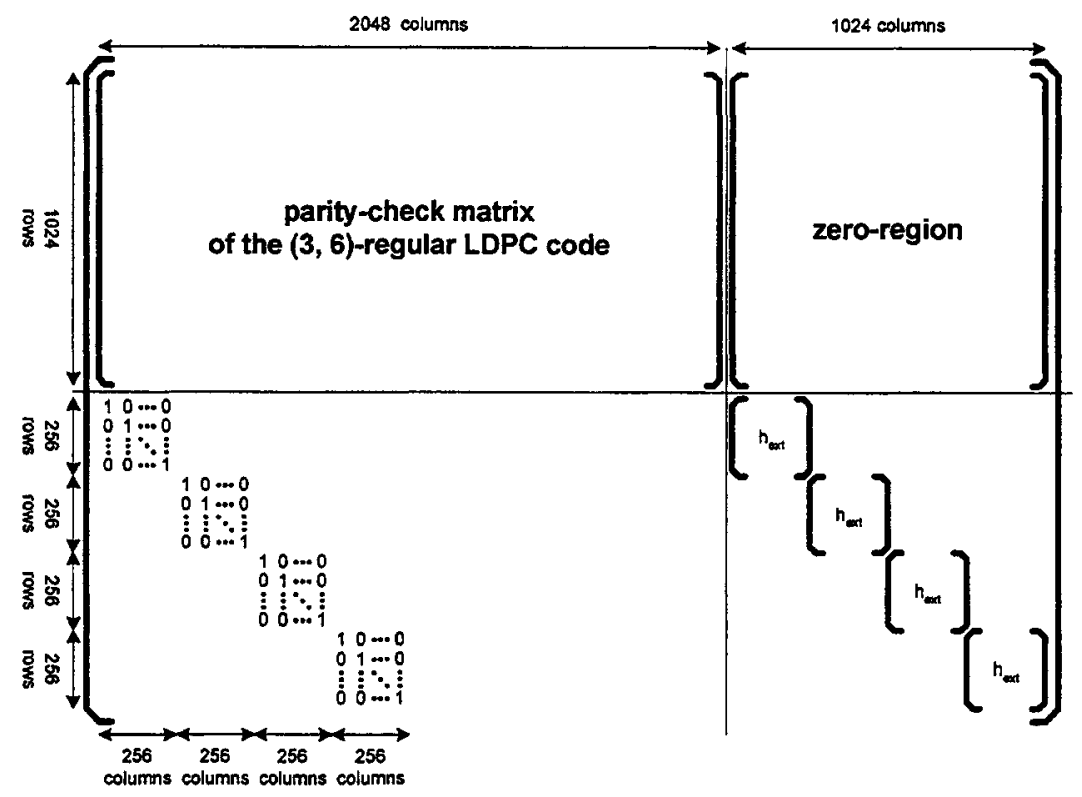

Fig. 4.6. Illustration of the extension of the rate-4/8, $(3,6)$-regular LDPC code with $k=1024$.

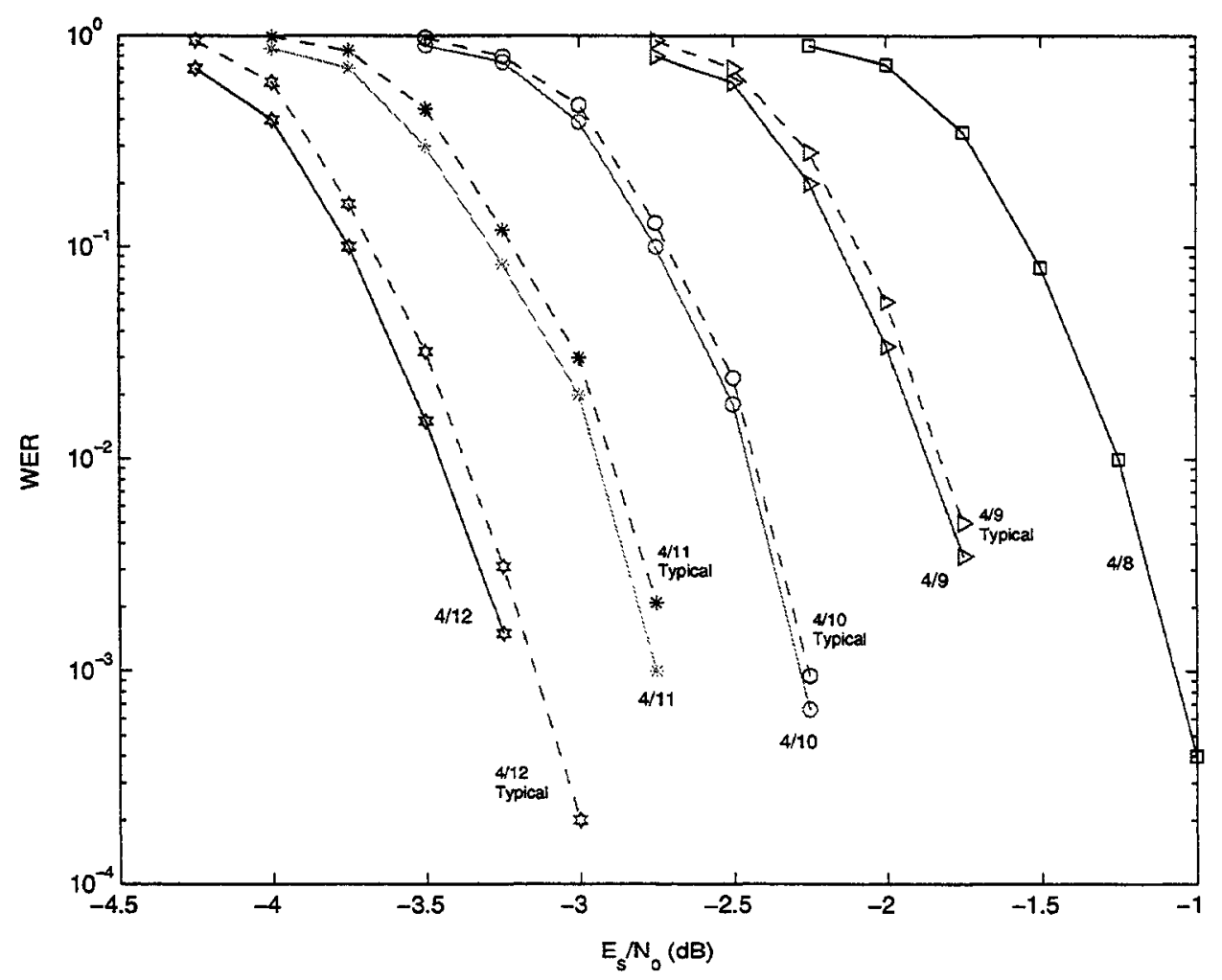

Fig. 4.7. Performance of codes obtained by extending a regular- $(3,6)$ LDPC code of rate- $4 / 8$ with $k=1024$. Solid lines: Performance of RC codes obtained by extending. Dashed lines: Performance of typical codes of the same block lengths and rates as extended codes. 


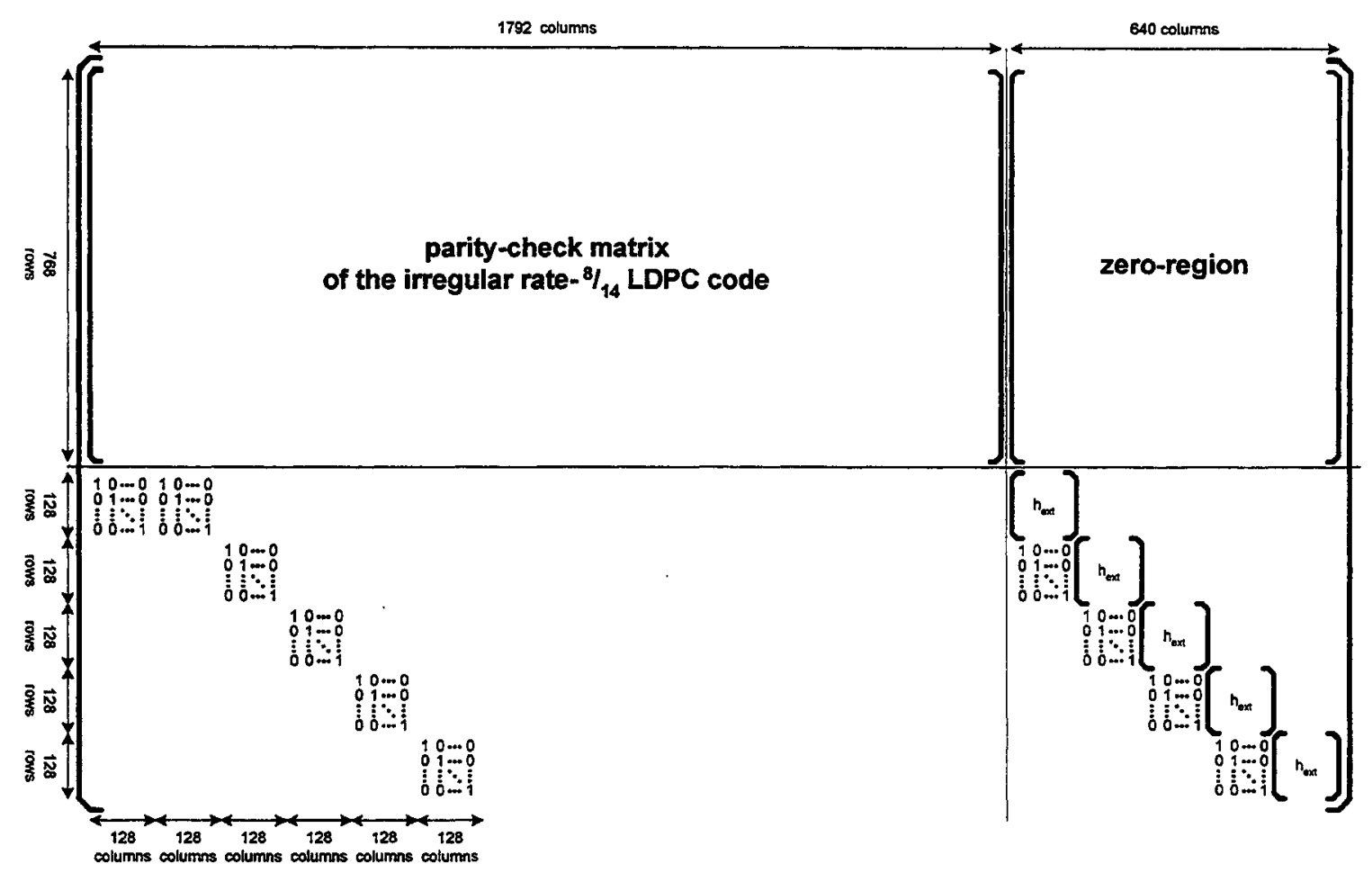

Fig. 4.8. Illustration of the extension of the rate-8/14, irregular LDPC code with $k=1024$.

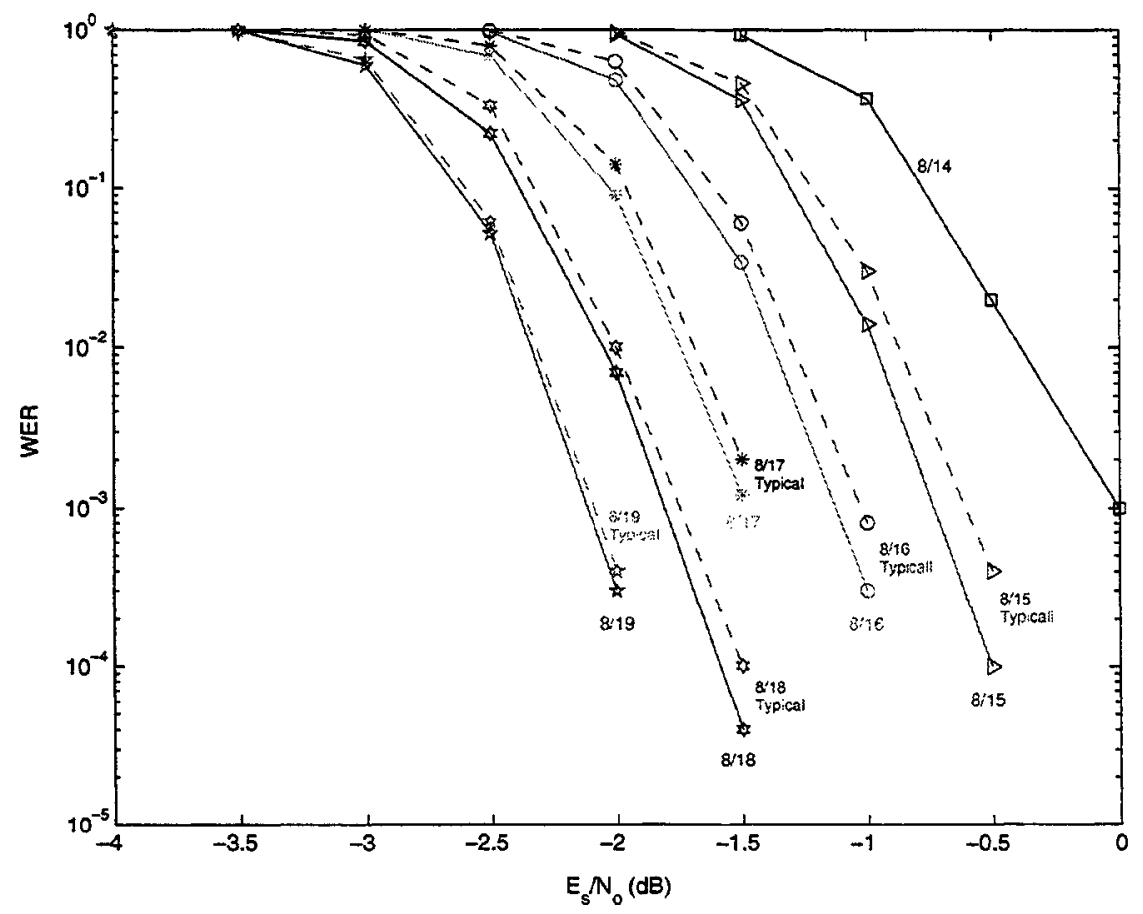

Fig. 4.9. Performance of codes obtained by extending an irregular LDPC code of rate-8/14 with $k=1024$. Solid lines: Performance of RC codes obtained by extending. Dashed lines: Performance of typical codes of the same block lengths and rates as extended codes. 
$256 \times 256$ matrix with column weights and row weights equal to 3 and there is only one "1" in the sparse area of each newly added row of the matrix. Fig 4.6 illustrates the procedure that has been used for extending this matrix. The extension matrix, $h_{\text {ext }}$, has been constructed based on the random Poisson construction by avoiding cycles of length 4 .

For extending the parity-check matrix of the irregular rate- $8 / 14$ code, we have tried to find an extension matrix, $h_{\text {ext }}$, which gives the best performance for the extended codes. For example, since the irregular rate-8/14 code has 512 check nodes with degree 6 and 256 check nodes with degree 7 , we started our heuristic search based on the right foresight that for the first extension, adding rows of weight 7 is a proper approach for the extended rate-8/15 code, because it preserves the uniformity of row weights. However, there are several combinations of the extension matrix, $h_{e x t}$, and the sparse area which result in row weight 7 . We started the search with a regular- $(3,3)$ extension matrix and four 1 's in each row of sparse area, and then modified the extension matrix and the sparse area several times to find the best extension matrix. The best extension matrix that we were able to find for the rate- $8 / 15$ code is a $128 \times 128$ matrix with column and row weights equal to 5 which has been constructed based on the random Poisson construction by avoiding the cycles of length 4 . Also in the sparse area of newly added rows to the paritycheck matrix, two 1's have been placed in each row as shown in Fig. 4.8. This figure illustrates the best configuration that we have been able to find, in terms of WER performance of the extended codes. An important advantage of this configuration is that meanwhile having a systematic form which allows us to easily implement the same configuration at other block lengths and code rates, it doesn't generate many cycles of length 4 in the Tanner graph of the extended codes. In fact, due to this special pattern of 1 's in the 
sparse area, we just have the possibility of producing cycles of length 4 in the first extension of the matrix (assuming that the extension matrix, $h_{\text {ext }}$, does not contain cycles of length 4). For our extension, the rate $8 / 15$ code just has four cycles of length 4 which practically does not have any negative effect on the performance of extended codes. It is also worth mentioning that through our search for finding the best extension configurations, we have considered those combinations of extension matrices and sparse area which violate the uniformity of row weights of extended matrix. However, the results have been worse than the combinations which make the row weights of extended matrix uniform.

Construction of RC-LDPC codes with extending has some advantages over using puncturing for the same purpose. The first advantage is that when we want to use our family of RC codes in the type-II hybrid-ARQ protocol, the extra parity bits of each code are generated only when we need to send them, whereas in puncturing usually all of the parity bits of the mother code are generated at the encoder at the beginning of sending each data packet. The more important advantage is that the decoding of punctured codes has to be done in the decoder of the mother code by inserting erasures at the locations of punctured bits while, effectively, the decoding of each extended code is performed in its own decoder. This results in both better performance and less computational complexity for the codes that are obtained by extending in comparison with the codes that are obtained by puncturing. To make this point more clear, note that if the row weights of $h_{\text {ext }}$, in the bottom-right part of $H_{\text {ext }}$ in Fig. 4.5, are at least 2 and we decode the extended codes using the decoder of the lowest rate code (and inserting erasure in the locations of missing parity bits), all the rows corresponding to missing parity bits become redundant 
due to the effect of erasures on them. But since erasures don't have any effect on the original check nodes of the extended codes, effectively, we will have a standard BP decoder for each extended code (even when we turn off that part of the decoder that corresponds to the missing parity bits).

For comparison purposes, also displayed in Figs. 4.7 and 4.9, for each extended matrix, is the BP-WER performance of a typical LDPC code with similar parameters. In evaluating the performance of the codes obtained by successive extension of an LDPC code $C$, we use the term "typical" to refer to the codes which have the same profile of the code $C$ and have been generated by using the same construction method of $C$. We observe that for these two codes, the WER performance of the extended codes is always slightly better than the performance of a typical LDPC code with the same parameters. The reason is that, for both of these two codes, extended codes have a larger average column weight than that of the typical LDPC codes, which results in a better performance for the code. In fact, for each of the codes obtained by extending, normally the performance can not be better than the performance of a code which has the same symbol-degrees of this code and has been constructed using the Poisson construction, by avoiding the cycles of length 4 and by making check-degrees as concentrated as possible. This is because of the inevitable constraints that are imposed on the Tanner graph of the codes which are obtained by extending. In Fig. 4.10 , we have compared the performance of the rate-8/16 code which was obtained by extending the rate-8/14 code of Fig. 4.9 , with the performance of a rate-8/16 code with $k=1024$, which has the same symbol-degree distribution of the other code and has been constructed using the Poisson construction, by avoiding the cycles of length 4 and by making check-degrees as concentrated as possible. The result, 
as expected, is that the performance of the Poisson-constructed code is always slightly better than the performance of the code obtained by extending.

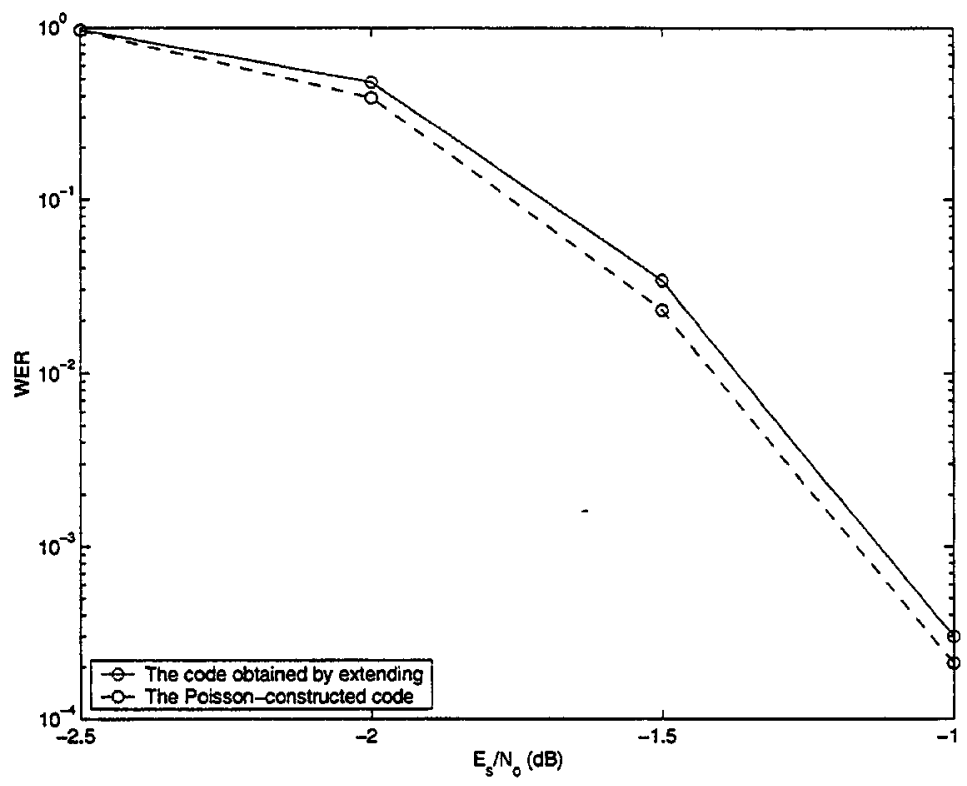

Fig. 4.10. Comparing the performance of the rate- $8 / 16$ code of Fig.4.9, obtained by extending, with a similar rate- $8 / 16$ code, obtained by Poisson construction.

The only problem with extending is that if we start extending from a high rate code, it fails to provide good lower rate LDPC codes. This is because the row weights of the parity-check matrix of a high rate LPDC code are quite large and thus the newly added rows to this matrix do not improve the performance sufficiently, unless they have weights close to the large weights of the old rows. On the other hand, since $h_{\text {ext }}$ is a fairly small square matrix, we cannot increase its row weights without having a large number of small cycles in the Tanner graph of this matrix which considerably degrades the performance of extended code. Similarly; increasing the row weights by inserting a large number of 1 's in the sparse area, produces a large number of cycles of length 4 in the Tanner graph of the code. This inevitable considerable difference in the row weights tends to a poor performance for the extended code. 


\section{4-4-2 Construction of an Efficient Family of RC-LDPC Codes}

As it was described in the last sub-section, both puncturing and extending can be used to generate a family of RC-LDPC codes. However, none of these two methods have the potential to construct a good family of RC-LDPC codes with a wide range of rates; based on our observations, a good performance for puncturing is limited to the range of rates not higher than $30 \%$ of the rate of the mother code and extending can not generate good codes if we start from a high rate code. Thus for constructing an efficient family of RCLDPC codes, with a wide range of rates, we have to take advantage of both approaches; we could start from an LDPC code with a medium rate and then, obtain the higher rate codes by puncturing this code and the lower rate codes by extending it.

Example 4.1. We have used the above-mentioned method to construct a family of RC-LDPC codes with $k=1024$ and with rates varying from $8 / 11$ to $8 / 19$ by extending and puncturing the rate-8/14 irregular LDPC code that was used as an example in the last subsection. The BP-WER performance of the rate- $8 / 13,8 / 12$, and $8 / 11$ codes, which are obtained by puncturing, have been shown in Fig. 4.4 and for the rates $8 / 15,8 / 16,8 / 17,8 / 18$ and $8 / 19$ which are obtained by extending, the BP-WER curves are given in Fig. 4.7.

Using equation (4.10), we can obtain the throughput of the type-II hybrid-ARQ protocol based on this family of RC-LDPC codes. This throughput curve is shown in Fig. 4.11 along with the throughput curve obtained for the RCPT codes in [45]. Also shown is the throughput curve obtained in [49] for a similar family of RC-LDPC codes with $k=1024$ that have been generated by puncturing and extending a $(3,6)$-regular LDPC 


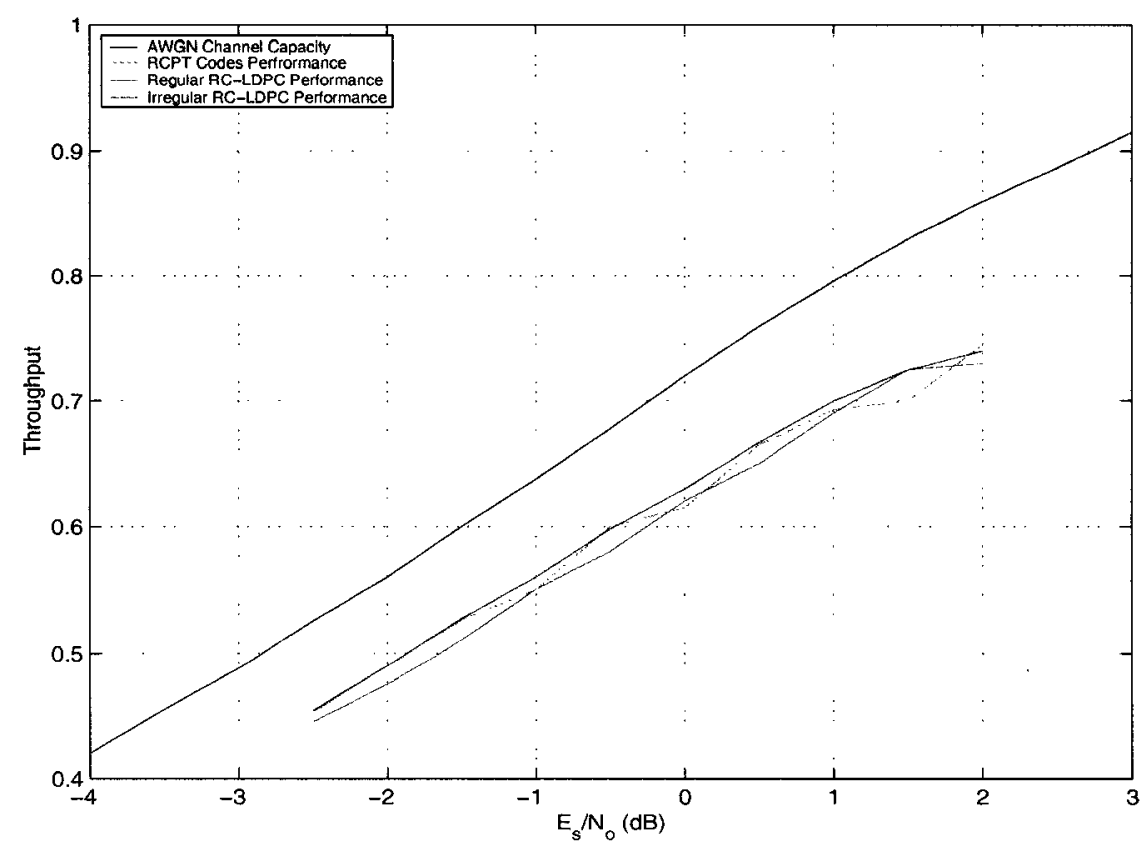

Fig. 4.11. Throughput performance of the type-II hybrid-ARQ protocol based on the family of irregular RC-LDPC codes with $k=1024$, constructed in Example 4.1, and the throughput obtained by regular RC-LDPC codes ([49] ) and RCPT codes ([45] )

code. In comparison with our extending procedure of this irregular rate-8/14 LDPC code, the extending procedure that has been used in [49], is a simple procedure in which just one " 1 " is placed in each row of the sparse area and no effort has been made to provide a good dependency between the code bits of the old parity-check matrix and the newly added parity-bits. Also selection of a $128 \times 128$ matrix with row weights and column weights equal to 3 as the extension matrix, $h_{e x t}$, in [49], is not a good selection because we can get more concentrated row weights for the extended parity-check matrix, if we use a $(5,5)$-regular extension matrix. As a result the performance of the extended codes of the family of RC-LDPC codes in [49] is considerably worse than the performance of corresponding codes in our family, while the two codes that have been used for the first extension $((3,6)$-regular LDPC code in $[49]$ versus the rate-8/14 code in our case) have a very close performance. In Fig. 4.11, we observe that our proposed extension of this 
rather weak irregular LDPC code, tends to a throughput curve which outperforms the throughput curve of the family of RC codes constructed based on the $(3,6)$-regular LDPC code of [49] by up to $0.2 \mathrm{~dB}$. We can expect even more improvement by using RC-LDPC codes that are constructed suitably, based on optimally designed irregular LDPC codes. In the following sub-section we show that irregular PEG-constructed LDPC codes confirm our expectation.

\section{4-4-3 Performance of Type-II hybrid ARQ Protocol Based on PEG- RC-LDPC Codes}

As the simulation results of chapter 2 showed, PEG construction algorithm can be used to construct very good irregular LDPC codes at short block lengths. Also we discussed that, linear-time encoding can be imposed on PEG-constructed LDPC codes without loosing much in terms of performance. This has motivated us to investigate the performance of type-II hybrid-ARQ protocol based on PEG-RC-LDPC codes. In order to be able to compare the performance of this system with existing results for the type-II hybrid-ARQ protocol based on regular LDPC codes, we have selected the same data block length and the same code rates as those of [49] for our family of PEG-RC-LDPC codes. Nonetheless, the method that will be used in the following for constructing the family of PEG-RC-LDPC codes can be easily employed for constructing a family of PEG-RCLDPC codes with other data block lengths, other rates, and other numbers of ratecompatible codes.

The first step in designing our family of RC codes is to construct a central code, which will be punctured and extended to give higher and lower-rate codes of family, re- 
spectively. We have used the following symbol-degree distribution to generate a rate8/13 PEG-RC-LDPC code with $k=1024$ :

$$
\lambda(x)=0.47532 x^{2}+0.279537 x^{3}+0.0348672 x^{4}+0.108891 x^{5}+0.101385 x^{15} .
$$

In selecting the rate of the central code, we have considered the following three facts: 1- Generally the performance of the codes obtained by extending is better than the performance of codes obtained by puncturing. 2- Puncturing can not provide good codes if it is used to construct codes with rates higher than $\% 30$ of the rate of the mother code. 3Extension of high rate LDPC codes does not produce good codes.

As it was mentioned in chapter 2, the degree distribution of (4.12) has been optimized for an irregular-LDPC with rate-1/2 and is not guaranteed to be optimum for a code with rate 8/13. However, the Monte-Carlo approach described in [16] for optimizing the symbol degree distribution of a code with a specific rate is too expensive computationally to be used for obtaining the optimal degree distributions at this rate. Thus we have used the degree distribution of (4.12). Based on this degree distribution and due to the imposed linear-time-encoding restriction on the code, we will have one symbol node with degree 1 and the remaining symbol nodes will be of degree $2,3,4,5$, and 15 with $790,465,58$, 181 , and 169 symbols having each degree, respectively. Also there are 392 and 248 check nodes with degrees 10 and 11 , respectively. The parity-check matrix of code has been generated using the linear PEG construction of subsection 2-1-3, starting with the parity bits in the right side of it and with column weights increasing from right to left. This code has been punctured to generate three higher rate codes with rates $8 / 12,8 / 11$, and $8 / 10$. Sets of 128,256 , and 384 symbol nodes of degree 2 have been randomly punctured to generate three higher rate codes with rates $8 / 12,8 / 11$, and $8 / 10$, respectively. For punc- 
turing we have randomly punctured symbol nodes of degree two in order to have the least possible number of erasures on the edges of the Tanner graph in the decoder.

For extending, we have generated a $128 \times 128$ extension matrix, $h_{\text {ext }}$, with the same irregular symbol-degree distribution of equation (4.12) by using linear PEG-construction algorithm. This selection of the extension matrix, as we will see in the following, tends to provide a good performance for the extended codes while making the linear-time encoding possible. Again here we have one symbol node of degree 1 and there are $60,36,4$, 14 , and 13 symbol nodes with degrees $2,3,4,5$, and 15 respectively. Also there are 13 , $103,10,1$, and 1 check nodes with degrees $5,4,3,2$, and 1 , respectively. The extension matrix has been generated using the deterministic PEG algorithm of subsection 2-1-3, starting from right and with column weights increasing from right to left. The procedure of extending is the same as the procedure of extending the rate-8/14 irregular code of last subsection and is illustrated in Fig. 4.12. As it was discussed before, this extending procedure both reduces the probability of generating cycles of length 4 in the Tanner graph of extended codes and also provides sufficient connection between the newly added parts and old parts of the extended matrices and in overall provides a good performance for the extended codes. Also, because this extension procedure follows a systematic pattern, it can be easily used at other block lengths and code rates.

Note that, since both the parity-check matrix of the rate- $8 / 13$ code, $H$, and the extension matrix, $h_{\text {ext }}$, have been constructed based on the linear-PEG construction, all of the codes will have a linear-time encoding complexity. In fact here, the left part of $H$ corresponds to systematic bits and the parity bits can be successively encoded from left to right by using the check equations from top to bottom, respectively. 
Fig. 4.13 and Fig. 4.14 demonstrate the BP-WER performance of the punctured and extended codes, respectively. Also, for each of the punctured and extended codes, the performance of the corresponding typical code has been depicted. Here, in contrary to what we saw for the extended codes of Figs. 4.7 and 4.9, the performance of extended codes is worse than the performance of the corresponding typical codes. This is because the typical codes that are used for comparison both have a good symbol-degree distribution and have been constructed based on linear-PEG construction and thus their performance is very good. But the extended codes neither have exactly these good symbol-degree distributions and nor have been fully constructed based on linear-PEG construction. As a result the performance of extended codes can not be as good as the performance of typical codes.

The other way of evaluating the performance of each extended code is to compare its performance with a PEG-RC-LDPC code which has the same symbol-degree distribution, the same rate, and the same length as this code. As it was discussed before, depending on the amount of constraints that is put on the Tanner graph of extended codes during their construction, we expect the performance of each extended code to be worse than the performance of its corresponding PEG-RC-LDPC code. This comparison has been done in Figure 4.15 , for the rate-8/14 and rate-8/15 extended codes of Fig. 4.14 . Also the performance of the corresponding typical codes has been shown in this figure. For both code rates, we see that the performance of typical codes is better than the other two codes, which is in accordance with our claim that the typical codes of Fig. 4.14 have very good performances. 
Figure 4.16 shows the throughput curve of type-II hybrid-ARQ protocol based on this family of PEG-RC-LDPC codes along with the throughput curve obtained for the RCPT codes of [45] and the throughput curve obtained in [49] for the RC-LDPC codes with $k=1024$ which have been generated by puncturing and extending a $(3,6)$-regular LDPC code. As we see the throughput curve of our family of RC-LDPC codes outperforms both the throughput curve of RCPT codes and the throughput curve of regular RC-LDPC codes. In fact, this system outperforms the type-II hybrid-ARQ systems of [49] by up to $0.5(\mathrm{~dB})$ and meanwhile has a linear-time encoding complexity.

In order to evaluate the quality of the throughput curve that we have obtained here, we compare it with an upper bound curve which can be obtained for the type-II hybridARQ protocol based on PEG-RC-LDPC codes as following: suppose that we manage to design a family of PEG-RC-LDPC codes with the same rates and block lengths that we considered here, such that the performance of each individual code is as good as the performance of the corresponding typical code. Then the throughput of this system can be considered as an upper bound for the throughput that we obtained in this subsection. In fact, if we recall that the irregular LDPC codes constructed by linear-PEG construction give the best-known WER performances at short block lengths, then this could be considered as an approximate upper bound for the throughput curve that we can obtain by all known constructions of LDPC codes at this block length. This upper bound has been depicted in Fig. 4.17 along with the throughout curve that we obtained for our family of PEG-RC-LDPC codes. As we see from this figure our throughput curve is not far from this upper bound especially at low to moderate values of signal to noise ratio, where the difference is negligible. 


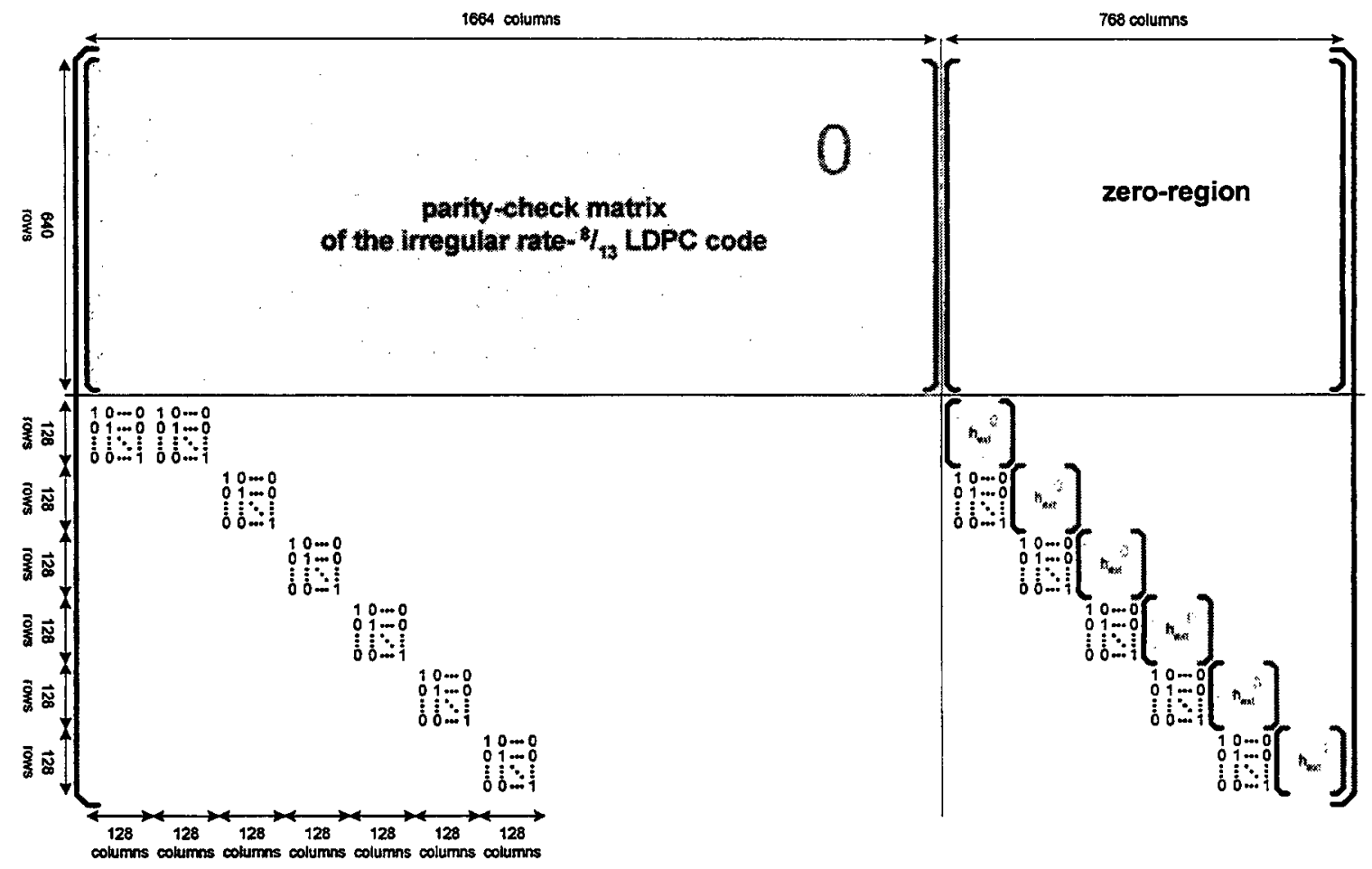

Fig. 4.12. Illustration of the extension of the rate-8/13, PEG-RC-LDPC code with $k=1024$, and the symboldegree distribution of equation (4.12)

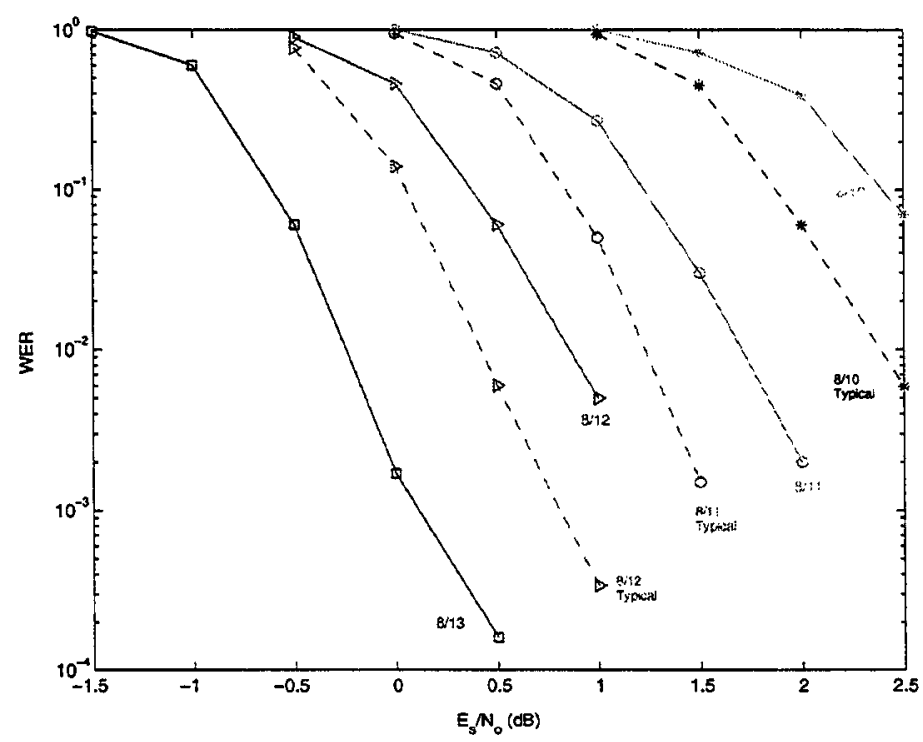

Fig. 4.13. Performance of codes obtained by puncturing a PEG-RC-LDPC code of rate-8/13 with $k=1024$ and with symbol node degree distribution given by equation (4.12). Solid lines: Performance of RC codes obtained by puncturing. Dashed lines: Performance of typical codes with the same block lengths and rates as the corresponding punctured codes. 


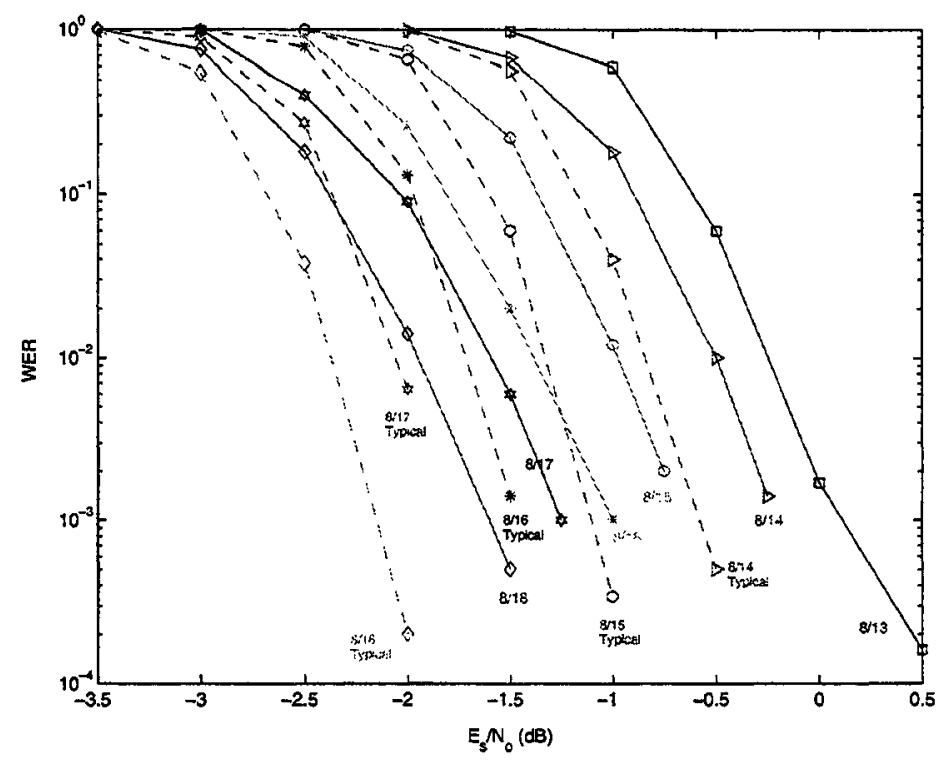

Fig. 4.14. Performance of codes obtained by extending a PEG-RC-LDPC code of rate- $8 / 13$ with $k=1024$ and with symbol node degree distribution given by equation (4.12). Solid lines: Performance of RC codes obtained by extending. Dashed lines: Performance of typical codes with the same block lengths and rates as the corresponding extended codes.

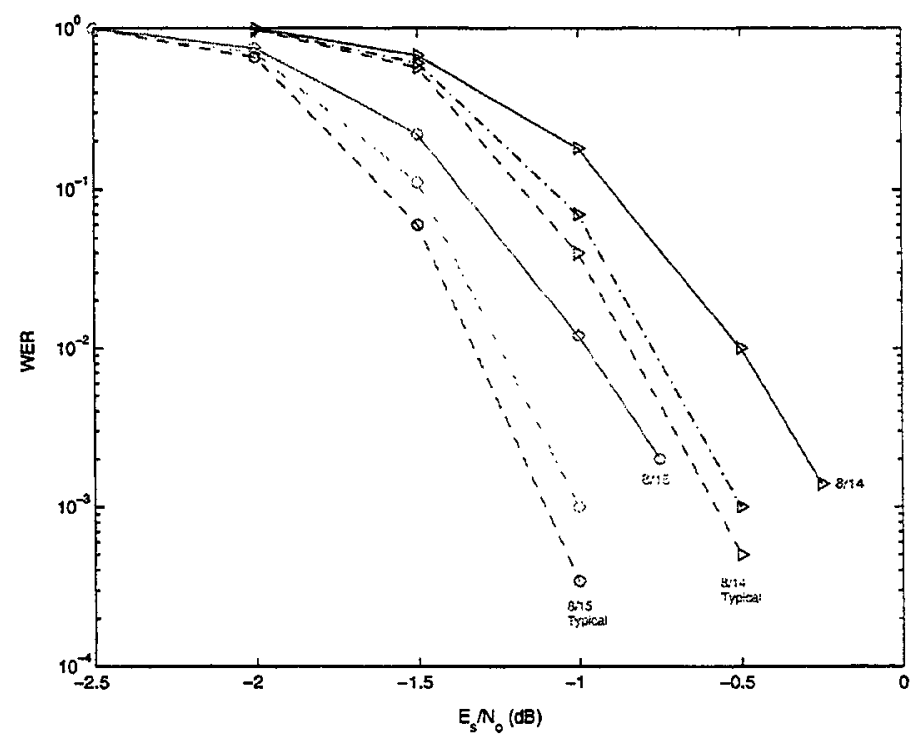

Fig. 4.15. Evaluating the performance of the rate $8 / 14$ and the rate- $8 / 15$ codes of Fig. 4.14 , obtained by extending. Solid lines: Performance of RC codes obtained by extending. Dot-Dashed lines: Performance of the codes with the same block lengths, rates and degree distributions as the corresponding extended codes. Dashed lines: Performance of typical codes with the same block lengths and rates as the corresponding extended codes. 


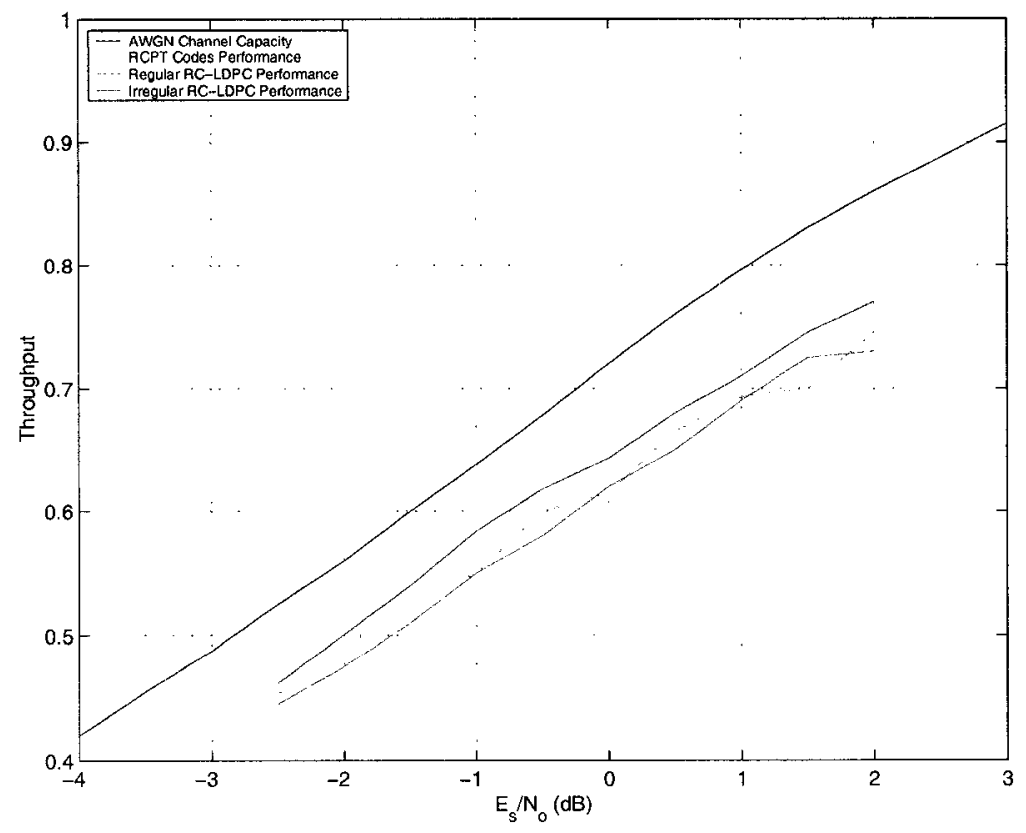

Fig. 4.16. Throughput performance of type-II hybrid-ARQ protocol based on the family of PEG-RCLDPC codes with $k=1024$ and the throughput obtained by similar regular RC-LDPC codes ([49] ) and RCPT codes ([45])

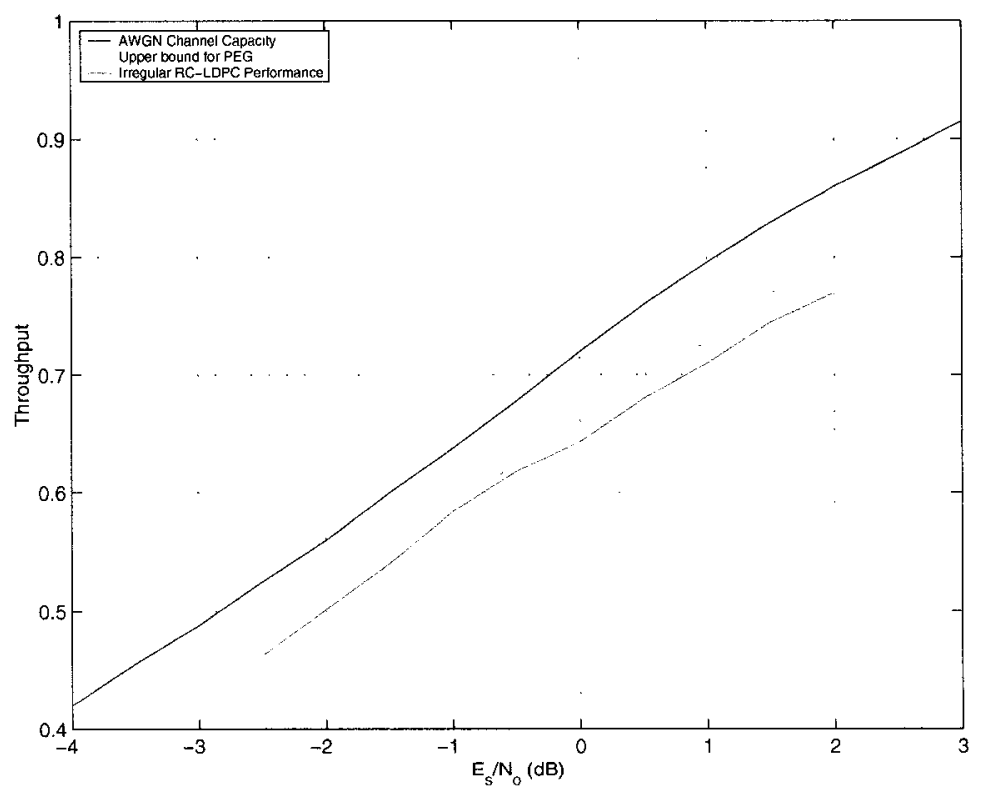

Fig. 4.17. Throughput performance of type-II hybrid-ARQ protocol based on the family of PEG-RCLDPC codes with $k=1024$ in comparison with the upper bound for throughput of PEG-RC-LDPC codes at this data block length. 


\section{4-5 Conclusions}

In this thesis we have studied type-II hybrid-ARQ protocol based on RC-LDPC codes at short block lengths. Different constructions of LDPC codes and the encoding and decoding of LDPC codes were discussed and especially the capability of PEG construction for constructing linear-time encodable LDPC codes with very good performance was emphasized. We then investigated puncturing and extending as two methods that can be used for constructing a family of RC-LDPC codes and discussed the potential advantages and disadvantages of each method. In particular, we devised methods of extending an LDPC code that preserve the structure of LDPC codes during extending and provide extended codes with good performance. In this regard, selecting a suitable structure for the extended parity-check matrix and establishing good connections between the old and new parts of the extended matrix appear to be of critical importance.

A combination of puncturing and extending was employed to generate an efficient family of RC codes based on the linear-time encodable irregular PEG-constructed LDPC codes. This constructed family not only is linear-time encodable but also has a very good throughput. In a data block length of 1024 , this structure outperforms the existing schemes by up to $0.5 \mathrm{~dB}$ over the AWGN channel.

\section{4-6 Ideas for Future Research Work and Outlook}

Packet-oriented data transmission is gaining more and more importance in wireless communication systems. Typically, data transmission on wireless communication channels is not strictly delay-sensitive but requires a virtually error-free link. These two characteristics together with availability of a feedback channel have put up type-II Hybrid- 
ARQ schemes as strong candidates for the channel coding protocol in the next generations of wireless communications [50]. Towards this goal, devising efficient RC codes with low encoding and decoding complexity is the most important step that has to be taken. In this thesis we focused on the throughput performance and the encoding complexity of the family of RC-LDPC codes and for decoding we used the BP algorithm which is considered to be the best iterative decoding algorithm in terms of performance but not in terms of complexity. On the other hand, the quantized versions of Min-Sum algorithm and its minor variations have recently been shown to give very good performance with much lower complexity than BP algorithm [51]. As such, an interesting idea for future research work is to investigate the performance of our family of RC-LDPC codes under these algorithms.

Also, devising LDPC codes that do not lose considerable performance under puncturing is another interesting topic. 


\section{References}

[1] R. G. Gallager, Low-Density Parity-Check Codes. Cambridge, MA: MIT Press, 1963.

[2] R. M. Tanner, "A recursive approach to low complexity codes," IEEE Trans. Inform. Theory, vol. IT-27, pp. 533-547, Sept. 1981.

[3] C. Berrou, A. Glavieux, and P. Thitimajshima, "Near Shannon limit error-correcting coding and decoding: Turbo-codes (I)," in Conf. Rec. 1993 Int. Conf. Comm., Geneva, Switzerland, May 1993, pp. 1064-1070.

[4] N. Wiberg, "Codes and decoding on general graphs," Ph. D. dissertation, Dept. Elec. Eng., Univ. Linkoping, Sweden, Apr. 1996.

[5] F. Kschischang, B. Frey, and A. Loeliger, "Factor graphs and the sum-product algorithm," IEEE Trans. Inform. Theory, vol. 47, no.2 pp. 498-519, Feb. 2001.

[6] R. McEliece, D. MacKay, and J. Cheng, "Turbo decoding as an instance of Pearls 'Belief Propagation' algorithm," IEEE J. Select. Areas Comm., vol. 16, pp. 140-152, Feb. 1998.

[7] S.Y Chung, G.D. Forney, Jr., and T.J. Richardson, R. Urbanke, "On the design of lowdensity parity-check codes within $0.0045 \mathrm{~dB}$ of the Shannon limit," IEEE Comm. Lett. , vol. 5, no. 2, pp. 58-60, Feb 2001.

[8] T. J. Richardson and R. Urbanke, "The capacity of low-density parity-check codes under message-passing decoding," IEEE Trans. Inform. Theory, vol. 47, pp. 599-618, Feb. 2001. [9] Y. Mao and A. H. Banihashemi, "A heuristic search for good low-density parity-check codes at short block lengths," Proc of IEEE Int. Conf. on Comm. (ICC), vol. 1, pp. 41-44, 2001. 
[10] Y. Kou, S. Lin, and M. P. C. Fossorier, "Low-density parity-check codes based on finite geometries: a rediscovery and new results," IEEE Trans. Inform. Theory, vol. 47, pp. 27112736, Nov. 2001.

[11] G. A. Margulis, "Explicit group-theoretical constructions of combinatorial schemes and their application to the design of expanders and concentrators," Problems of Information Transmission (USA), vol. 24, no. 1, pp. 39-46, Jan.-March 1988; translated from Problemy Peredachi Informatsii (USSR), vol. 24, no. 1, pp. 51-60, Jan.-March 1988.

[12] A. Lubotzky, R. Phillips, and P. Sarnak, "Ramanujan graphs," Combinatorica, vol. 8, no. 3, pp. $261-277,1988$.

[13] D. J. C. MacKay, S. T. Wilson, and M. C. Davey, "Comparison of construction of irregular Gallager codes," IEEE Trans.Comm., vol. 47, pp. 1449-1454, Oct. 1999.

[14] X.-Y. Hu, E. Eleftheriou, and D-M. Arnold, "Progressive edge-growth Tanner graphs," Proc. of IEEE Global Telecomm. Conf. (GLOBECOM), San Antonio, Texas, USA, Nov. 2001.

[15] X.-Y. Hu, E. Eleftheriou, and D-M. Arnold, "Irregular progressive edge-growth Tanner graphs," Proc. of IEEE Intl. Symp. Inform. Theory (ISIT), Lausanne, Switzerland, July 2002.

[16] X.-Y. Hu, E. Eleftheriou, and D-M. Arnold, "Regular and irregular progressive edgegrowth Tanner graphs," Submitted to IEEE Trans. Inform. Theory.

[17] L. Bazzi, T. Richardson and R. Urbanke, "Exact thresholds and optimal codes for the binary symmetric channel and Gallager's decoding algorithm A," IEEE Trans. Inform. Theory, to appear. 
[18] M. A. Shokrollahi, "New sequence of linear time erasure codes approaching the channel capacity," Proc. 13th Int. Sym. on Applied Algebra, Algebraic Algorithm and Errorcorrecting Codes, 1999.

[19] M. Luby, M. Mitzenmacher, A. Shokrollahi, D. Spielman, and V. Stemann, "Practical loss-resilient codes," Proc. 29th ACM Symp. Theory of Computing, El Paso, TX, May 1997, pp. 150-159.

[20] D. Spielman, "Linear-time encodable and decodable error-correcting codes," IEEE Trans. Inform. Theory, vol. 42, pp. 1723-1731, Nov. 1996.

[21] L. Ping, W. K. Leung, and N. Phamdo, "Low density parity check codes with semirandom parity check matrix," IEE Elect. Lett., vol. 35, pp. 38-39, Jan. 1999.

[22] H. Jin, A. Khandekar, and R. J. McEliece, "Irregular repeat-accumulate codes," Proc. of 2nd Int. Symp. on Turbo Codes \& Related Topics, Brest, France, Sept. 2000, pp. 1-8.

[23] T. Richardson and R. Urbanke, "Efficient encoding of low-density parity-check codes," IEEE Trans. Inform. Theory, vol. 47, pp. 638-656, Feb. 2001.

[24] D. J. C. MacKay, "Good error-correcting codes based on very sparse matrices," IEEE Trans. Inform. Theory, vol. 45, pp. 399-431, March 1999.

[25] T. M. Cover and J. A. Thomas, Elements of Information Theory. New York: John Wiley and Sons, 1991.

[26] T. Richardson, A. Shokrollahi, and R. Urbanke, "Design of provably good low-density parity-check codes," IEEE Trans. Inform. Theory, vol. 47, pp. 619-637, Feb. 2001.

[27] D. J. C. MacKay, "Online database of low-density parity-check codes," available at http://wol.ra.phy.cam.uk/mackay/codes/data.html. 
[28] M. Luby, M.Mitzenmacher, A. Shokrollahi, and D. Spielman, "Analysis of low-density codes and improved designs using irregular graphs," Proc. 30th ACM STOC, Dallas, TX, 1998, pp. 249-258.

[29] M. Luby, M. Mitzenmacher, A. Shokrollahi, and D. Spielman, "Improved low-density parity-check codes and using irregular graphs and belief propagation," Proc. 1998 IEEE Int. Symp. on Information Theory, Cambridge, MA, Aug. 1998, p. 117.

[30] S. L. Jacoby, J. S. Kowalik, J. T. Pizzo, and W. T. Veterling, Iterative Methods for Nonlinear Optimization Problems, Prentice-Hall, Inc., Englewood Cliffs, New Jersey, 1972.

[31] W. H. Press, B. P. Flannery, S. A. Teukolsky, and W. T. Veterling, Numerical Recipes in C, Cambridge, 1988.

[32] S. B. Wicker, Error Control Systems for Digital Communications and Storage. PrenticeHall, Inc., 1995.

[33] D. M. Mandelbaum, "An adaptive Feedback Coding scheme Using Incremental Redundancy," IEEE Trans. Inform. Theory, pp. 388-389, May 1974.

[34] Y. Wang and S. Lin, "A Modified Selective-Repeat Type-II Hybrid-ARQ System and is Performance Analysis," IEEE Trans. Comm., vol. 31, pp. 593-607, May 1983.

[35] S. Lin and P.S. Yu, "Hybrid ARQ Scheme with parity retransmission for error control of satellite channels," IEEE Trans. Comm., vol. 30, pp. 1701-1719, July 1982.

[36] J. Hagenauer, "Rate-compatible punctured convolutional codes (RCPC codes) and their applications," IEEE Trans. Comm., vol. 36, pp. 389-400, 1988.

[37] Z. Lin and A. Svensson, "New rate-compatible repitition convolutional codes," IEEE Trans. Inform. Theory, vol. 46, no. 7, pp. 2651-2657, Nov. 2000. 
[38] J. B. Cain, G. C. Clark, and J. M. Geist, "Punctured convolutional codes of rate (n-1)/n and simplified maximum likelihood decoding," IEEE Trans. Inform. Theory, vol. 25, pp. 97 100, Jan. 1996.

[39] R. G. Gallager, Information Theory and Reliable Communication. New York: John Wiley and Sons, 1968.

[40] D. N. Rowitch, and L. B. Milstein, "Rate compatible punctured turbo (RCPT) codes in a hybrid FER/ARQ system," Proc. GLOBECOM, vol.4, pp. 55-59, Phoenix, AZ, Nov. 1997.

[41] P. Jung and Plechinger, "Performance of rate compatible punctured turbo-codes for mobile radio applications," Electron. Lett., vol. 33, pp. 2102-2103, Dec. 1997.

[42] J. Li and H. Imai, "Performance of hybrid-ARQ protocols with rate-compatible turbo codes," in Proc. 1997 Int. Symp. on Turbo Codes, Brest, France, Sept. 1997, pp. 188-191.

[43] D. Chase, "Code combining - a maximum-likelihood decoding approach for combining an arbitrary number of noisy packets," IEEE Trans. Comm., vol. 33, pp. 385-393, May 1985.

[44] B. A. Harvey and S. B. Wicker, "Packet combining systems based on the Viterbi decoder," IEEE Trans. Comm., vol. 42, pp. 1544-1557, Feb./Mar./Apr. 1994.

[45] R. Mantha and F. R. Kschischang, "A capacity-approaching hybrid ARQ scheme using Turbo codes," Proc. GLOBECOM, pp. 2341-2345, 1999.

[46] D. N. Rowitch and L. B. Milstein, "On the performance of hybrid FEC/ARQ systems using rate compatible punctured turbo (RCPT) codes," IEEE Trans. Comm., vol.48, No.6, pp.55-67, June, 2000.

[47] J. Ha and S. W. McLaughlin, "Analysis and design of LDPCCs over Gaussian channels with erasures," in Proc. of IEEE Intl. Symp. Inform. Theory (ISIT), Lausanne, Switzerland, 2002 , 
[48] J. Ha, and S. W. McLaughlin, "Optimal puncturing distributions for rate-compatible low-density parity-check codes," Submitted to IEEE Trans. Inform. Theory.

[49] J. Li and K. Narayanan, "Rate-Compatible Low Density Parity Check Codes for Capacity-Approaching ARQ Scheme in Packet Data Communications," in Int. Conf. on Comm., Internet, and Inf. Tech. (CIIT), Nov. 2002.

[50] $3^{\text {rd }}$ Generation Partnership Project (3GPP), "High Speed Downlink Packet Access," 3GPP TR25.885, June 2001.

[51] F. Zarkeshvari and A. H. Banihashemi, "On implementation of min-sum algorithm for decoding low-density parity-check (LDPC) codes," Proc. GLOBECOM, pp. 1349-1353, 2002. 